\title{
A Transdisciplinary Perspective of Chronic Stress in Relation to Psychopathology Throughout Lifespan Development
}

\author{
Robert-Paul Juster ${ }^{1,2 *}$, Gustav Bizik ${ }^{3}$, Martin Picard ${ }^{4}$, Genevieve Arsenault-Lapierre ${ }^{1,2,5}$, \\ Shireen Sindi ${ }^{1,2}$, Lyane Trepanier ${ }^{1,6}$, Marie-France Marin ${ }^{1,7}$, Nathalie Wan ${ }^{1}$, \\ Zoran Sekerovic ${ }^{1,8}$, Catherine Lord ${ }^{9}$, Alexandra J. Fiocco ${ }^{10}$, Pierrich Plusquellec ${ }^{1}$, \\ Bruce S. McEwen ${ }^{11}$, Sonia J. Lupien ${ }^{1,12}$
}

\author{
Affiliations: \\ ${ }^{1}$ Centre for Studies on Human Stress, Fernand-Seguin Research Centre at Louis-H. \\ Lafontaine Hospital, University of Montreal, Canada \\ ${ }^{2}$ Dept. of Neurology and Neurosurgery, McGill University, Canada \\ ${ }^{3}$ Center for Neuropsychiatric Research of Traumatic Stress, Dept. of Psychiatry, \\ Faculty of Medicine, Charles University, Czech Republic \\ ${ }^{4}$ Dept. of Kinesiology and Physical Education, McGill University, Canada \\ ${ }^{5}$ Bloomfield Center for Research in Aging, Lady Davis Institute for Medical Research, \\ Jewish General Hospital, Canada \\ ${ }^{6}$ Biomedical Sciences, University of Montreal, Canada \\ ${ }^{7}$ Dept. of Neurological Sciences, University of Montreal, Canada \\ ${ }^{8}$ Dept. of Psychology, University of Montreal, Canada \\ ${ }^{9}$ Centre de recherche de l'institut universitaire de gériatrie de Montréal, Canada \\ ${ }^{10}$ Baycrest Centre, The Kunin-Lunenfeld Applied Research Unit, Canada \\ ${ }^{11}$ Harold and Margaret Milliken Hatch Laboratory of Neuroendocrinology, Rockefeller \\ University, United States of America \\ ${ }^{12}$ Dept. of Psychiatry, University of Montreal, Canada
}

"Corresponding Author:

Robert-Paul Juster

7401 Hochelaga, Louis Riel Pavilion, Unit 226, Room RI-2678

Montreal, Quebec, Canada, H1N 3M5

Tel: (514) 251-4000, Ext. 3253

Fax: (514) 251-7968

Email: robert.juster@mail.mcgill.ca 


\title{
Transdisciplinary Allostatic Load Review
}

\begin{abstract}
The allostatic load model represents an interdisciplinary approach to comprehensively conceptualize and quantify chronic stress in relation to pathologies throughout the life cycle. This article first reviews the allostatic load model, followed by interactions among early adversity, genetics, environmental toxins, as well as distinctions among sex, gender, and sex hormones as integral antecedents of allostatic load. We next explore perspectives on severe mental illness, dementia, and caregiving as unique human models of allostatic load that merit future investigations in the field of developmental psychopathology. A complimenting transdisciplinary perspective is applied throughout, whereby we argue that the allostatic load model goes beyond traditional stress-disease theories towards the advancement of person-centered research and practice that promote not only physical health but also mental health.
\end{abstract}

Keywords: chronic stress; allostatic load; transdisciplinarity; life cycle model of stress; developmental psychopathology; multilevel analysis; glucocorticoids 


\section{Transdisciplinary Allostatic Load Review}

\section{Introduction}

Stress researchers require interdisciplinary frameworks to study the effects and consequences of chronic stress. One major obstacle in understanding and defining stress is that it is a multidimensional construct involving interactions among inputs (environmental stressors), processes (subjective psychological distress), and outputs (objective biological stress responses) (Levine, 2005; Levine \& Ursin, 1991) that are often investigated separately from each other. Over the last two decades, the concepts of allostasis (Sterling \& Eyer, 1988) and of allostatic load (McEwen \& Stellar, 1993) have served as the impetus for hundreds of publications that go far beyond the perspective that "stress" is an exclusively social, psychological, or biological construct, but rather all constructs studied together in an integrative framework (for an earlier review, see (Lupien et al., 2006).

Originating as a biopsychosocial model, allostatic load (AL) refers to the 'wear and tear' the body experiences when organisms are subjugated to chronic stress. Central to this biological damage is altered stress hormone functioning that inexorably strains interconnected biomarkers that eventually collapse like domino pieces trailing towards stress-related endpoints. The traditional operational definition of the $\mathrm{AL}$ index is the sum number of dysregulated neuroendocrine, immune, metabolic, and cardiovascular biomarkers (Seeman, Singer, Rowe, Horwitz, \& McEwen, 1997a). This aggregate or AL index has then been used to tease apart the psychosocial determinants of disordered, diseased, and deceased outcomes. An emerging body of literature clearly demonstrates that the AL index is associated with numerous physical outcomes throughout lifespan development and even psychopathological outcomes (for an earlier review, see Juster, McEwen, \& Lupien, 2010).

With the aim of refining the AL model further in application to psychopathological phenomena throughout the life cycle, we offer the following review of the causes and effects of 


\section{Transdisciplinary Allostatic Load Review}

AL. The first part of this article will review literature on powerful antecedents of AL: (a) early adversity that predisposes individuals to life-long disease susceptibilities; (b) genetic and epigenetic factors; (c) mediation or moderation by environmental toxins; as well as (d) interactions among biological sex, socio-cultural gender, and sex hormones. We follow secondly with an exploration of pathophysiological manifestations that could be used as human models of AL: (e) living with schizophrenia or bipolar disorder; (f) the dementia spectrum; and finally (g) the experience of caregiving. Our hope is to transcend the boundaries of existing theory, research, and practice by proposing that the AL model can evolve into a transdisciplinary framework that ushers a holistic approach to understand and help distressed individuals. Sharing key similarities with developmental psychopathological models, a transdisciplinary AL model likewise would seek to address the causes, mechanisms, and heterogeniety manifested among diverse stressrelated disease trajectories.

Transdisciplinarity is an approach to scientific inquiry aimed at solving complex research questions by transcending traditional disciplinary boundaries (Kessel \& Rosenfield, 2008; Rosenfield, 1992). The complexities inherent among numerous interacting factors that contribute to an individual's experience of health or disease necessarily calls for transdisciplinary models that do more than integrate knowledge (Albrecht, Freeman, \& Higginbotham, 1998; Kessel, Rosenfield, \& Anderson, 2008). In transdisciplinary health models, our concepts of health can evolve beyond the absence of disease, and even beyond the sum of biopsychosocial parts towards more holistic ones.

In this view, health is an emergent property of individuals taken as a whole (Picard, Sabiston, \& McNamara, 2011) (see Figure 1). Indeed, all factors discussed throughout this review interact in unique ways to influence one's risk of or protection against the noxious effects of chronic stress. In this spirit, we encourage the reader to contextualize the presented information 


\section{Transdisciplinary Allostatic Load Review}

within their respective area(s) of expertise in partnership with others and especially vis-à-vis complementary perspectives espoused in the field of developmental psycholopathology.

Briefly, the defining features of developmental psychopathology are (1) understanding causal processes, (2) elucidating developmental mechanisms, and (3) emphasis on diverse conceptualizations of continuities and discontinuities between spectrums of normality and abnormality in mental health (Rutter \& Sroufe, 2000). Such integrative perspectives focus on how multiple causes and effects contribute to probabilistic rather than deterministic pathways leading to diverse outcomes (Cichetti \& Toth, 2009). In particular, 'developmental analyses' highlight the dynamic nature of individual differences in vulnerabilities and resiliencies shaped by interacting factors studied from the micro to maco level (Cichetti \& Toth, 2009). The AL model shares much in this diverse spirit of analysis bridging numerous disciplines together. Yet, empirical substantiations of AL theory remain largely based on population-level analyses.

Specifically, the AL model has been primarily applied to detect physical morbidities and all-cause mortality in epidemiological analyses. While promising, it has been challenging to propose applications towards more individual-level analyses pertinent to understanding complex psychopathological phenomena. Notable exceptions have emerged; however, there remains some resistance to extensively investigate $\mathrm{AL}$ in humans in the neurosciences, psychiatry, and psychology. To address this, our principal objectives in this article are twofold: 1) to review the AL model from a developmental psychopathology perspective in relation to current and future research areas; and 2) to outline transdisciplinary interactions between key AL factors and psychopathological conditions. We begin with a brief introduction of key terms and concepts.

Stress and the neurobiology of stress responses 


\section{Transdisciplinary Allostatic Load Review}

Stress is here defined as a real or interpreted threat to an individual's physiological and psychological integrity that results in adaptive biological and behavioral responses (McEwen \& Seeman, 1999). Situations that are novel, unpredictable, threatening to the ego/self, and/or uncontrollable additively contribute to physiological stress responses (Dickerson \& Kemeny, 2004; Lupien et al., 2006; Mason, 1968). Under these circumstances, stress responses are activated by the autonomic nervous system and neuroendocrine system to mobilize energy necessary to adapt to the demands of the situation (McEwen, 1998b; Sapolsky, Romero, \& Munck, 2000).

At first, perceived and interpreted threats trigger the sympathetic-adrenal-medullary (SAM) axis to release catecholamines (e.g., adrenalin) within seconds from the adrenal medulla surrounding the kidneys. Secondly, the hypothalamic-pituitary-adrenal (HPA) axis is activated within minutes to produce glucocorticoids. The hypothalamus activates the HPA-axis by stimulating corticotrophin-releasing factor (CRF). CRF then travels through a portal system linking the hypothalamus to the pituitary, where it signals the secretion of adrenocorticotrophin hormone $(\mathrm{ACTH})$ from the anterior pituitary rich with blood capillaries. ACTH then travels viscerally to the adrenal cortex surrounding the kidneys where it binds to receptor tissue and precipitates cellular activities in the zona fasticullata region of the adrenal cortex to produce glucocorticoids (GCs) such as cortisol in humans (Sapolsky et al., 2000). The resulting surges in catecholamines and GCs alter homeostasis by appropriating biological functions that permit fightor-flight responses.

The brain's ultimate role during stress responses is to detect threat and adapt. In addition to the pituitary and hypothalamic activities, there are three major brain structures involved in the regulation of stress responses: (1) the hippocampus linked to memory and cognition, in addition to being implicated in negative feedback regulation of the HPA-axis; (2) the amygdala responsible 


\section{Transdisciplinary Allostatic Load Review}

for fear conditioning and emotional processing with outputs to autonomic and neuroendocrine regulatory systems, and; (3) the prefrontal cortex involved in cognition and coping strategies and exerting top-down control over subcortical structures (Gray \& Bingaman, 1996; McEwen, 2004; McEwen, Weiss, \& Schwartz, 1968; Reul \& de Kloet, 1985; Sanchez, Young, Plotsky, \& Insel, 2000; Thayer \& Lane, 2009). In concert with the autonomic nervous system, short-term adaptations of organisms during stress responses are dynamic biological processes that ensure survival.

\section{Concept of allostasis}

SAM- and HPA-axis stress responses are examples of allostasis, defined as adaptive biological processes that preserve "stability through change" (Sterling \& Eyer, 1988). This concept was coined by an epidemiologist and a neurobiologist to describe how the body maintains homeostasis by allocating metabolic energy to cope with the demands of the environment (Sterling, 2004). In contrast to homeostasis that focuses on static set-points (e.g., Ph levels, core body temperature), the concept of allostasis focuses on how continuous re-evaluation and readjustments create new set-points that maximize the organism's resources (e.g., increased cardiac output when running). Likewise, homeostatic mechanisms cannot adequately account for how the brain appropriates itself beyond rigidly enforced biological mechanisms. For instance, when increased needs create an error signal, negative feedback mechanisms may try to correct the error and cause more error (e.g., rapidly rising causing head-rush and dizziness). Allostasis can drive behaviors explaining how anticipation of physiological change can rectify these errors (e.g., getting up slowly to prevent dizziness) and in this way, considers an unusual parameter value not as a failure to defend a set-point, but rather as a response to some prediction (Sterling, 2004; 


\section{Transdisciplinary Allostatic Load Review}

(Goldstein \& Kopin, 2007). This perspective allows for a greater understanding of the relation between physiological needs, behavior, and pathology (Sterling \& Eyer, 1988).

Homeostatic models generally define health as an internal milieu whereby all physiological parameters have relatively normal values. Disease, on the other hand, occurs when normal ranges fall into abnormal but pharmaceutically correctable ranges. In this view, Sterling and Eyer (1988) argued that the medical model is in danger of iatrogenesis, that is, the deleterious effects that certain treatments can inadvertently induce. Iatrogenic effects can arise when restoring one parameter to a normal range causes other parameters to become "inappropriately" high or low and cause altogether separate health issues (e.g., hypertension medications can negatively alter interconnected biological systems). In contrast, the allostatic model defines health as a state of responsiveness. A parameter with values outside the normal range is not necessarily considered inappropriate as each parameter is controlled by a multitude of mutually reinforcing signals (Sterling, 2004).

In summary, allostasis fundamentally differs from homeostasis because of its emphasis on dynamic rather than static biological set-points, considerations of the brain's role in feedback regulation (for a review of allostasis and the brain, see (Ganzel, Morris, \& Wethington, 2010), as well as its view of health as a whole-body adaptation to contexts (Schulkin, 2003b). According to allostatic theory, pathology itself is a form of adaptation where, for instance, an individual develops hypertension in order to conciliate environmental demands, even if these processes are pathophysiological. Health in allostatic theory is therefore defined as optimal predictive fluctuation, whereby shifts in the probability of demand should shift the response, and when the prediction reverses, so should the response (Sterling, 2004). In this view, the allostasis concept is consistent with a transdisciplinary definition of health and disease emphasizing holistic relationships among individuals and environments. It is, however, when these adaptive systems 


\section{Transdisciplinary Allostatic Load Review}

become chronically dysregulated that maladaptive physiological consequences emerge. The concept of allostasis therefore represents a multi-faceted mechanism of regulation applicable to psychopathological as well as pathophysiological research by uniting mind and body in explanations of dynamic stability that can go astray.

\section{The allostatic load model}

McEwen \& Stellar (1993) expanded upon allostasis by proposing that AL is the price that the body incurs when bombarded by stressful situations and prolonged stress responses. The principal points of the AL model are twofold: (1) AL involves multiple interconnected biomarkers that can falter primarily as a function of stress hormone dysregulations, and that (2) the health sciences should not wait idly by for individuals to reach clinical levels on these biomarkers but should instead identify sub-clinical states before they lead to pathology.

To substantiate these arguments, the AL model synthesizes a rich, interdisciplinary literature on stress and disease by elucidating how numerous factors converge to predispose individuals to stress-related pathologies over the life cycle (McEwen, 2000a). This endeavor necessitates fusion of multiple knowledge bases, as coordination of allostasis depends on the brain's evaluation of threat and execution of physiological responses (Herman, Ostrander, Mueller, \& Figueiredo, 2005; McEwen, 2007b, 2008). This coordination is fundamentally shaped by an individual's constitution (genetics, development), history (trauma/abuse, major life events, early adversity), and behaviors (coping and health habits) (McEwen, 1998a). Akin to developmental psychopathological emphasis on how experiences can alter developmental pathways, understanding how these diverse factors are integrated at the individual level to yield altered expressions of normative functioning can provide innovative ways of treating ill individuals and fostering positive health. 


\section{Transdisciplinary Allostatic Load Review}

In contrast to healthy allostatic responses whereby individuals react and recover to stressors effectively, allostatic states (Koob \& Le Moal, 2001) can occur when chronic dysregulation of regulatory systems lead to pathophysiological operating ranges. There are four postulated physiological profiles that represent allostatic states that contribute to AL: (1) repeatedly activated responses (2) non-habituating responses (3) prolonged responses, (4) and inadequate responses (McEwen, 1998a) (see Figure 2). Detecting these different allostatic states is possible by investigating biomarker(s) dynamics in transdisciplinary manner vis-à-vis health outcomes. Because allostatic states prompt compensatory remediation among numerous biomarkers, each of which is shaped by numerous factors, delineating time-courses of dysregulation in AL involves the challenge of determining which players falter first in the cascade (McEwen, 2008).

Multiple biomarkers are hypothesized to function as part of a non-linear network that contributes to a tripartite sequence leading to AL (McEwen, 1998a). At first, over-activation of primary mediators such as stress hormones and pro- and anti-inflammatory cytokines exact primary effects on cellular functions such as enzyme, receptor, ion channel, and genomic activities. Synergistic effects of several primary mediators then leads to tissue and organ specific changes in several systems. Subsidiary systems in turn recalibrate their own activities to compensate for the over and/or under production of primary mediators and effects.

This leads to secondary outcomes, whereby metabolic, cardiovascular, immune, and reproductive biomarkers become imbalanced. Multiple pathways interact in a non-linear fashion whereby oscillations in biomarker levels drive compensatory recalibrations of other intertwined biomarkers (see Figure 3). For example, inflammatory cytokine production is negatively regulated via anti-inflammatory cytokines as well as via parasympathetic and GC pathways, whereas sympathetic activity increases inflammatory cytokine production and parasympathetic 


\section{Transdisciplinary Allostatic Load Review}

activity restrains sympathetic activity (McEwen, 2006a). The synergistic effects of multiple primary mediators and primary effects exerting themselves with secondary outcomes progressively strains the organism until a breaking-point. AL becomes overloading when tertiary outcomes emerge with the manifestation of clinical endpoints such as cardiovascular disease and mortality.

In an evolutionary biological perspective, allostatic overload (McEwen \& Wingfield, 2003) occurs in two variants. First, type I allostatic overload occurs when energy demands exceed energy income, representing a negative energy balance. For example, lack of food because of habitat changes results in emergency life sustaining responses that pit the organism into a survival mode whereby energy stores are uncompromisingly mobilized from stores as GCs take charge (Goymann \& Wingfield, 2004; Korte, Koolhaas, Wingfield, \& McEwen, 2005). Second, type 2 allostatic overload occurs if energy demands are not exceeded and the organism continues to take in or store as much or even more energy than it needs, representing a positive energy balance. For example, today's industrialized agricultural products allow for massive food supplies that contribute to troubling obesity and metabolic problems (McEwen \& Wingfield, 2003). Transcending theory, AL and allostatic overload have been the subject of a growing empirical literature that holds as central tenants the advantages of assessing multiple biomarkers, particularly primary mediators (McEwen, 2003b), at sub-clinical levels as a function of different contexts (e.g., socio-economic status, stressful life events, toxic workplaces).

\section{Measuring allostatic load}

Work by Teressa Seeman and colleauges has been instrumental in developing an AL index that encompasses primary mediators and secondary outcomes to predict tertiary outcomes. The traditional count-based AL index was based on analyses from the MacArthur Studies of 


\section{Transdisciplinary Allostatic Load Review}

Successful Aging, a cohort of high-functioning American older adults ages 70 to 79 followed longitudinally. The original AL index included 10 biomarkers from neuroendocrine (cortisol, adrenalin, noradrenalin, and dehydroepiandrosterone-sulphate (DHEA-S)), metabolic (total cholesterol to high density lipoprotein- (HDL) cholesterol, HDL-cholesterol, glycosylated hemoglobin $\left(\mathrm{Hb}_{\mathrm{Alc}}\right)$, and waist-to-hip-ratio), and cardiovascular (systolic and diastolic blood pressure) systems (Seeman et al., 1997a). Once all biomarker values were obtained from the sample, participants' values falling within the highest-risk with respect to the sample's biomarker distributions. This has traditionally been the $75^{\text {th }}$ percentile for biomarkers whereby high levels denote disease vulnerabilities (e.g., glucose, LDL-cholesterol), while the $25^{\text {th }}$ percentile is for biomarkers whereby low levels are unhealthy (e.g., DHEA, HDL-cholesterol). The AL index is then calculated by adding together the number of biomarkers that reach these cut-offs. Using this multi-systemic index that has subsequently included diverse biomarkers (see Table 1) and alternative calculations, numerous studies have assessed the relationship between antecedents of $\mathrm{AL}$ and the functional significance of high AL.

By applying this $\mathrm{AL}$ index in analyses of the MacArthur cohort, it was shown that increased AL levels are related to cognitive and physical impairments at baseline and associated declines over three years, as well as an increased risk of having cardiovascular disease (CVD) (Seeman, Singer, Rowq, Horwitz, \& McEwen, 1997). At 7-year follow-up, those with the highest AL levels were at greatest risk of cognitive and physical decline as well as risk of CVD and even all-cause mortality. Note that while differences were found when using neuroendocrine (stress hormones and DHEA-S) versus metabolic syndrome biomarkers (glyco-lipids, blood pressure, adiposity), the multi-systemic AL index was better than using only single biomarkers or the aforementioned clusters to predict outcomes (Seeman, McEwen, Rowe, \& Singer, 2001). In a similar cross-sectional analysis, it was shown that primary mediators contribute independently of 


\section{Transdisciplinary Allostatic Load Review}

cardiovascular and metabolic risk factors in predicting cognitive and physical functioning declines (Karlamangla, Singer, McEwen, Rowe, \& Seeman, 2002). Fortunately, even in old age and regardless of sex, decrements in AL over 2 and 4 years diminishes one's risk of dying (Karlamangla, Singer, \& Seeman, 2006).

These results were cross-culturally complemented using the Social Environment and Biomarkers Study of Aging with Taiwanese aged 54 to 71 . Consistent with the MacArthur cohort, increased AL was associated with increased 3-year mortality risk (Goldman et al., 2006b). Seplaki and collaborators have contributed several important methodological studies using the Taiwanese cohort, showing that increased AL is related to poorer health status (self-rated health, activities of daily living, mobility, temporal orientation), depressive symptoms, and cognitive impairments (Seplaki, Goldman, Glei, \& Weinstein, 2005; Seplaki, Goldman, Weinstein, \& Lin, 2004, 2006). Furthermore, 3-year mortality risk, mobility limitations, cognitive decline, and depressive symptomatology were predicted by higher AL, but driven differentially by traditional clinical riskfactors vs. neuroendoimmunological mediators (Goldman, Turra, Glei, Lin, \& Weinstein, 2006a).

Seplaki and Goldman's analyses are the first to link geriatric depression to AL. About 8$20 \%$ of community-dwelling older adults will experience depression (Brundtland, 2001), which is currently the second leading cause of invalidity in the Western world after cardiovascular disease (WHO, 2001) and will further effect an estimated 1.2 to 2 billion people over the ages of 60 from 2025 to 2050 (WHO, 2002). Adverse psychosocial factors throughout life lead to higher AL levels, and by implication, to greater risk of psychopathologies as a whole. Indeed, individuals with increased perceived stress are more likely to have increased AL levels (Glei, Goldman, Chuang, \& Weinstein, 2007; Goldman, Glei, Seplaki, Lui, \& Weinstein, 2005) and risk of developing physical and psychiatric symptoms. It is important to note that such disease manifestations are rooted in past stressful experiences such as during working years. 


\section{Transdisciplinary Allostatic Load Review}

As one of the most noxious stressors adults can experience before retirement and transitions into older age, job insecurities and adverse working conditions powerfully effect disease trajectories (Taylor, Repetti, \& Seeman, 1997) and have been the subject of numerous empirical AL studies (Bellingrath, Weigl, \& Kudielka, 2009; Fischer et al., 2009; Johansson, Huang, \& Lindfors, 2007; Juster et al., In Press, In Press; Kinnunen, Kaprio, \& Pulkkinen, 2005; Langelaan, Bakker, Schaufeli, van Rhenen, \& van Doornen, 2007; Li et al., 2007; Schnorpfeil et al., 2003; Sun, Wang, Zhang, \& Li, 2007; von Thiele, Lindfors, \& Lundberg, 2006). Taken together, work-related factors (e.g., job demands, limited decision making, effort-reward imbalance, burnout symptoms) exert an adverse effect on AL levels. These workplace studies have turned the focus onto the psychosocial antecedents of AL at younger ages; however, due to their cross-sectional design, can give only limited prospective indications of how the AL index might be associated with disease outcomes in later life.

Decomposing the biopsychosocial factors that contribute to specific disease trajectories is the next step in substantiating the prospective utility of the AL model in detecting problems of the mind and body alike. Moreover, AL indices in conjunction to triangulation of methods may represent an increasingly accurate means of evaluating an individual's global health (Picard et al., 2011). This represents a holistic state emerging from the integration of multiple life domains that ultimately determine one's resilience, longevity, and life satisfaction. One must bear in mind that AL levels steadily increase with age (Juster et al., 2010) and because of this, it is increasingly important to assess AL at earlier ages, particularly during major developmental stages that shape our neurocognitive systems and patterns of responding to life's stressors (Cicchetti, 2002). Of particular interest are the "biological signatures" (Gruenewald, Seeman, Ryff, Karlamangla, \& Singer, 2006) that might characterize unique psychopathological manifestations. Such biological signatures might give indications of an individual's probability of developing particular ailments 


\section{Transdisciplinary Allostatic Load Review}

in the future. In this vein, we turn now to literature on the development of $\mathrm{AL}$ and psychopathology as they begin in early life.

\section{Biological Embedding in Early Life}

Early life adversities can unfavorably alter allostatic mechanisms (McEwen, 2003a). Hertzman introduced the term biological embedding to characterize the processes whereby cumulative disadvantages and stressful and/or traumatic experiences during youth lead to calibrations of the individual's life-long response patterns to stressors that predispose them to morbidities and premature mortality (Hertzman, 1999; Hertzman \& Wiens, 1996). Based on groundbreaking studies of maternal handling in rodents (Meaney, Aitken, van Berkel, Bhatnagar, \& Sapolsky, 1988), exemplary parenting in rhesus monkeys (Suomi, Delizio, \& Harlow, 1976), and social dominance hierarchies in free-ranging baboons (Sapolsky, 2005), Hertzman further argues that biological embedding occurring across the animal kingdom parallels socio-economic gradients in humans.

\section{Early adversity and low socio-economic status}

Before birth and thereafter, a child's development is heavily influenced by factors related to socioeconomic status (SES) and linked to detectable AL levels in preadolescence. Gary Evan's pioneering work has demonstrated that as early as age nine, increased AL indices are associated with greater frequency of factors more prevalent in low SES environments (e.g., crowding, noise, violence, single parenthood) (Evans, 2003). By age 13, early adversities in combination with low maternal responsiveness exacerbate AL levels further (Evans, Kim, Ting, Tesher, \& Shannis, 2007). Ultimately, exposure to chronic stress during childhood exacts life-long health 


\section{Transdisciplinary Allostatic Load Review}

consequences and propels youths to view the world as more threatening and challenging (McEwen, 2003a).

At the core of adverse biological embedding in disadvantaged youths is cortisol's ability to modulate cognitive processes that increase vigilance (Buchanan \& Lovallo, 2001; Lupien et al., 2006). Consistently, we reported that low SES school-aged children have increased cortisol levels in the absence of attention and memory impairments (Lupien, King, Meaney, \& McEwen, 2001). Lower SES children also report feeling that things in life are more impossible in an emotional plausibility task used in a separate study (Lupien et al., 2005), perhaps explaining how individuals born into low SES often remain less educated or wealthy. This intriguing result does not underlie a cognitive deficit per se, but nevertheless represents a cognitive divergence that might impact how low SES children approach and/or avoid certain life situations and opportunities. By age 17, adolescents that have lived in chronic poverty not only have increased AL levels but also working memory impairments (Evans \& Schamberg, 2009). Another study found that $9^{\text {th }}$ to $12^{\text {th }}$ grade adolescents coming from families with lower education (Goodman, McEwen, Huang, Dolan, \& Adler, 2005) also had higher AL indices, which suggests that SES-related vulnerabilities are indeed partly inter-generationally passed on.

On the other hand, childhood SES does not determine health by itself, as life trajectories in social mobility, social relationships, and parental bonding interactively predict AL levels in adulthood (Singer, Friedman, Seeman, Fava, \& Ryff, 2005; Singer \& Ryff, 1999). While resiliency factors like good parenting behavior can help buffer the effects of economic hardships (Houston \& Vavak, 1991), the absence of facilitation when facing early stressful situations only contributes further to earlier signs of AL (Hamil-Luker \& O'Rand, 2007; Repetti, Taylor, \& Seeman, 2002). Low SES, in addition to factors such as dysfunctional familial dynamics and abuses, will shape a child's ability to discriminate threatening from non-threatening information 


\section{Transdisciplinary Allostatic Load Review}

that consequently distinguishes whether cognitive processes will lead to hardiness against or heightened sensitivity to stressors (Lupien et al., 2006).

The 'risky' family, inter-parental conflict, child abuse, and cognitive adaptations

The risky family model describes family systems marked by chronic interpersonal dysfunction (Repetti et al., 2002). Children growing up in risky families with high conflict and aggression as well as cold, unsupportive, and/or neglectful parenting are more likely to experience marked impairments in AL biomarker functioning, social competencies, impaired growth, and sexual development that can precipitate disease outcomes in adulthood (Repetti et al., 2002; Taylor et al., 1997). Even when family dysfunction leads to dissolution, symptoms of high chronic stress exposure can be pervasive for offspring. For example, parental divorce can represent a major stressful life event for some youths that can lead to geographic relocations (Braver, Ellman, \& Fabricius, 2003), altered circadian HPA-axis functioning (Kelly, Young, Sweeting, Fischer, \& West, 2008), increased AL levels (Evans, 2003; Evans et al., 2007), and increased risk of depressive symptoms (Chase-Lansdale, Cherlin, \& Kiernan, 1995; Gore, Aseltine, \& Colton, 1992; Huurre, Junkkari, \& Aro, 2006). Essentially, youths that must attend to constant barrages of stressful circumstances in the absence of coping resources are more vulnerable to numerous stressrelated diseases throughout their lifetimes.

Unfortunately in some cases, risky family climates sometimes transform into direct verbal, emotional, physical, and sexual abuse, and/or neglect of youths that can further contribute to deregulations in HPA-axis function and increased AL (Cicchetti \& Rogosch, 2001; McEwen, 2000b). Furthermore, children with blunted morning cortisol levels who have been emotionally maltreated and/or neglected, but not physically and/or sexually abused make more false recognition memory errors (Cicchetti, Rogosch, Howe, \& Toth, 2010b), as do adults who were 


\section{Transdisciplinary Allostatic Load Review}

similarly maltreated when young (for a review see Howe, Toth, \& Cicchetti, 2006). That specific forms early life traumas have differential impacts on HPA-axis functioning represents an important development towards the identification of neurobiological signatures linked to psychopathological vulnerabilities (Cicchetti, Rogosch, Gunnar, \& Toth, 2010a; Heim, Newport, Mletzko, Miller, \& Nemeroff, 2008; Heim, Plotsky, \& Nemeroff, 2004). A review by Bauer and Boyce (2004) suggests that child abuse and intra-familial dysfunctions are also associated with numerous tertiary outcomes of AL. This literature collectively suggests that early adversities and familial dysfunctions contribute to biological embedding that alters how youths perceive and cognitively interpret past, present, and future stressors.

It is important to highlight how physiological dysregulations are conceptually linked to eventual cognitive deficits, but developing cognitive capacities in early life can also function as perpetuators of vicious circles that amplify the effects of chronic stress in promotion of impending psychopathology. Such cognitive processes have been touched upon by the concept of preservative cognitive appraisals (Brosschot, Gerin, \& Thayer, 2006; Brosschot, Pieper, \& Thayer, 2005; Brosschot \& Thayer, 2003) defined as over-activated cognitive representations of stressors (e.g., excessive worrying, rumination, and anticipatory stress) that prolong stress responses and promote allostatic states and AL. Just as individual differences in exposure to early adversities can modulate cognitive schemas that impel preservative cognitions, so too can the reversal of these propensities by psychotherapeutic means and broader social policies.

As prevention is not always possible and children must often endure face familial challenges until the end of adolescence and thereafter, the reversal of AL and psychopathological sequelae might be ideally implemented through early interventions (Shonkoff, Boyce, \& McEwen, 2009). Community outreach programs and psychotherapies that aim to provide family education, moral, and social support to both parents and youths can lessen the impact of early childhood 


\section{Transdisciplinary Allostatic Load Review}

adversity (Bauer \& Boyce, 2004) and effectively reduce, for instance, delinquency rates (Gomby, Larner, Stevenson, Lewit, \& Behrman, 1995). Notwithstanding, public education is crucial for garnering the support and the resources necessary to sustain and refine these programs (Bauer \& Boyce, 2004). Recognizing that the problem is transdisciplinary in nature is essential and has the potential to contribute to the success of these programs (Kessel et al., 2008). It is during these formative years that positive factors can counteract the biological embedding impelled by negative circumstances. As the following will illustrate, buffering the effects of AL during critical periods can facilitate resilience.

\section{Life cycle model of stress}

The timing and duration at which major stressors and/or traumas are experienced profoundly effects the neurological development and the vulnerabilities or resistance individuals have towards experiencing specific psychopathologies (Lupien, McEwen, Gunnar, \& Heim, 2009). With regards to brain regions central to HPA-axis regulation in humans, the hippocampus attains complete maturation first at age two, the prefrontal cortex (PFC) next between ages eight to fourteen, and finally the amygdala continues to grow slowly until the late 20s (Giedd et al., 1996; Yakovlev, 1967). The life cycle model of stress proposed by Lupien, McEwen, Gunnar, and Heim (2009) states that exposure to adversity and chronic stress during key critical periods can impair the development of these brain regions, particularly those that have not fully matured. In this manner, smaller regional volumes in conjunction to biological signatures (e.g., hypercortisolism vs. hypocortisolism) can be used to predict differential risk-profiles for specific psychopathologies (e.g., depression vs. post traumatic stress disorder) in adulthood as well as inform when certain traumas might have occurred (Lupien et al., 2009). 


\section{Transdisciplinary Allostatic Load Review}

The growth and decline of these brain regions depends on the developmental phases at which the stressors are experienced, which leads to differential effects on HPA-axis functioning: (a) prenatal stress leads to programming effects, (b) postnatal stress leads to differentiation effects, (c) stress in adolescence leads to potentiation/incubation effects, and (d) stress in adulthood and aging culminate with maintenance/manifestation effects (Figure 4). For example, if an individual experiences major stressors in early adulthood, there is less chance that this will structurally affect their already fully matured hippocampus or frontal lobes, but could instead lead to hypertrophy of the amygdala as this region is still maturing. This may then lead to maintenance/manifestation effects and psychopathologies later on in adulthood.

This model brings together the hithero opposing neurotoxicity hypothesis (Sapolsky, Krey, \& McEwen, 1986) and vulnerability hypothesis (Gilbertson et al., 2002): the former argues that prolonged exposure to GCs causes neurological damage, while the latter argues that neurological impairments are the cause of abnormal GCs dysregulations, and as such, represent a risk-factor for the development of psychopathologies rooted in genetics and early life events (Lupien et al., 2009).

In this vein, early adversities are associated with neurological impairments at the level of the hippocampus, amygdala, and PFC that render individuals more vulnerable to developing numerous psychopathologies (McEwen \& Gianaros, 2010; Teicher et al., 2003). Animal studies reveal that both prenatal and postnatal exposures biologically embed the HPA-axis by shaping cerebral regions like the hippocampus (Champagne \& Meaney, 2001; McEwen, 2003a) that leads to programming and differentiation effects. In humans, children who have experienced severe abuse show decreased overall brain volumes and are more susceptible to earlier onsets of posttraumatic stress disorder (PTSD) with longer disease durations (De Bellis et al., 1999). 


\section{Transdisciplinary Allostatic Load Review}

In contrast to adult studies that consistently report smaller hippocampal volume (HV) in PTSD patients (Bremner et al., 1997; Karl et al., 2006), most child PTSD studies find no evidence for structural hippocampal aberrations (Carrion et al., 2001; De Bellis, Hall, Boring, Frustaci, \& Moritz, 2001; De Bellis et al., 1999). A recent meta-analysis confirmed that children with PTSD do not necessarily experience smaller $\mathrm{HV}$, although adults with histories of childhood maltreatment often do (Woon \& Hedges, 2008). For instance, individuals with a history of childhood sexual abuse have decreased HVs (Vythilingam et al., 2002) as well as neuropsychological deficits (Bremner, Vermetten, Afzal, \& Vythilingam, 2004; Navalta, Polcari, Webster, Boghossian, \& Teicher, 2006). A recent study uncovered a sex disparity in relation to these early exposures, whereby low birth weight predicted smaller $\mathrm{HV}$ in adulthood only in women reporting low parental care but not men (Buss et al., 2007). These findings suggest that the effects of the prenatal environment combined with childhood maltreatment may not appear until adulthood or late adolescence; however, it is possible that these children had smaller HV to begin with due to prenatal factors.

One's risk of developing major depression is also modulated by early adversity. Individuals with major depression often have heightened basal cortisol levels (Burke, Davis, Otte, \& Mohr, 2005; Porter \& Gallagher, 2006), hippocampal-dependent declarative memory impairments (Burt, Zembar, \& Niederehe, 1995; Fossati et al., 2004) and encoding deficits (Bremner, Vythilingam, Vermetten, Vaccarino, \& Charney, 2004). Additionally, they have decreased HV (Campbell, Marriott, Nahmias, \& MacQueen, 2004; Videbech \& Ravnkilde, 2004) that actually predicts the frequency and duration of subsequent major depressive episodes (Campbell et al., 2004; MacQueen et al., 2003; Sheline, Sanghavi, Mintun, \& Gado, 1999; Videbech \& Ravnkilde, 2004). When investigating the association between major depression and reduced $\mathrm{HV}$, it is important to take into account whether participants have a history of abuse or 


\section{Transdisciplinary Allostatic Load Review}

maltreatment during childhood (Heim \& Nemeroff, 2001). For example, women with a history of childhood sexual or physical abuse are more likely to have hyperactive HPA-axis functioning (Heim et al., 2000) as well as smaller HV, whereas women without a history of abuse often show similar HV to healthy controls (Vythilingam et al., 2002). That differences in history of abuse lead to variant neuroendocrine functioning suggest that the nature and especially the timing of stressors and/or traumas lead to alternative forms of biological embedding with predispositions to specific diseases (Cicchetti et al., 2010a).

Consistent with this idea, patients with PTSD often show decreased levels of basal cortisol (Yehuda, Golier, \& Kaufman, 2005) instead of the hypercortisolemic profile often found in depression. These alterations in HPA-axis functioning may commence in childhood following abuse or neglect, subsequently increasing the risk for PTSD in adulthood. Indeed, combat veterans with PTSD are more likely to have histories of childhood abuse in comparison to veterans who do not develop PTSD (Bremner, Southwick, Johnson, Yehuda, \& Charney, 1993). While adult PTSD patients have decreased HV (Karl et al., 2006; Smith, 2005), hippocampal atrophy may be either a consequence of PTSD, or instead a form of vulnerability towards developing PTSD (Gilbertson et al., 2002; Lupien et al., 2007). Supporting this is a classic monozygotic-twin study, whereby combat veterans who went to war and developed PTSD as well as their twins who did not go to combat both had smaller HV in comparison to twin-pairs that did not develop PTSD (Gilbertson et al., 2002). By contrast, when HV was measured soon after exposure to trauma and before the development of PTSD symptoms, no differences were found between those that later developed PTSD and those that did not (Bonne et al., 2001). This chicken-egg phenomenon will only be better understood with prospective life-spanning studies.

Dysregulations of HPA-axis activity in psychiatric conditions such as depression and PTSD may alter other physiological biomarkers and consequently increase AL (McEwen \& 


\section{Transdisciplinary Allostatic Load Review}

Gianaros, 2010). In the only study of its kind in the AL literature, it was found that mothers of childhood cancer survivors who experienced increased distress and PTSD symptoms also had increased AL levels driven in part by lower cortisol levels (Glover, Stuber, \& Poland, 2006) in addition to smaller right HVs (Glover, Garcia-Arcena, \& Mohlman, 2008) when compared to control mothers. These analyses utilized both high and low ends of cortisol variation since hypocortisolism characterizes certain diseases (Fries, Hellhammer, \& Hellhammer, 2005). Indeed, hypocortisolism has been empirically substantiated vis-à-vis AL and symptoms of chronic fatigue syndrome (Goertzel et al., 2006; Hellhammer, Schlotz, Stone, Pirke, \& Hellhammer, 2004; Maloney, Boneva, Nater, \& Reeves, 2009; Maloney et al., 2006; Smith, Maloney, Falkenberg, Dimulescu, \& Rajeevan, 2009), post-traumatic stress disorder (Glover, 2006; Glover et al., 2008, Glover et al. 2006), and burnout (Juster et al., 2010). More research is needed to disentangle specific pathways emergent from earlier life experiences that shape the neurobiological substrates associated with these differential biological signatures (e.g., hypo- versus hyper-cortisolism). In addition to furthering our understanding of causal mechanisms, such knowledge would guide diagnostic approaches and improve the efficacy of early interventions.

\section{Conclusion and future directions}

Early adversities such as low SES (for a review, see (Dowd, Simanek, \& Aiello, 2009), living in risky family environments, suffering neglect and/or abuses, can all lead to biological embedding that increases AL and lifelong psychopathological susceptibilities. We believe that these deleterious effects on the hippocampus, amygdala, PFC, and overall brain functioning depend on the developmental timing and duration of elevated GC exposure (Lupien et al., 2009). The balance between adaptive neuroplasticity and neurodegeneration suggests an important dynamic, whereby marked brain changes occur even after maturation and persist throughout the 


\section{Transdisciplinary Allostatic Load Review}

life cycle. Therefore, dynamic parameters of brain changes in childhood and adolescent neurodevelopment, as well as later life alterations should be taken into account when analyzing processes mediating the consequences of stress exposure on the brain. An important additional element of this puzzle that has received limited substantiation in the AL literature are the genetic vulnerabilities we inherit. Indeed, genetic and epigenetic interactions can function as strings of fate that can protract biological embedding effects throughout a lifetime. Yet, genetic vulnerabilities and early adversity can also be reversed in certain enriching contexts, underlining a probabilistic rather than deterministic perspective.

For decades, diathesis-stress models have dominated theoretical discourse on how genetic predispositions to diseases can be amplified when stressors reach an intolerable threshold. This concept has many merits; however, it limits science's ability to go beyond hypotheses that fixate on vulnerability and which often cannot account for how diatheses sometimes reverse into a resilient trait. In this spirit, the Swedes have an expression called maskrosbarn (dandelion child) and a neoglism called orkidebarn (orchid child) that offers an empowering, alternative perspective to append into diathesis-stress models (Boyce \& Ellis, 2005). Quoting David Dobbs; “most of us have genes that make us as hardy as dandelions: able to take root and survive almost anywhere. A few of us, however, are more like the orchid: fragile and fickle, but capable of blooming spectacularly if given greenhouse care” (Dobbs, 2009, p. 60).

This perspective states that inherited genotypes with potentially negative consequences can be modulated to generate a multitude of potentially positive phenotypes. In terms of evolution, natural selection has permitted certain "bad" genes to remain in the gene pool if, under certain circumstances, they could enhance fitness and survival (Dobbs, 2009). With this in mind, it is important to recognize that diverse interactions among nature (genes) and nurture (environment) are unremittingly co-dependent. 


\section{Transdisciplinary Allostatic Load Review}

\section{Human Genetics: A Common Missing Piece of the Model}

A comprehensive review on the antecedents of AL must incorporate genetic underpinnings involved in diathesis-stress models. Genetics provide insights into the interaction between stress exposure and the regulation of internal biological processes across an individual's lifespan and across generations. A rich literature on the genetics involved in stress-related diseases is now reviewed to encourage future investigations.

Twin studies

Twin studies have demonstrated significant genetic contribution to basal cortisol secretion (Linkowski et al., 1993; Steptoe, van Jaarsveld, Semmler, Plomin, \& Wardle, 2009), stressreactivity (Steptoe et al., 2009), and other biomarkers commonly employed in the AL model (De Geus, Kupper, Boomsma, \& Snieder, 2007; Williams, Blanche, \& Krauss, 2005). In a study assessing salivary cortisol in unaffected twins discordant for affective disorders (Vinberg, Bennike, Kyvik, Andersen, \& Kessing, 2008), it was found that high-risk twins displayed elevated evening cortisol levels compared to low-risk twins. Notably, the high-risk twins in this study previously reported signs of discrete cognitive dysfunction (Christensen, Kyvik, \& Kessing, 2006) and a tendency for higher levels of neuroticism (Vinberg, Mortensen, Kyvik, \& Kessing, 2007). Overall, twin studies suggest that genetic factors control the expression of HPA-axis activity.

Although findings are mixed (Kirschbaum, Wust, Faig, \& Hellhammer, 1992), quantitative genetic modeling has shown significant heritability for cortisol reactivity to a stressor (Steptoe et al., 2009). In a recent meta-analysis that evaluated all published twin studies that assessed heart rate or blood pressure reactivity to stress exposure, the pooled heritability estimates ranged from $26 \%$ to $43 \%$ for reactivity to psychosocial stress (i.e., mental stressor) and $21 \%$ to $55 \%$ for reactivity to physiological stress (i.e., cold pressor test) (Wu, Snieder, \& de Geus, 2009). These 


\section{Transdisciplinary Allostatic Load Review}

studies highlight the importance of genetics as an underlying mechanism in individual differences in stress reactivity.

In uncovering the molecular pathway between genetic variability and AL, twin studies have examined the genetic underpinnings of additional biological variables found within the AL model. In a study of Swedish elderly twins, a dominant genetic component was reported for lowdensity lipoprotein, glucose, $\mathrm{Hb}_{\mathrm{AlC}}$, and C-reactive protein (Rahman et al., 2009). In a study assessing genetic and environmental contributions to cardiovascular disease and metabolic syndrome (Elder et al., 2009), it was reported that cholesterol and triglycerides were highly heritable (56\% to $77 \%$ ), and waist circumference, glucose, insulin, insulin resistance, and blood pressure were moderately heritable ( $43 \%$ to $57 \%$ ). Common environmental factors contributed to $23 \%$ to $40 \%$ of the variables of waist circumference, SBP, and glucose, suggesting a dominant genetic influence on total variance of factors associated with disease. While twin studies provide important information, the magnitude of heritability reveals little about the genetic structure of particular endophenotypes and plausible number of genes involved.

\section{Genetic association studies in humans}

Association studies have provided essential information on how specific genes contribute to the regulation of key biological processes involved in how sensitive individuals are likely to respond to stressors. Reports of gene-environment interactions demonstrate just how variation in genetic profiles interact with environmental stimuli, resulting in differential health outcomes or multifinality (Cichetti \& Toth, 2009). In accordance with the stress-diathesis model, individual differences at the genetic level may alter primary effects and secondary outcomes, resulting in varied susceptibilities to tertiary outcomes. Over the years, a number of genetic polymorphisms have been assessed in association with stress and stress-related disease in humans, including the 


\section{Transdisciplinary Allostatic Load Review}

serotonin transporter polymorphism (5-HTTLPR: short/long) (Caspi et al., 2003), brain derived neurotrophic factor (BDNF: Met66Val)(Vinberg et al., 2009), apolipoprotein E (APOE: epsilon e4) (Fiocco, Poirier, Joober, Nair, \& Lupien, 2008), and the catechol-O-methyltransferase gene (COMT: Met158Val)(Yavich, Forsberg, Karayiorgou, Gogos, \& Mannisto, 2007).

The 5-HTTLPR gene has been widely investigated for its role in affective disorders. Studies show that individuals with the short allele display greater stress-reactivity to psychosocial stress, with the greatest response in those homozygotic for the short allele (Gotlib, Joormann, Minor, \& Hallmayer, 2008; Way \& Taylor, 2010). Further, it is reported that the short allele interacts with stressful life events to increase risk of depression (Caspi et al., 2003; Kendler, Kuhn, Vittum, Prescott, \& Riley, 2005) and neuronal activation of brain regions involved in depression (Canli et al., 2006). The 5-HTTLPR has also been shown to moderate the association between lipid profiles and depression prevalence in older adults, with the association apparent only in homozygotic carriers of the short allele (Kim et al., 2009). On the other hand, a recent meta-analysis failed to conclude that the 5-HTTLPR genotype alone or in interaction with stressful life events is associated with an elevated risk of depression regardless of sex (Risch et al., 2009). Genetic polymorphisms may thus affect the magnitude of AL acquired over time as an individual faces and responds to environmental challenges, which then determines risk of adverse psychological and health sequelae associated with biological concomitants of the AL model.

COMT catalyzes the degradation of catecholamines like dopamine in the brain (Yavich et al., 2007) and is, as will be elaborated in a separate section, linked to psychotic disorders (Craddock, Owen, \& O'Donovan, 2006). The gene that codes for COMT contains a functional common Val158Met polymorphism, with the Val allele exhibiting a three- to four-fold increase in enzyme activity compared to the Met allele (Chen et al., 2004). Recent studies show that, compared to Val/Val carriers, healthy adults with the Met/Met genotype display a diminished 


\section{Transdisciplinary Allostatic Load Review}

opiod system response and greater sensory and affective ratings of pain following a sustained pain challenge (Zubieta et al., 2003) and increased limbic and prefrontal activation following the presentation of unpleasant stimuli (Smolka et al., 2005). These studies suggest that the Met allele lowers physiological and psychological resilience against negative environmental challenges, which may increase the risk of stress-induced disease.

In a study that assessed reactivity to a psychosocial stressor in healthy controls in risk

probands and major depressive disorder patients, an association between COMT genotype and stress reactivity was reported (Jabbi et al., 2007). This association differed depending on group status: Met/Met carriers displayed greater cortisol and adrenalin reactivity following the stressor. Yet, in at risk probands, the $\mathrm{Val} / \mathrm{Val}$ genotype group displayed greater reactivity. No association was found in the patient group; however, this was attributed to current medication use. This study presents the possibility that epigenetic mechanisms are at play: risk exposure alters the function of the gene, which then biases an individual's response to subsequent stressors.

\section{Epigenetics}

Epigenetics examines changes in DNA methylation and histone modifications produced by perturbations in the environment (e.g., early adversity) that in turn alter the function, but not the structure, of a gene. Research shows that changes incurred through epigenetics are stable and can even be transmitted inter-generationally (Meaney \& Szyf, 2005). While a majority of epigenetic findings stem from animal research (Francis, Diorio, Liu, \& Meaney, 1999; Szyf, Weaver, Champagne, Diorio, \& Meaney, 2005; Weaver et al., 2004), two studies have thus far evaluated the epigenetic model in humans.

A twin study that examined the genetic and environmental contributions to cortisol reactivity in a cohort of 19-month-old twins from high and low family adversity found that high 


\section{Transdisciplinary Allostatic Load Review}

family adversity has a differentiation developmental effect on cortisol reactivity (Ouellet-Morin et al., 2008). Specifically, both genetic and environmental factors explained the variance in cortisol reactivity to a mild stressor (i.e., unfamiliar situation) for twins from low family adversity. By contrast, variance in cortisol reactivity was only explained by shared and unique environmental factors in twins who had experienced high family adversity (characterized as having four of the following; maternal smoking during pregnancy, low birth weight, low family income, low maternal education level, single parenthood, young motherhood, and maternal hostile or reactive behaviors). As discussed earlier, infants exposed to adverse environments likely undergo alterations in their biological embedding over and above any genetic contributions, and yet the pathways driving stress responsivity can differ markedly from one family context to the other. Until more research is conducted, the precise manner whereby such mechanisms promote resilience or vulnerability is speculative, but might involve differential neurodevelopmental processes.

Epigenetic differentiation precipitated by early adversity might effect GC gene promotor expression in the hippocampus via DNA methylation. A recent postmortem study (McGowan et al., 2009) assessing the epigenetic regulation of the GC receptor in suicide victims with or without a history of childhood abuse reported differential neuron-specific GC receptor promotor in postmortem hippocampi. Specifically, decreased levels of GC receptor mRNA and increased cytosine methylation of the GC receptor promotor was found in abused suicide victims. Early adversity can also induce modifications in the methylation status of the BDNF gene, which can be transmitted from one generation to the next (Roth, Lubin, Sodhi, \& Kleinman, 2009). While these studies are tips of the iceberg, they nonetheless suggest that genetic effects and epigenetic interactions might influence AL susceptibilities via physiological recalibrations of the HPA-axis and regulatory neural substrates. 


\section{Transdisciplinary Allostatic Load Review}

\section{Genetic study of allostatic load}

To date, only one study has directly assessed the association between genetic variance and AL (Smith et al., 2009). In a population-based study of chronic fatigue syndrome, 182 Caucasian participants were genotyped and an AL index was calculated, based on measures representing metabolic, cardiovascular, inflammatory, HPA-axis and autonomic activities. Polymorphisms in 32 candidate genes related to HPA-axis activity, neurotransmitter systems, inflammation, cardiovascular, and metabolic functions were analyzed. After correcting for covariates and multiple comparisons, genetic polymorphism of the gene that codes for angiotensin-I converting enzyme (ACE C/T: rs4968591) remained significantly associated with AL status. Specifically, participants with the T allele of the ACE gene were more likely to display a higher AL index than participants with the $\mathrm{C}$ allele.

ACE catalyses the conversion of angiotensin-I (Ang-I) to Ang-II, which is both proinflammatory and pro-oxidant and thus plays an important role in cardiovascular, endocrine, and immune functions (Garg \& Yusuf, 1995; Malik et al., 1998; Maschio et al., 1996). The ACE enzyme further converts Aß42 to Aß40 in the brain (Zou et al., 2009), which is implicated in the

development of neuronal plaques of particular concern in older life dementias. Indeed, polymorphism of the ACE gene has been associated with type-II diabetes mellitus (Stephens et al., 2005), obstructive pulmonary disease (Tkacova et al., 2005), and Alzheimer's disease (Kolsch et al., 2005). As the common ACE polymorphism in these studies (ACE I/D) is not in linkage disequilibrium with the ACE T/C gene, additional prospective research is needed to confirm the association between $\mathrm{ACE}$ and $\mathrm{AL}$ and any functional, long-term consequences.

\section{Conclusion \& future directions}




\section{Transdisciplinary Allostatic Load Review}

Future studies should go beyond the basic stress-diathesis structure and consider genetic and epigenetic influences on biological systems that are integral to the AL model. Future investigations of genetic mechanisms contributing to AL such as telomere length as tertiary outcomes would be immensely informative (Epel, 2009; Epel et al., 2004; Sapolsky, 2004). In addition, studies incorporating multiple dimensions of outcomes (biological, psychological, social, behavioral, spiritual) and global self-perceptions of health (Jylha, 2009) would help us determine how genetic and epigenetic factors interact with other systems and with individual's health in general to promote vulnerability or resilience. Indeed, a more comprehensive understanding of the (epi)genetic regulatory mechanisms and their relationship with chronic stress should help inform individualized prevention strategies for reducing or minimizing the underpinnings of AL.

Like the case of the orchid child discussed previously, it is imperative to understand how protective factors can be harnessed to dampen the effects of risk factors like harmful genetic makeups and/or early adversities. For instance, an exemplary review of gene-stress-behavior interactions in adolescent alcohol use outlines how to intervene early to minimize the likelihood that inherited vulnerabilities towards alcoholism and associated psychopathologies before morbidities manifest themselves (Zimmermann, Blomeyer, Laucht, \& Mann, 2007). The interface between individuals and foreign substances that endanger health (e.g., cigarettes) is an important avenue to pursue with respect to stress research. And yet, these have had little place in the AL literature other than as behavioral covariates in statistical equations. As numerous noxious factors knowingly and unknowingly enter our bodies to exert deleterious effects, we would do well to determine the precise mechanisms of their actions on the brain and body. To address this lacuna, we now turn to how environmental toxins can directly or indirectly affect AL throughout life. 


\section{Environmental Toxins: Mediators or Moderators of Allostatic Load?}

Genetic variance exists in an individual's ability to absorb, distribute, metabolize, and excrete environmental toxins. For example, genotypes associated with heavy metal absorption and toxicity include hemochromatosis (Wright et al., 2004), vitamin D receptor (Chuang et al., 2004), and apolipoprotein-E (Godfrey, Wojcik, \& Krone, 2003). Environmental toxins (ETs) are

principally industrial chemicals like lead $(\mathrm{Pb})$, methylmercury $(\mathrm{MeHg})$, and polychlorinated biphenyls (PCB) that can contribute to functional disruptions, neurodevelopmental delays, behavioral as well as cognitive impairments throughout the lifespan (Bradley \& Corwyn, 2002; Furumoto-Dawson, Gehlert, Sohmer, Olopade, \& Sacks, 2007). Unfortunately, ETs have received little attention in the AL model, and yet they have the propensity to potently effect AL biomarkers by means of mediation or moderation effects at differential periods throughout lifespan development (see Table 2).

\section{Vulnerable populations to environmental toxins exposure}

While everyone is vulnerable to ETs, children and older adults are particularly sensitive to exposure. Extensive studies of $\mathrm{Pb}, \mathrm{MeHg}$, and $\mathrm{PCBs}$ reveal multiple mechanisms by which these compounds disrupt normal brain development (Landrigan, 1999; Stein, Schettler, Wallinga, \& Valenti, 2002). Children also have greater exposure to ETs than adults in terms proportioned body weight by way of water consumption, food, and air, as well as through increased hand-to-mouth and playground behaviors.

Aging individuals are also highly vulnerable to a lifetime's worth of accumulated ETs that can exacerbate senescence. For instance, toxicokinetic studies have demonstrated that $95 \%$ of $\mathrm{Pb}$ is stored in the skeleton only to then be released into circulation during age-related bone demineralization (Rabinowitz, 1991). Data from the National Health and Nutrition Examination 


\section{Transdisciplinary Allostatic Load Review}

Survey (NHANES) confirm that adults over the age of 50 have the highest blood Pb levels (Pirkle et al., 1998). Moreover, our elders have much higher exposure to ETs than younger generations benefitting from recent bans and/or restrictions. In sum, older adults and youths are extremely vulnerable to ETs that can, like chronic stress, increase sensitivities towards disrupted development and accelerated brain declines (Lupien et al., 2009).

\section{Environmental toxins as endocrine disruptors}

One plausible pathway whereby ETs impact the body and the brain is through interactions with the endocrine system. Early observations of reproductive organ malformations and aberrant sexual behaviours in wildlife first unveiled the ability of ETs to interfere with steroid hormones. This led to their infamous classification as endocrine disrupting chemicals (Hotchkiss et al., 2008; Zala \& Penn, 2004), which the World Health Organization defines as "an exogenous substance or mixture that alters function(s) of the endocrine system and consequently causes adverse health effects in an intact organism, or its progeny, or (sub)populations" (WHO, 2002). Identified environmental estrogens and anti-estrogens include certain heavy metals ( $\mathrm{Pb}$, cadmium, $\mathrm{MeHg}$ ), industrial chemicals (PCBs, dioxins and furans), pesticides (DDT, Organophosphates), herbicides (Atrazine), and plasticizers (Bisphenol A, surfactant) (Darbre, 2006; Weiss, 2007) that are dangerous even at very low doses (Denham et al., 2005; Jacobson \& Jacobson, 1996).

As ETs are ubiquitous, affect particularly children and elders, and interact with endocrine functioning, they should be addressed by the AL model. In the following paragraph, we will examine whether environmental toxins might (1) directly impact the HPA-axis; (2) potentiate other chronic stressors; and/or (3) effect individual AL biomarkers.

\section{Environmental toxins and allostatic load}




\section{Transdisciplinary Allostatic Load Review}

While scarcely studied in humans, ETs are believed to function as GC receptor antagonists (Johansson, Nilsson, \& Lund, 1998) and can alter GC biosynthesis in adrenocortical cells (Li \& Wang, 2005). Animal studies reveal that dysregulated HPA-axis responses to acute stressors are related to heavy metal exposure (cadmium, lead, mercury) and organic environmental contaminants (PCBs, solvents, pesticides) in fish (Brodeur, Sherwood, Rasmussen, \& Hontela, 1997; Hontela, Dumont, Duclos, \& Fortin, 1995), amphibians (Gendron, Bishop, Fortin, \& Hontela, 1997), birds (Love et al., 2003), rodents (Meserve, Murray, \& Landis, 1992; Vyskocil, Fiala, Ettlerova, \& Tenjnorova, 1990), and large mammals (Oskam et al., 2004; Zimmer et al., 2009). In humans, even low levels of $\mathrm{Pb}$ exposure correspond to increased cardiovascular (Gump et al., 2005) and cortisol reactivity to the cold pressor task in 9-10 year-old children (Gump et al., 2008). As the cold pressor task is primarily a physical stressor, it will be important to replicate these findings using psychosocial stressors that elicit social-evaluative threats known to induce greater HPA-axis activations than other laboratory tasks (Dickerson \& Kemeny, 2004).

ETs may directly impair the HPA-axis or alternatively act as mediators of chronic stressors like poverty. Indeed, ET exposures are demographically constrained, occurring most frequently in socio-economically disadvantaged environments (Sexton, 1997). For instance, children living in older houses containing Pb paint (Pirkle et al., 1998) and/or living in the center of large cities are exposed to greater Pb levels than those living more peripherally (Brody et al., 1994; Pirkle et al., 1994; Sexton et al., 2006). Impoverished individuals are also more likely than affluent ones to live near environmental hazards like industrial plants, reside in urban areas where ambient levels of many air pollutants tend to be higher, eat significantly greater amounts of contaminated fish, and be employed in potentially dangerous occupations (Sexton et al., 2000).

In addition, high $\mathrm{Pb}$ levels could be a mechanism whereby family income effects HPA-axis functioning in children (Gump et al., 2009). Using data from the Oswego study, increased early 


\section{Transdisciplinary Allostatic Load Review}

childhood blood $\mathrm{Pb}$ levels significantly mediated the association between decreased family income and augmented salivary cortisol levels following acute stress. While this might denote a meditational effect, the direction of the association is indiscernible, as reverse causality remains a possibility: adrenocortical reactivity may have effects on the toxicokinetics of blood to bone transfers of $\mathrm{Pb}$ (Mushak, 2003).

ETs might also indirectly synergize HPA-axis dysregulations and increase vulnerabilities towards inter-generational transmission of psychopathologies. A notable American population study recently found that background levels of $\mathrm{Pb}$ were associated with increased odds of developing major depression in 20-40 year old adults (Bouchard et al., 2009). Interestingly, this age range corresponds to the average reproductive years of humans. Our group has previously shown that children with increased cortisol levels are more likely to have depressed mothers and come from disadvantaged neighborhoods (Lupien, King, Meaney, \& McEwen, 2000). It follows that living in lower socio-economic strata with increased $\mathrm{Pb}$ exposure could directly impact children's HPA functioning or indirectly via the mother's mental health, or perhaps a mix of both. At a transdisciplinary level, such complex interactions among environmental, biological, and psychosocial factors in developing children could result in an altered state of global health that could lead to psychopathologies and concomitant co-morbidities. The interactions of ETs with other types of factors (e.g., early adversity, genetic endowment) may thus lead to complex dysregulations at multiple levels that consequently render the individual more susceptible to the effects of chronic stress by enhancing the toll of AL.

In mechanistically considering ETs in interaction with AL, only one group have systematically assessed the combined effects of ETs and stressors using the rodent model (CorySlechta et al., 2008). Based on the premise that $\mathrm{Pb}$ exposure acts on the mesocorticolimbic dopamine pathway (Zuch, O'Mara, \& Cory-Slechta, 1998) in semblance to products of the HPA- 


\section{Transdisciplinary Allostatic Load Review}

axis, Cory-Schleta and colleagues (2008) confirmed that Pb and GCs interact in this tract. By assigning dams to four experimental groups as a function of $\mathrm{Pb}$ exposure (waterborne 2 months before breeding and into lactation vs. not) and maternal stress (restraint at gestational day 16 and 17 vs. not), they observed behaviors dependent on this pathway (e.g., locomotion, impulsivity) in 60-day old offspring. Results showed that the dams exposed to combined $\mathrm{Pb}$ and restraint had female offspring with higher corticosterone levels than contrast groups (Cory-Slechta, Virgolini, Thiruchelvam, Weston, \& Bauter, 2004). In a subsequent study that limited the timing of $\mathrm{Pb}$ exposure to post-weaning periods, it was shown that interactions among $\mathrm{Pb}$ and stressors are not restricted to maternally mediated exposure (Virgolini, Chen, Weston, Bauter, \& Cory-Slechta, 2005). Even at low levels (10 ug/dL), Pb exposure interacts with prenatal stress to further modify both behavioral and corticosterone responses to stressors (Virgolini, Rossi-George, Weston, \& Cory-Slechta, 2008). These findings translate into a major danger for population health. In the United States, the acceptable childhood blood $\mathrm{Pb}$ threshold is currently $10 \mathrm{ug} / \mathrm{dL}$. Yet amidst public health efforts to restrict exposure, $1.6 \%$ of American children ages 1 to 5 have levels greater than or equal to $10 \mathrm{ug} / \mathrm{dL}$ (Centers for Disease \& Prevention, 2005; Jones et al., 2009).

As far as we know, only one epidemiological study has reported interactive effects of stress and $\mathrm{Pb}$ in the general population (Peters et al., 2007). In the Normative Aging Study, individuals with increased psychological stress, in addition to cumulative $\mathrm{Pb}$ exposure, had an increased risk of developing hypertension independent of standard covariates. Specifically, those reporting high stress levels had 2.65 times greater chance of developing hypertension per standard deviation increase in $\mathrm{Pb}$. These results strongly suggest that the effect of $\mathrm{Pb}$ on hypertension interacts with chronic stress levels independent of demographic and behavioral risk factors. Apart from $\mathrm{Pb}$, ETs may exert their influence on the HPA-axis through various pathways: (1) directly by affecting the HPA-axis (2) indirectly by mediating the effects of environmental factors on the 


\section{Transdisciplinary Allostatic Load Review}

HPA-axis functioning, and (3) indirectly by potentiating the effects of maternal stress on offspring HPA-axis functioning and behavioral outcomes. These pathways may represent an additional form of AL that can increase the probability of developing physical and mental disease. While ETs have been proposed as mediators or moderators of AL (Loucks, Juster, \& Pruessner, 2008), no corresponding studies exist and so we must therefore turn to studies of individual AL biomarkers.

\section{Environmental toxins as disrupters of individual allostatic load biomarkers}

In addition to neuroendocrine effects, ETs can impair cardiovascular, immune, and metabolic functions. A positive correlation between $\mathrm{Pb}$ exposure and blood pressure has been identified in numerous studies (Fewtrell, Pruss-Ustun, Landrigan, \& yuso-Mateos, 2004; Gump et al., 2005; Nash et al., 2003; Navas-Acien, Guallar, Silbergeld, \& Rothenberg, 2007). Increased vulnerabilities for cardiovascular morbidities and mortality have even been shown at lead levels < $5 \mu \mathrm{g} / \mathrm{dL}$ in large epidemiological studies (Menke, Muntner, Batuman, Silbergeld, \& Guallar, 2006; Muntner, Menke, DeSalvo, Rabito, \& Batuman, 2005). Concerning MeHg toxicity, most studies have been conducted in populations dependant on fish as a primary food source, such as in the Seychelles study (Davidson et al., 1998; Myers et al., 1997) or the Faroe Island study (Debes, Budtz-Jorgensen, Weihe, White, \& Grandjean, 2006; Grandjean et al., 1992).

While controversial (Chan \& Egeland, 2004; Stern, 2005), several reports substantiate an adverse association between $\mathrm{MeHg}$ exposure and relative risk of myocardial infarction (Salonen et al., 1995) and hypertension (Fillion et al., 2006). Likewise, PCB 126 (a dioxin-like compound) exposure in female rats increases cardiovascular risk factors like hypercholesterolemia and hypertension (Lind, Orberg, Edlund, Sjoblom, \& Lind, 2004). One human study even showed that Native Americans with increased PCB levels had an increased risk of developing cardiovascular disease through increased synthesis of cholesterol and triglycerides (Goncharov et al., 2008). 


\section{Transdisciplinary Allostatic Load Review}

Although the immunotoxic mechanism of ETs is not well understood, deleterious effects have been observed in animals (Esser \& Welzel, 1993) and humans (Baccarelli et al., 2002; Heilmann, Grandjean, Weihe, Nielsen, \& Budtz-Jorgensen, 2006). An accumulating body of evidence suggests that in-utero ET exposure might increase susceptibility to infections in young children (Dallaire et al., 2006; Weisglas-Kuperus, Vreugdenhil, \& Mulder, 2004). In the NHANES, exposure to monohydroxy polycyclic aromatic hydrocarbons (derived from incomplete combustion of organic matter and fossil fuels including diesel engines, domestic heating, and pyrolysis of coal or tobacco smoke) was associated with increased C-reactive protein (Everett et al., 2010). In the previous phase of the NHANES study, exposure to cadmium, which is used as a pigment and plastic stabilizer, was also positively associated with increased plasma C-reactive protein and fibrinogen levels (Lin, Rathod, Ho, \& Caffrey, 2009), while Pb exposure was associated to inflammatory biomarkers (Songdej, Winters, McCabe, \& van Wijngaarden, 2010).

As mentioned before, ETs also increase lipid profiles (Goncharov et al., 2008). Specifically, PCBs and other dioxin-like components are associated with increased risk factors representative of metabolic syndrome in Americans (Lee, Lee, Porta, Steffes, \& Jacobs, 2007b) and Japanese (Uemura et al., 2009) alike. Notwithstanding, differential ET exposures are based largely on socio-economic as well as cultural differences both within and between populations that should be considered by future investigations.

\section{Conclusion and future directions}

Because several AL biomarkers are effected by or interact with ETs, we believe that they should be explored as mediators and/or moderators in the AL model. In particular, elucidating how ETs effect developmental trajectories towards pathological outcomes from childhood until old age will be important to consider. We have limited this review to the best-characterized ETs 


\section{Transdisciplinary Allostatic Load Review}

$(\mathrm{Pb}, \mathrm{MeHg}$, and $\mathrm{PCBs})$, but many other chemicals currently used in numerous commercial goods are identified or suspected endocrine disrupters with neurotoxic effects. Furthermore, the majority of ETs reviewed are endocrine disrupting chemicals that alter HPA axis and sex hormone functions in humans (Agusa et al., 2007).

ETs are likely also effected by numerous other factors (psychosocial, biological, behavioral). Studying the impact of ETs on psychopathology could be improved by adopting a transdisciplinary approach that focuses on the interaction of ETs with multiple levels of outcomes

existing in parallel. For instance, are the sexes exposed differently to ETs based on gender-typed work environments (e.g., factories, primary industries), and how can ETs interact with sex hormones to disrupt physiology? We are far from being able to accurately respond to such questions. Just in terms of sex differences in ET exposures, genetic interactions, and early adversities, much remains unknown, as the concept of "sex" is complex but powerful in determining patterns of health and disease. In order to facilitate answers for future inquiries in ETs research and all the aforementioned litarature, we next explore nuances among one's biological sex, one's socio-culturally generated gender identity and behaviors, and the modulating effects of sex hormones on AL levels.

\section{Sex, Gender, and Sex Hormones}

One's sex confers a unique set of vulnerabilities to specific disease pathways. Women are more prone to affective and autoimmune disorders, whereas men are more at risk of early mortality, substance abuse, conduct/antisocial disorder, and infectious diseases. While men are more stress responsive to acute stressors (Kajantie \& Phillips, 2006; Kirschbaum, Wüst, Hellhammer, 1992; Kudielka \& Kirschbaum, 2005), women experience greater subjective distress, perhaps due to socio-cultural factors. As this section will elucidate, biological sex, gender-roles, 


\section{Transdisciplinary Allostatic Load Review}

and sex hormones are elusively linked to AL levels.

The term sex refers to the biological differences between men and women such as genetic sex (female XX and male XY chromosomes) and hormonal milieu. For instance, men have higher testosterone levels and women have higher estrogen and progesterone levels. These sex steroids have both organizational and activational effects that shape sex differences ranging from morphology to behavior. Organizational effects refer to permanent actions during a critical early developmental period that modulate brain responses to certain stimuli later in life. On the other hand, activational effects refer to transient physiological and/or behavioural responses triggered by the activation of the matured gonadal axis occuring at any age thereafter.

Lifelong dynamics and exposure to sex steroids differ greatly between sexes: men experience an age-related linear decline in testosterone levels whereas women's lives are characterized by constant periodic fluctuations including menarche, the menstrual cycle, pregnancies, and menopause. Consequently, when it comes to AL, the biological sex differences observed might depend on sex steroids (i.e., testosterone vs. estrogens), levels of sex steroids (i.e., phase of the menstrual cycle, pregnancy, menopause), and previous or lifelong exposures (i.e., age at menarche/puberty, number of pregnancies, age at menopause, exogenous administration such as hormonal contraceptive and replacement therapy). As differences in the hormonal milieu vary greatly between individuals throughout a lifetime, a simple dichotomized perspective of sex differences might be oversimplified. Incorporating validated measures of the most important dynamic variables not homogenously distributed across sexes could indeed refine the current dichotomous classification of sex.

Men and women also differ in terms of their socialization, identity, and subjective roles that are expected of them within given socio-cultural periods (Lips, 2008). Gender or sex-roles refer to the implicit and explicit dissimilarities in an array of socio-culturally constructed roles and 


\section{Transdisciplinary Allostatic Load Review}

relationships as well as sex-typical personality factors (e.g., assertiveness). To date, little is known of the impact of gender on stress, although various gender-specific and health-related behaviors confer a differential vulnerability or resilience to various diseases within and between societies (Courtenay, 2000). In terms of stress responsivity, a study found that men tend to benefit from their partner's social support by secreting decreased cortisol levels when facing an acute stressor whereas women had an increased cortisol response in the presence of their partner but not in the presence of a stranger (Kirschbaum, Klauer, Filipp, \& Hellhammer, 1995).

Gender might also be linked to diverse patterns of threat processing and coping mechanisms. Indeed, women demonstrate increased cortisol levels when facing social rejection challenges whereas men tend to be more reactive when confronted with achievement-based stressors (Stroud, Salovey, \& Epel, 2002). As tasks that elicit socio-evaluative threat induce HPAaxis activations (Dickerson \& Kemeny, 2004), differences in stress perception might be driven by social constructs and gender-based attributions. Substantiating this is a fascinating evolutionary argument hypothesizing that men and women cope differently to stress. Accordingly, the primary stress response pattern for men is the "fight-or-flight" response, whereas women might be more prone to engage "tend-and-befriend" mechanisms, such as nurturing and socializing behaviors that may protect against the demands of pregnancy, nursing, and child care (Taylor et al., 2000). This idea might shed some light on sex differences found in the AL literature that are moderated by social and relational factors. Taken from a developmental psychopathology perspective, interactions among sex and gender force us to reconsider how dichotomous and dimensional variables co-exist to affect a wide spectrum of behaviors, cognitions, and life-long propensities towards certain diseases.

Allostatic load sex differences 


\section{Transdisciplinary Allostatic Load Review}

The MacArthur studies provide evidence for age-specific sex differences in AL (Juster et al., 2010). High cortisol levels are associated with memory impairment in women (Seeman et al., 1997a), while elevated noradrenalin levels relate to cognitive decline in men (Karlamangla, Singer, Chodosh, McEwen, \& Seeman, 2005a). Using advanced statistical techniques to tease apart biomarker combinations demarcating 12-year mortality risk between the sexes, Gruenewald and colleagues (2006) found that high risk pathways of biomarker clustering for men included adrenalin, noradrenalin, interleukin-6, C-reaction protein, and fibrinogen, while for women included interleukin-6, C-reactive protein, $\mathrm{Hb}_{\mathrm{Alc}}$, and systolic blood presure (Gruenewald et al., 2006). Interestingly, elevated systolic blood pressure occurred in $100 \%$ of female high-risk pathways but only $17 \%$ for male high-risk pathways principally dominated by elevated fibrinogen, noradrenline, and adrenalin levels otherwise completely absent for females.

Such "biological signatures" demonstrate that there are multiple sex specific routes whereby exacerbated biomarkers lead to AL and mortality risk. In an earlier analysis, however, cardiovascular biomarkers were more often dysregulated for males, while neuroendocrine biomarkers were more often dysregulated for females (Seeman, Singer, Ryff, Dienberg Love, \& Levy-Storms, 2002). These findings from the same cohort measured at different time-points reveal that AL pathways indeed differ between the sexes, although sex-specific biomarker clusters depend on age and perhaps sex hormones levels. Note that AL sex differences may not be as easily detected in younger cohorts. For instance, a recent 15-year follow-up study using the Coronary Artery Risk Development in Young Adults (CARDIA) study did not find evidence for sex nor ethnicity differences in forty year old participants on average (Seeman et al., 2009). Noteworthy is that the authors' application of structural equal modeling of AL biomarkers accounted for as much $84 \%$ of explained variance among heart rate variability, blood pressure, inflammatory, metabolic, catecholaminergic, and GC meta-factors. It would be interesting to see 


\section{Transdisciplinary Allostatic Load Review}

how clusters of AL biomarkers differ between the sexes in follow-ups of other cohort studies, and to what extent the social and cultural environment moderates potential effects throughout the life cycle.

Early life experiences, SES, and ethnicity further moderate AL among the sexes. Retrospective reports of positive parental bonding, harmonious later life relationships and the presence of a spouse are associated with lower AL in elderly men who benefit most from emotional support (Seeman et al., 2004; Seeman et al., 2002). On the other hand, low SES results in elevated levels of AL in African American women (Geronimus, Hicken, Keene, \& Bound, 2006) and female sex leads to higher AL with higher type II diabetes risk in American Samoan women (Crews, 2007). Social ties with close friends is protective in both elderly women and men (Seeman et al., 2004), while concurrent and cumulative perceived stress increases AL in both sexes, but it is most strongly expressed in women (Goldman et al., 2005). Interestingly, decreased AL levels have been reported in women but not men who regularly attend religious services (Maselko, Kubzansky, Kawachi, Seeman, \& Berkman, 2007) and who have a greater sense of coherence and meaningfulness in life (Lindfors, Lundberg, \& Lundberg, 2006). These studies highlight the importance of sex in the assessment of AL biomarkers and perhaps as well to the existential and spiritual benefits conferred on particular gender-typed behaviors.

Taken together, such findings corroborate important interactions known to exist between spiritual factors such as sense of purpose, meaningfulness, connectedness, and other health domains (Picard et al., 2011) within the context of social and cultural elements. While these findings corrorabrate existing literature (Seeman, Dubin, \& Seeman, 2003), much concerning such multi-faceted interactions in relation to psychopathology remain to be elucidated. Most importantly, we must highlight that no AL studies have measured sex hormones, although their 


\section{Transdisciplinary Allostatic Load Review}

potential implications in various AL stages and disease trajectories is critical, as are their role in these sex differences.

\section{Male sex hormones}

Starting at the 3rd to 4th decade of life, testosterone (T) levels in men generally decrease at a proportion of 1 to $2 \%$ per year (Feldman et al., 2002). It is now well known that androgen levels have a series of impacts on different biological systems. Beyond reproductive functions, male sex hormones interact and even regulate some of the AL primary mediators and secondary outcomes that could potentially lead to some serious issues, especially in hypogonadal men.

The HPA-axis has an inhibitory effect on the hypothalamic-pituitary-gonadal (HPG)-axis at the detriment of reproductive behavior and other HPG tract functions (Viau, 2002). In humans, increased GCs levels impair the expression of the luteinizing hormone-releasing hormone (LHRH), the secretion of luteinizing hormones (LH) and the synthesis of sex steroids like $\mathrm{T}$ (Rivier \& Rivest, 1991; Tilbrook, Turner, \& Clarke, 2000). However, the relationship between these two endocrine systems is not unidirectional. While cortisol has inhibitory effects at all three levels of the HPG-axis, T itself inhibits HPA-axis activity at the hypothalamic level (Terburg, Morgan, \& van Honk, 2009), exerting a strong influence on GC regulation that may modulate sex differences in stress-related HPA activity (Kerr \& Kerr, 2001; Kornstein et al., 2000). In a variety of species, it is well known that males show lower ACTH and GC levels in both basal and stressinduced HPA-axis activity due, in part, to the inhibitory effects of T (Handa, Burgess, Kerr, \& O'Keefe, 1994a; Young, 1998). Moreover, in a male rat model of stress, gonadectomy increases $\mathrm{ACTH}$ and corticosterone responses to acute stress while 5-alpha-dihydrotestosterone (5 $\alpha$-DHT) replacement reverses this effect; this modulatory effect has not, however, been addressed under conditions of chronic stress (Handa et al., 1994b). 


\section{Transdisciplinary Allostatic Load Review}

There is also a strong relation between $\mathrm{T}$ and cytokine production. Normal aging is associated with increasing levels of cytokines including interleukin-6 (IL-6) and tumor necrosis factor-alpha (TNF- $\alpha)$ during the same time period as age-related $\mathrm{T}$ decreases. There is evidence that both IL-6 and TNF- $\alpha$ have an influence on the three levels of the HPG-axis, resulting in impaired synthesis and release of T (Maggio et al., 2005; Mealy, Robinson, Millette, Majzoub, \& Wilmore, 1990). Indeed, higher plasma cytokine levels have been observed in men with lower androgen levels (Pugh, English, Jones, \& Channer, 2000). On the other hand, testosterone treatment has been reported to reduce the quantity of circulating pro-inflammatory cytokines. In an in vitro and in vivo experimental study, Pugh and coworkers (2000) found that androgens suppress macrophage production of cytokines and that gonadotropin therapy reduces the high levels observed in hypogonadal men. These findings suggest that exogenous $\mathrm{T}$ may be used as a potential anti-inflammatory, although further research must be done to confirm the causality between inflammatory markers and $\mathrm{T}$ in aging men.

In terms of secondary outcomes, $\mathrm{T}$ deficiency has been shown to alter vascular smooth muscle reactivity, which results in vasoconstriction (Traish, Guay, Feeley, \& Saad, 2009a) and is further shown to result in significant reductions in blood pressure in 66 hypogonadal men with intramuscular testosterone therapy (Zitzmann \& Nieschlag, 2007). Several studies have also reported that low $\mathrm{T}$ levels are associated with increased total cholesterol and LDL-cholesterol, while androgen treatment results in a favorable lipid profile. $\mathrm{T}$ additionally has a positive association with HDL-cholesterol levels, although there are some discrepancies when it comes to remediation by means of $\mathrm{T}$ treatment (Traish, Saad, \& Guay, 2009c). Low $\mathrm{T}$ levels also correspond to elevated insulin, glucose, and $\mathrm{Hb}_{\mathrm{Alc}}$ levels, which are reversed by androgen therapy (Traish, Saad, Feeley, \& Guay, 2009b). Several studies demonstrate that T supplementation in hypogonadal middle-aged men leads to a significant reduction of visceral fat, that acts like an 


\section{Transdisciplinary Allostatic Load Review}

endocrine organ promoting cytokine production, and a decrease of plasma insulin levels and increased glucose concentrations (Marin et al., 1992a; Marin, Krotkiewski, \& Bjorntorp, 1992b; Rebuffe-Scrive, Marin, \& Bjorntorp, 1991). A host of studies have come to conclude that clinically reduced $\mathrm{T}$ plasma levels lead to a higher risk of developing type-II diabetes, insulin resistance, obesity, atherosclerosis, metabolic syndrome, and cardiovascular disease (Traish et al., 2009b). In sum, androgen deficiency is associated with many pathophysiological states that modulate increased AL.

At a neurological level, $\mathrm{T}$ also acts as an endogenous neuroprotective factor in men independently from estrogen. Androgens promote neuron viability during neural development. In adult brains, $T$ treatment enhances nerve regeneration and attenuates neuron loss following injury or disease-related toxicity, especially in motor neurons (Jones, Pugh, Hall, Channer, \& Jones, 2003). In addition, androgens play a protective role in neuron survival in brain areas including the hippocampus and cortical regions particularly vulnerable to neurodegenerative diseases such as Alzheimer's disease (Simerly, Chang, Muramatsu, \& Swanson, 1990), functioning as an antiaging agent with beneficial regulation of pathogenic $\beta$-amyloid accumulations.

While there are still some inconsistencies in the literature, endogenous $\mathrm{T}$ levels effect cognition with reports of impaired memory and visuo-spatial functions in middle-aged and elderly men with lower T levels and conversely enhanced attention, executive function, processing speed, and global cognition in individuals with higher T levels (Warren, Serby, \& Roane, 2008). In a cross-sectional study with 400 men between the ages of 40 and 80 , a curvilinear association between $\mathrm{T}$ and memory performance and processing capacity was observed in the oldest group pointing to an optimal sex hormone level for cognitive functioning, although no explanatory mechanism has been proposed (Muller, Aleman, Grobbee, de Haan, \& van der Schouw, 2005). 


\section{Transdisciplinary Allostatic Load Review}

A relationship has also been observed between androgen deficiency and symptoms of major depression such as weakness, fatigue, insomnia, irritability, lack of concentration, and memory impairment. Although it is not clear that androgens have a direct effect on the development or maintenance of major depressive disorder, prevalence rates are higher in hypogonadal men (Amore, 2005). Furthermore, low testosterone values are associated with male depressive illness while androgen therapy seems to have antidepressant proprieties. It has been proposed that the heterogeneity in $\mathrm{T}$ acts on the HPA-axis and may explain the variability in individual resistance to antidepressant treatment (Holsboer \& Barden, 1996). These results identify a potential mechanism for the variability in psychopharmacological responsivity that should be explored further.

$\mathrm{T}$ levels may also play a crucial role in other psychiatric conditions, such as antisocial personality disorder (APD) and psychopathy. A large body of evidence has established the testosterone-cortisol ratio as a potential marker for aggressive tendencies. Indeed, high $\mathrm{T}$ levels combined with low cortisol values lead to more attention towards anger and confrontation, less inhibition of aggressive behavior, and less experienced fear (Terburg et al., 2009). These factors overlap with APD and psychopathy symptomatology and the ratio imbalance may be one of the mechanisms explaining social aggression in this personality disorder. Pharmaceutically readjusting and maintaining balanced $\mathrm{T}$ and cortisol levels might reduce antisocial and aggressive behaviors.

In summary, there is considerable evidence that $\mathrm{T}$ can be beneficial, especially in middleaged and elderly men as well as individuals suffering from hypogonadism. T replacement therapy may offer hope in improving numerous clinical conditions, as T influences AL at every level of dysregulation starting with primary mediators like GCs and cytokines, right through to tertiary outcomes including, among others, cardiovascular disease, type-II diabetes, atherosclerosis, and 


\section{Transdisciplinary Allostatic Load Review}

Alzheimer's disease. On the other hand, we must be very cautious in arriving at any hasty conclusions before further research establishes the specific role androgens exert on many interconnected biomarkers, modulators, and outcomes assessed using the AL model. At a transdisciplinary level, documenting time course patterns of interactions between $\mathrm{T}$ with respect to other life domains may yield important insights into the global health implications of abnormal $\mathrm{T}$ levels.

Female sex hormones

Like T, estrogens (E) have essential biological effects on many physiological processes. Although E are traditionally connected with female reproduction, the importance of these hormones in non-reproductive processes such as cardiovascular health, immune functioning, bone synthesis, and brain morphology has also been implicated with a plethora of mechanisms towards disease pathways.

There are several interactions between $\mathrm{E}$ and stress hormones that potentiate neuroprotection from cumulative stress perception and consequent HPA-axis dysregulation. The mechanisms underlying the cross talk between the gonadal and adrenal axis remain elusive, but evidence suggests that E might counteract the potentially damaging actions exerted on neural integrity and functioning (McEwen, 2002a).

Acute stimulation of the HPA-axis by either a physical or psychosocial stressors suggests that $\mathrm{E}$ regulation may help explain the differential reactivity often observed between men and women. Men tend show a higher cortisol increase than women when performing a psychosocial stress task (Kajantie \& Phillips, 2006); however, results are mixed when physical stressors and pharmacological challenges are employed (Kirschbaum, Wust, \& Hellhammer, 1992; Roca et al., 2005; Uhart, Chong, Oswald, Lin, \& Wand, 2006). Another consideration is that HPA-axis 


\section{Transdisciplinary Allostatic Load Review}

activities generally increase with ageing, sometimes so much so that this effect can be three times stronger in postmenopausal women compared to older men (Otte et al., 2005). Furthermore during the menstrual cycle, E and progesterone vary greatly and the use of a hormone-based contraceptive pill or menopause-related hormone therapy can impact stress reactivity (Bouma, Riese, Ormel, Verhulst, \& Oldehinkel, 2009; Kajantie \& Phillips, 2006; Kirschbaum, Kudielka, Gaab, Schommer, \& Hellhammer, 1999; Kirschbaum, Pirke, \& Hellhammer, 1995; Komesaroff, Esler, \& Sudhir, 1999; Marinari, Leshner, \& Doyle, 1976; Patacchioli et al., 2006; Prinz, Bailey, Moe, Wilkinson, \& Scanlan, 2001). While evidence is scarce, particularly concerning progesterone, it does seem that $\mathrm{E}$ has beneficial effects on dampening the HPA-axis, although much more research is needed before drawing any conclusions (Kajantie \& Phillips, 2006).

Differential stress responses observed between sexes and at different hormonal states might depend on the nature of the stressor. One explanation is that each stressor has a different route that triggers the HPA-axis. Psychosocial stressors involve the limbic system since it is based on the conscious interpretation of the situation whereas physical stressors trigger the hypothalamus, while pharmacological challenges can impact all three levels of the HPA-axis depending on the agent used (Dedovic, Duchesne, Andrews, Engert, \& Pruessner, 2009).

Interestingly, brain structures involved in the interpretation of a stressor and regulation of the HPA-axis are also rich in E receptors that are responsive to different $\mathrm{E}$ levels and are sexdimorphic in terms of developmental programming. For example, the hippocampus is involved in the appraisal and contextualization of stress, is essential when sorting memories under stress, and is structurally sensitive to the neurotoxic effects of cortisol. As E have neuroprotective effects, hormone therapy (HT) following menopause can protect the integrity of the hippocampus in a duration- and potentially time-dependent manner.

It has been observed that women using HT have larger HVs than non-users and men 


\section{Transdisciplinary Allostatic Load Review}

(Eberling et al., 2003; Lord, Buss, Lupien, \& Pruessner, 2008). This sexual dimorphism suggests that sex and/or gender and sex steroids may have a significant impact on HPA-axis regulation and AL levels as one ages (for a review, see (Wolf \& Kudielka, 2008). In order to resolve this issue and hopefully develop individualized approaches to health prevention and care, studies investigating multiple levels of outcomes that interact with the aforementioned variables are needed.

Like $\mathrm{T}$, the primary immunological mediators of $\mathrm{AL}$ are particularity sensitive to $\mathrm{E}$ regulation. E play a role in the sex difference characterizing many conditions with inflammatory components including stroke, bone metabolism, Alzheimer's and Parkinson's diseases, and even chronic autoimmune diseases such as multiple sclerosis and rheumatoid arthritis. Whether E have protective anti-inflammatory effects or pro-inflammatory properties is uncertain. Indeed, translating animal and in-vitro results on modulatory effects of $\mathrm{E}$ on cytokines to the human condition is particularly difficult and complex since the site of action (cells, organs), E dosage administered, compound studied (17 $\beta$-estradiol vs. conjugated E), sex, menstrual cycle, pregnancy, menopausal status, exogenous administration (i.e., contraceptive pill, HT), and age all have significant impacts on the effects and interactions observed (Cutolo, Capellino, \& Straub, 2008; Cutolo \& Straub, 2009; Cutolo et al., 2004; Hughes \& Clark, 2007; Pfeilschifter, Koditz, Pfohl, \& Schatz, 2002; Rossouw et al., 2008). Interestingly, evidence suggests that a timedependent modulatory effect of $\mathrm{E}$ on cytokines occurs with increased risk of CVD around menopause and hot flashes and accelerated phase of bone loss being limited to the first 5-10 years following menopause (Kronenberg, 1990; Maxwell, 1998; Pfeilschifter et al., 2002). Other pathways accounting for sex differences in several AL-related conditions involve differential lipidic and glycemic functions suggesting that E exert a lifetime modulatory role on related pathologies (e.g., diabetes, obesity). 


\section{Transdisciplinary Allostatic Load Review}

E levels are notoriously linked to cardiovascular risk at early as well as old age. Sex hormones appear to modulate lipid levels, especially HDL-cholesterol and insulin resistance in pre-pubescent children and adolescents (Agirbasli et al., 2010). Moreover, E have beneficial effects on lipid metabolism by decreasing low-density lipoprotein (LDL)-cholesterol and increasing HDL cholesterol. HT studies that examine postmenopausal women converges towards a protective role of E against CVD (O'Brien \& Nguyen, 1997). However, randomized controlled trials such as the Heart and Estrogen/Progestin Replacement Study (HERS) and the Women's Health Initiative (WHI) study led to unexpected and puzzling results: women with coronary heart disease (CHD) in the hormone group had a higher risk of CHD events during the first year followed by a decrease during year 3 to 5 and no effect after nearly 7 years (Grady et al., 2002).

The WHI results have been widely interpreted as demonstrating that combined HT has no beneficial effect and may even increase CVD. These controversial results were in disagreement with results from large epidemiological studies that showed protection by HT. This led researchers to believe that women in the observational studies must have initiated HT at or near the menopausal transition. Indeed, treatment in the WHI studies started 12 years after menopause, such that beneficial effects of E could only be observed if treatment was initiated during the menopausal transition before damages linked to E deficiency were present and potentially irreversible, therefore rendering HT more harmful than beneficial in later life (Harman et al., 2005a; Harman, Naftolin, Brinton, \& Judelson, 2005b; Low et al., 2002; Miller et al., 2009; Rossouw et al., 2007). It is important that future research address this controversy.

$\mathrm{E}$ are also involved in insulin regulation and glucose metabolism, although mixed results prevent any conclusions. Both endogenous and exogenous $\mathrm{E}$ are known to influence insulin sensitivity across the menstrual cycle, during pregnancy, in the menopausal transition, and in relation to hormonal contraception and HT (Bruns \& Kemnitz, 2004). Menopause, in particular, 


\section{Transdisciplinary Allostatic Load Review}

effects body composition in some women, which in turn is associated with impairments in glucose metabolism and insulin sensitivity. Whether E effects on glycemic regulation depend on body composition changes and/or age is unknown (Bruns \& Kemnitz, 2004; Szmuilowicz, Stuenkel, \& Seely, 2009).

Several studies have documented E effects on cognitive functions (e.g., learning and memory), on mood states, as well as on neurodevelopmental and neurodegenerative conditions. E have neuroprotective properties ranging from decreased neuronal loss, neurogenesis, neurotransmission, synaptic plasticity and dendritic spine density and connectivity (Behl, 2002; McEwen, 2002a). Moreover, E may impact brain metabolic states by enhancing glucose transport and cerebral blood flow, which are markedly altered in Alzheimer's disease (Eberling, Reed, Coleman, \& Jagust, 2000; Greene, 2000; Maki \& Resnick, 2000). Cognitive functions are indeed sensitive to the effects of $\mathrm{E}$, as differences are found during the menstrual cycle, pregnancy, menopausal transition, and upon use of HT later in life (Ancelin \& Ritchie, 2005; Sherwin, 2007). Timing of HT initiation following menopause and duration of treatment may also be important factors to consider when delineating effects of estrogens on cognitive functions (MacLennan et al., 2006).

Increased endogenous $\mathrm{E}$ exposure appear to decrease the risk of $\mathrm{AD}$, with later age at menarche increasing the risk of developing AD later in life (Paganini-Hill \& Henderson, 1994). Interestingly, females carrying the $\mathrm{E} 4$ allele of the ApoE gene with longer reproductive periods are at even greater risk of dementia (Geerlings et al., 2001). Furthermore, women who undergo menopause surgically are at increased risk of cognitive impairment or dementia, the risk of which increases the earlier the surgery was done (Rocca et al., 2007). Several studies have even found that reproductive events in a woman's life, such as age of menarche, the postpartum period, and menopause can increase the risk of developing psychiatric conditions (Bennett, Einarson, Taddio, 


\section{Transdisciplinary Allostatic Load Review}

Koren, \& Einarson, 2004; Brandes, Soares, \& Cohen, 2004; Pigott, 2003; Ross \& McLean, 2006;

Steiner, Dunn, \& Born, 2003). For instance, an analyses of the NHANES cohort found that earlier age of menarche was associated with increased AL levels (Allsworth, Weitzen, \& Boardman, 2005). As women have heightened vulnerability for developing specific mood disorders like depression, it will be important that future AL studies take female sex hormones into consideration. This will help reveal the degree to which female sex hormones interact with other processes to influence key biological and psychological outcomes.

\section{Conclusion and future directions}

These results collectively suggest that AL trajectories might be effected by differences stemming from sex, gender, and lifelong interactions to sex steroid exposures. It is hypothesized that if gender inequalities could be decreased or even eliminated, sex differences would no longer exist in these conditions (Lips, 2008). To better understand sex differences in AL, special attention must be directed toward the differential socio-cultural and biological contexts of individuals. An important challenge will also be to understand the dynamic interactions among sex and gender differences in psychopathologies throughout lifespan development that change throughout history. For instance, cardiovascular disease, once a predominantly male pathology, now claims at least $41 \%$ of all deaths of Canadian women in comparison to $37 \%$ for men (Plotnikoff, 1997). This could be partially explained by the fact that women have increased their participation in the workforce by $126 \%$ over the last 30 years (World-Bank, 2001) and now represent $42 \%$ of the entire global labor force (ILO, 2000; WHO, 1999). Perhaps this equitable progress has had a physiological price. Higher levels of stress for working women who must balance multiple workfamily roles and responsibilities can indeed further strain their health and well-being (Lagerlof, 2005). By applying a transdisciplinary approach that elucidates how sex, gender, and sex 


\section{Transdisciplinary Allostatic Load Review}

hormones interact within the context of society as a whole, we will be adding an important piece to the puzzle addressing AL sex differences.

In the first part of this review article, we have explored how early life adversities, genetics, environmental toxins, and sex differences can influence AL levels throughout lifespan development. Now in the second part, models of AL will be discussed for three psychological circumstances; namely, severe mental illnesses like schizophrenia and bipolar disorder, the dementia spectrum, and caregiving. These three experiences represent unique "experiments of nature" whereby AL manifests itself respectively by means of pharmacological remediation, pathological aging, or by virtue of helping others at personal detriment. Beginning with severe mental illness, we shall elucidate potential mechanisms whereby healing the distressed mind can be hurtful for the body.

\section{Schizophrenia and Bipolar Disorder}

The AL index could be particularly powerful in identifying schizophrenic or bipolar patients at high-risk of developing serious co-morbidities due in part to pharmacology and behavioral compensation. As this section will elaborate, we believe that the AL index could be a useful monitoring tool to facilitate and implement preventive care in these severe mental illnesses (SMI). The AL index could additionally be used to assess long-term side-effects of pharmacological treatment and complex intervention programs, as no tool to achieve this goal is currently available. We first review physiological correlates of schizophrenia and bipolar disorder within an AL framework, followed by pharmacological reversals of these dysregulations, and then conclude with how pharmacology can provoke co-morbidities.

Physiological dysregulations in severe mental illness 


\section{Transdisciplinary Allostatic Load Review}

Individuals with SMI are at increased risk of physiological dysreguations. No studies have assessed AL indices vis-à-vis schizophrenic and bipolar patients (for an excellent theoretical review, see Kapczinski et al., 2008); however, a review of individual biomarkers suggests that it could be a useful and easily constructed measure of multi-systemic functioning throughout the disease courses. In considering primary mediators of AL, increased basal HPA-axis activity can be found across different pathological stages in schizophrenia and bipolar disorder (Gallagher, Watson, Smith, Young, \& Ferrier, 2007; Linkowski et al., 1994). Across the board, higher baseline cortisol levels have been reported in drug-naive first-episode patients with schizophrenia (Muck-Seler et al., 2004; Walsh, Spelman, Sharifi, \& Thakore, 2005) as well as in medicated (Gallagher et al., 2007; Ritsner et al., 2007) and in chronic schizophrenic patients (Yilmaz et al., 2007).

In contrast to basal activity, findings focused on HPA reactivity assessed by the dexamethsone suppression test in schizophrenic patients are less consistent: both increased (Muck-Seler, Pivac, Jakovljevic, \& Brzovic, 1999) and decreased reactivity (Ismail, Murray, Wheeler, \& O'Keane, 1998) are reported. Conversely, in bipolar patients, elevated reactivity has been found (Sher, 2006) regardless of the phase of the illness (Watson, Gallagher, Ritchie, Ferrier, \& Young, 2004). Interestingly, the persistence of abnormal HPA-axis reactivity has also been observed in a portion of patients in remission who are at higher risk of depressive relapse (Vieta et al., 1997).

One potential pathophysiological pathway linking cortisol release to schizophrenic symptoms is via its interference with dopamine functioning prominently implicated in psychotic symptoms. Cortisol secretion augments dopamine neurotransmission (Czyrak, Mackowiak, Chocyk, Fijal, \& Wedzony, 2003; Dallman et al., 2004; Marinelli, Rudick, Hu, \& White, 2006; Moghaddam, 2002; Pruessner, Champagne, Meaney, \& Dagher, 2004) and the HPA-axis and 


\section{Transdisciplinary Allostatic Load Review}

dopaminergic neurocircuitry appear to reciprocally activate each other (Walker, Mittal, \& Tessner, 2008). Consistently, higher cortisol responses to amphetamines are related to more positive subjective drug effects, higher ratings of positive drug effects, and greater dopamine release in normal individuals (Oswald et al., 2005).

DHEA represents another primary mediator of AL that acts as a functional HPA-axis antagonist (Maninger, Wolkowitz, Reus, Epel, \& Mellon, 2009). The DHEA-to-cortisol ratio in chronic schizophrenia patients is negatively correlated with the duration of illness (Maninger et al., 2009). Moreover, decreased DHEA levels are associated with the severity of disease and cognitive impairment while medication might normalize levels. In the bipolar population, research is scarce, with only one report of normal DHEA levels (Gallagher et al., 2007). In sum, abnormalities in HPA-axis functioning in SMI patients seem to be characterized by increased basal cortisol levels, abnormal diurnal patterns of secretion, and dampened GC receptor signaling.

Different cytokines also function as complex primary mediators that contribute to AL (Kapczinski et al., 2008). This is due in part to their interactions with the neuroendocrine system and involvement in mood regulation. For example, interleukin-2 (IL-2) can elicit positive and negative symptoms of schizophrenia in normal individuals, including hallucinations, distortions, impaired cognition, and fatigue (Denicoff et al., 1987).

Individuals with schizophrenia have increased blood levels of IL-6, interleukin 1 receptor agonist (IL-1RA), soluble interleukin 2 receptor (sIL-2R) and decreased secretion of IL-2 by peripheral blood leukocytes (Potvin et al., 2008). In bipolar patients, elevations in IL1 receptor (IL1-R), IL-2R, IL-4, IL-6, IL-8, and a decrease in IL-2 have been identified (Breunis et al., 2003; Kim, Jung, Myint, Kim, \& Park, 2007; Liu et al., 2004; O'Brien, Scully, Scott, \& Dinan, 2006; Ortiz-Dominguez et al., 2007; Tsai et al., 1999). Based on these results, an ongoing "proinflammatory syndrome" in both schizophrenic and bipolar patients may manifest itself. However, 


\section{Transdisciplinary Allostatic Load Review}

it is difficult to distinguish between the relative influence of pathophysiological mechanisms inherent to SMI and other factors that influence cytokines levels, such as adiposity and, as will be discussed shortly, interactions with medications and associated biomarkers (Bob et al., 2010; Trayhurn \& Wood, 2004).

Pharmacotherapeutic factors in severe mental illness

In the vast majority of cases, the treatment of schizophrenia and bipolar disorder relies on acute pharmacological interventions aimed at overt psychotic/affective symptoms. These drugs have considerable potential to modulate ongoing allostatic processes (e.g., HPA-axis reactivity, immune regulation, neuroplasticity, cognitive functions, metabolic regulations) by antagonizing some while synergizing others.

The following will discuss commonly established pharmacological classes of drugs, namely first-generation antipsychotics (FGA or typical), second-generation antipsychotics (SGA or atypical), mood stabilizing agents and different classes of antidepressants used in the treatment of SMI. SGIs dampen over-activation of the stress axis by reducing ACTH and cortisol levels in schizophrenic patients (Mann et al., 2006; Markianos, Hatzimanolis, \& Lykouras, 1999; Ryan, Sharifi, Condren, \& Thakore, 2004; Zhang, Zhou, Cao, Wu, \& Shen, 2005) as well as in healthy individuals (Cohrs et al., 2006). Higher pretreatment cortisol levels have been related to higher antipsychotic responsiveness (Ritsner et al., 2005) and cortisol levels correlate with negative symptoms following withdrawal from SGAs (Zhang et al., 2005).

On the other hand, conflicting results exist regarding the effect of mood stabilizing agents such as lithium and carbamazepine on HPA-axis responsivity (Bschor et al., 2002; Watson et al., 2004). Antidepressant drugs impact HPA-axis activation via diverse mechanisms of action (Horstmann et al., 2009; Schule, 2007). Interestingly, the potential of selective serotonin reuptake 


\section{Transdisciplinary Allostatic Load Review}

inhibitors (SSRIs) to restore disturbed HPA-axis feedback control could be beneficial not only for patients with affective disorders (Walker et al., 2008) but also adolescents at clinical risk for psychosis. Indeed, some SSRIs appear to attenuate prodromal psychotic symptoms and reduce the risk of converting to axis I psychosis (Cornblatt et al., 2007).

In addition, psychotropic drugs have important immunomodulatory effects. FGAs like haloperidol, chlorpromazine, and flupentixol inhibit certain aspects of lymphocyte proliferation in vitro (Baker, Santalo, \& Blumenstein, 1977; Schleuning, Duggan, \& Reem, 1989). In vivo data also indicate that antipsychotic medication may be responsible for the increases in IL-2R and decreases of IL-2 (Potvin et al., 2008; Zhang et al., 2009). These findings suggest that antipsychotic administration may have immunosuppressive effects (Maes, Meltzer, \& Bosmans, 1994).

The potential to modulate neuroendocrine and immune regulation illustrates that the effects of psychiatric medications currently in vogue extend far beyond tuning doses in an isolated manner, one neurochemical system at a time. Rather, psychotropic agents can promote neuroprotective and neurotrophic processes involving multiple signaling pathways (Lieberman et al., 2008). For instance, patients treated with antipychotics have reduced oxidative stress (Dakhale, Khanzode, Saoji, Khobragade, \& Turankar, 2004), lower levels of lipid peroxidation (Kropp et al., 2005), and specific SGAs might even be able to ameliorate gray matter loses in patients in the early stages (Lieberman et al., 2005; van Haren et al., 2007).

Likewise, mood stabilizing and antidepressant treatments have also been consistently reported to influence neuroplastic processes (Hunsberger, Austin, Henter, \& Chen, 2009). Neuroimaging studies in clinical populations reveal that lithium treatment is associated with an increase of grey matter in different limbic regions such as the anterior cingulate (Bearden et al., 2007), hippocampus (Yucel et al., 2007), amygdala (Foland et al., 2008), as well as with regional 


\section{Transdisciplinary Allostatic Load Review}

increases in N-acetyl aspartate (NAA), an index of neuronal viability (Moore, Bebchuk, Wilds, Chen, \& Manji, 2000). Chronic administration of antidepressant agents have also been shown to increase hippocampal neurogenesis in animal models and prevent adverse effects of chronic stress exposure on the brain in the animal model (Hunsberger et al., 2009). In humans, antidepressant treatment may also increase BDNF levels in depressed patients (Shimizu et al., 2003).

Consistently, the neuroplastic potential of psychiatric medications appears to be paralleled by clinically relevant cognitive improvements. A number of clinical and multicenter trials have reported that SGAs improve various cognitive symptoms in schizophrenic patients (Cornblatt et al., 2002; Harvey et al., 2004; Potkin, Fleming, Jin, \& Gulasekaram, 2001; Schuepbach, Keshavan, Kmiec, \& Sweeney, 2002; Stip et al., 2003), but effects are less pronounced using FGAs (Bilder et al., 2002; Green et al., 2002). These findings indicate that pharmacological interventions may decelerate, halt, and even reverse the detrimental cognitive processes found in SMI. Unfortunately, where psychiatry can positively take away, it can also negatively give in the form of diverse side-effects that can manifest themselves as secondary and tertiary outcomes. Such pharmaceutically induced conditions contribute to distressing rates of co-morbidities that exacerbate AL levels further in SMI patients.

Antipsychotics are associated with an increased risk of developing the metabolic syndrome via weight gain, abnormalities in lipids, and glucose metabolism. Antipsychotic agents differ significantly in their potential to cause clinically relevant weight changes ( $>7 \%$ increase)(Haddad \& Sharma, 2007). There are several mechanisms proposed to explain the weight gain related to the antipsychotic treatment. Firstly, a common side-effect of antipsychotics is increased appetite (Kroeze et al., 2003; Muller, Muglia, Fortune, \& Kennedy, 2004) due to genetic mechanisms (Ellingrod et al., 2005) and impaired molecular actions (Allison et al., 1999; Elman, Borsook, \& Lukas, 2006). Secondly, due to the their sedative effects, antipsychotics contribute to decreased 


\section{Transdisciplinary Allostatic Load Review}

physical activity and energy expenditure (Lublin, Eberhard, \& Levander, 2005). Last, SGAs can further deteriorate pre-existing hedonic interferences with the opioidergic system in schizophrenic patients (Elman et al., 2006).

On the other hand, no weight gain is observed in about $25 \%$ of cases of metabolic syndrome under antipsychotic treatment, suggesting that other antipsychotic-induced metabolic disturbances might be at play (Newcomer, 2005). In a Finnish cohort study (Saari et al., 2004), it was concluded that patients on antipsychotic medications were 3-times more likely to have elevated cholesterol and triglycerides profiles than non-medicated control subjects. In addition to lipid profiles, antipsychotics may directly affect pancreatic functions and induce glucose abnormalities (Haddad \& Sharma, 2007). Literature regarding adverse metabolic effects of mood stabilizers and antidepressants is currently less comprehensive. Nonetheless, treatment with major mood stabilizers like lithium (Vendsborg, Bech, \& Rafaelsen, 1976), valproate, and carbamazepine (Swann, 2001) are associated with weight gain. That pharmacotherapy can act as both antidote and venom represents the difficulties of altering the biological functioning of selected systems without contaminating others. The reader will remember that these effects are a central criticism addressed by the allostasis concept that cautions against the dangers of iatrogenically inducing illness in patients.

\section{Iatrogenic effects and pharmaceutical allostatic load}

In addition to negative physiological effects, several psychotropic agents seem to interact with illness-related predispositions to synergistically worsen some unhealthy lifestyle behaviors and ultimately induce a "pharmacological" AL. We define pharmaceutical AL (PAL) as the adverse iatrogenic ("brought on by the healer") effects medications can exert that then inadvertently prompt individuals to remediate the biological system(s) effected by unhealthy 


\section{Transdisciplinary Allostatic Load Review}

means (e.g., smoking (see Figure 5), substance abuse). Current evidence points to three critical neurochemical systems that influence PAL: the (1) acetylcholinergic, (2) dopaminergic, and (3) opioidergic systems.

Firstly, activity of cholinergic circuitry (e.g., nicotinic and muscarinic receptors) is related to memory and attention capabilities. Many psychotropic agents are known to antagonize muscarinic receptors (Minzenberg, Poole, Benton, \& Vinogradov, 2004) that can inadvertently induce cognitive perturbations for countless schizophrenic patients (Minzenberg et al., 2004). Patients are therefore very likely to engage in behaviors that temporarily re-regulate these impairing symptoms. Such iatrogenic cognitive impairments only add to the already pre-existing perturbations of the cholinergic system found in schizophrenic patients. Importantly and unfortunately, nicotine can counteract these PAL induced cognitive dysfunctions (Levin, Wilson, Rose, \& McEvoy, 1996), which might explain why psychiatric patients extensively self-medicate when treated with anti-cholinergics.

Secondly, dopamine D2 receptor antagonists central to antipsychotic dampening of positive symptoms (i.e., delusions, hallucinations) due to excessive dopamine levels are also linked to well-known extrapyramidal (i.e., akathisia, dyskinesia, dystonia), endocrine (i.e., hyperprolactinemia), and a host of other side-effects. As evidenced by pharmacological studies in a healthy sample, haloperidol (a FGA and potent D2 receptor antagonist) administration causes psychomotor slowing and impaired executive function (Peretti et al., 1997) as well as sustained attention deficits and decreased self-reported quality of life (Saeedi, Remington, \& Christensen, 2006). This latter negative impact on affective regulation is consistent with reports of dysphoric responses occurring during antipsychotic treatment in psychiatric populations (Voruganti \& Awad, 2004). 


\section{Transdisciplinary Allostatic Load Review}

Differential potency of FGA and SGA to induce dopamine deficits seems to be clinically important, as illustrated by lower rates of substance abuse (Noordsy \& Green, 2003) in patients treated by clozapine (a SGA) compared to FGAs. Furthermore, D2 receptor blocking in the striatum might increase risk of addictive behaviors (Krystal et al., 2006). Pharmacological D2receptor modulation worsens pre-existing vulnerabilities towards increased tobacco smoking in psychiatric patients. In a prospective study, the potent D2-receptor blocking FGAs were associated with an increase in nicotine dependence, but not in SGAs (Kim, Han, Joo, \& Min, 2010). Interestingly, treatment with aripiprazol, a partial agonist of the D2 receptor, reduces both nicotine dependence and cigarette cravings (Kim et al., 2010). Similarly, patients treated with clozapine display lower rates of smoking and higher rates of smoking cessation compared to those treated with FGA (McEvoy et al., 1995). It is noteworthy that nicotine has the potential to alleviate several physical side-effects related to D2 receptor blocking, such as akathisia (Yang, Nelson, Kamaraju, Wilson, \& McEvoy, 2002), a syndrome of unpleasant restlessness. Consistently, schizophrenic patients self-report higher desires for "sensorimotor manipulation", "need for stimulation" and score lower on "sociability" than normals (Barr, Procyshyn, Hui, Johnson, \& Honer, 2008), suggesting that their motivations towards maladaptive behaviors represents PAL related to antipsychotic medications.

Thirdly, SGAs may increase opioidergic neurotransmission and subsequently alter the "liking" processes of reward systems, leading to hedonic preference for sweet and fatty foods (Elman et al., 2006). Once again, these inclinations toward diets that counteract the effects of medications contribute to increased rates of obesity and metabolic problems. While we must be cautious in deriving any conclusions given differences among specific agents, dosage regiments, and an overall paucity of evidence, it can nevertheless be postulated that interactions between psychotropic drugs and several important neurobiological mechanisms might drive PAL effects. 


\section{Transdisciplinary Allostatic Load Review}

At the heart of these three neurochemical systems involved in PAL are interactions to regulate HPA-axis activity by dampening cortisol dynamics associated with SMI and medications.

In sum, pharmacological agents have neuroprotective and neurotrophic effects, but they can also aggravate pre-existing susceptibilities by PAL, which in turn contribute further to dysregulation of primary mediators, secondary outcomes, and co-morbid tertiary AL outcomes (e.g., substance abuse, cardiovascular disease, somatic complaints; see Figure 6).

\section{Conclusion and future directions}

The concept of PAL is formulated here in the hopes of contributing greater understanding to the complexities of pharmacological remediation. That pharmacotherapy can act as a form of $\mathrm{AL}$ in interaction with behavioral compensation is an idea that could be explored further in line with current thinking coming from research on addiction (e.g., (Koob, 2008, 2009; Koob \& Le Moal, 2008a, 2008b). In so doing, we do not wish to minimize the important achievements of psychiatry and pharmacotherapy in general. On the contrary, the aims of this section is to add extra pieces of knowledge to apply the AL model within the realm of clinical practice so as to refine our definitions of health and disease. Importantly, delineating the interactions among SMIs, psychopharmacology, and differential clusters of symptomatologies provides insights into the mechanisms and heterogeneity in disease courses so central to developmental psychopathology.

Within the current paradigms surrounding SMI (as well as in other fields), research priorities, nosological classifications, treatment strategies, and social policies are illness-centered. As useful as these approaches are in identifying biomolecular correlates and vulnerability factors for SMI, they are often not incorporated within patient-centered approaches by focusing on pathology alone. The biggest caveat of this pathology-driven approach is that it misses out on the immense potential to promote resiliency factors associated with positive health in individuals 


\section{Transdisciplinary Allostatic Load Review}

suffering from SMI. A positive consequence of embodying a transdisciplinary perspective is therefore to recognize the multiple levels (e.g., psychosocial, behavioral, genetic) of AL and to foster patients' sense of competence and engagement in their own treatment.

The concept of top-down regulation of cognitive, autonomic and neuroendocrine function inherent to the AL model will provide a rationale to support emerging trends that reorient therapeutic strategies and emphasize the role of healthy lifestyle promotion, social support, and governmental policies (McEwen, 2008). Basing interventions on a partnership rather than a model where the patient is a passive consumer of healthcare will further encourage patients' coping abilities, dignity, and the person taken as a whole within his/her environmental context (Mezzich, 2010).

Because SMIs like bipolar disorder and schizophrenia increase AL, elucidating how key biomarkers are linked to symptomatologies - before and after interventions - necessitates personcentered approaches that appreciate, for instance, gene-environment interactions or differential neurobiological development throughout the life cycle. In delineating these different trajectories, it is essential that we further identify specific biopsychosocial signatures of specific disease vulnerabilities in relation to AL. In this spirit, where dementia falls on the continuum ranging from normal to abnormal aging represents another research area to explore. The following section will review various sub-sets of dementia as pathways towards AL.

\section{Dementia(s): A Question of Biomarkers?}

Dementia is a spectrum whereby the most prevalent form is Alzheimer's disease (AD). Those afflicted experience gradual degeneration of memory and other cognitive faculties (McKhann et al., 1984) presumably due to $\beta$-amyloid plaque accumulations and neurofribrillary tangles. In other cases, CVDs like stroke can cause vascular lesions in the brain that can result in 


\section{Transdisciplinary Allostatic Load Review}

the manifestation of cognitive deficits found in vascular dementia $(\mathrm{VaD})$ or mixed dementia when concurrent in $\mathrm{AD}$ patients. At a preclinical stage of dementia are individuals with mild cognitive impairment (MCI) that present with subjective cognitive complaints, corroborated neuropsychological deficits, but no functional or social deficits (Petersen et al., 1999). Of particular interest are the physiological dysregulations characterizing distinct conditions that typify normal from abnormal aging.

\section{Physiological dysregulations in dementia}

While no studies to date have assessed AL vis-à-vis dementia, the cognitive declines found in the MacArthur cohort (Seeman et al., 2001; Seeman et al., 1997b) imply that it could be a useful tool for detecting biological signatures of certain forms of dementia over others. For instance, multisystemic dysregulations captured by the AL index are strongly linked to frailty (Fried et al., 2001; Gruenewald, Seeman, Karlamangla, \& Sarkisian, 2009; Szanton, Allen, Seplaki, Bandeen-Roche, \& Fried, 2009). It would be beneficial that future research investigate how primary mediators and secondary outcomes might lead to differential susceptibilities.

The role of stress hormones in the etiology of dementia is controversial (see Table 3). While some research suggests that AD patients are hypersecretors of cortisol (Giubilei et al., 2001), longitudinal studies are needed to solidify the causal direction of this association. For example, increased urinary noradrenalin levels correlate with cognitive decline in healthy elderly men (Karlamangla, Singer, Greendale, \& Seeman, 2005b) and increased cortisol secretion in normal elderly are associated with cognitive declines in some studies (Greendale, KritzSilverstein, Seeman, \& Barrett-Connor, 2000; Karlamangla et al., 2005a; Lee et al., 2007a; Li et al., 2006; Lupien et al., 1994; Seeman, McEwen, Singer, Albert, \& Rowe, 1997b) but not all (Csernansky et al., 2006; Kalmijn et al., 1998; Peavy et al., 2009) 


\section{Transdisciplinary Allostatic Load Review}

Two studies (Csernansky et al., 2006; Swanwick et al., 1998) found that increased cortisol levels over the years were associated with cognitive decline in individuals with clinical dementia ratings of 0.5 that some consider to resemble MCI. Conversely, lower morning cortisol levels at baseline were associated with future cognitive decline in normal elderly who are E4 carriers for the ApoE gene (Gerritsen, Comijs, Deeg, Penninx, \& Geerlings, 2009) and higher cortisol is associated with slower cognitive decline in MCI (Peavy et al., 2009). Some studies found an association with cognitive decline later on in AD patients (Huang et al., 2009; Umegaki et al., 2000; Weiner, Vobach, Olsson, Svetlik, \& Risser, 1997), but not in individuals with CDR 1,0 or

more (Csernansky et al., 2006; Swanwick et al., 1998). Taken together, these findings suggest that high cortisol levels lead to differential risk-profiles depending on whether individuals are ApoE4 carriers, and whether they suffer from MCI or AD. They do not, however, provide sufficient evidence to make any conclusions regarding the role of stress hormones in dementia.

Turning to another primary mediator of AL, no studies have found associations between dehydroepiandrosterone sulfate (DHEA-S) and dementia incidence (Barrett-Connor \& Edelstein, 1994; Berr, Lafont, Debuire, Dartigues, \& Baulieu, 1996; Ponholzer et al., 2009), cognitive decline in normal elderly (Kalmijn et al., 1998), nor AD patients (Miller et al., 1998). DHEA-S may be predictive of general health declines and mortality for men (Barrett-Connor \& Edelstein, 1994; Berr et al., 1996).

Regarding cytokines (see Table 4), some studies have found that dementia incidence is associated with IL-6 (Engelhart et al., 2004), CRP (Schmidt et al., 2002; Xu, Zhou, Zhu, Fan, \& Liu, 2009), and TNF- $\alpha$ (Tarkowski, Andreasen, Tarkowski, \& Blennow, 2003); however, negative results have been found concerning CRP (Engelhart et al., 2004; Gallacher et al., 2010; van den Berg, Biessels, de Craen, Gussekloo, \& Westendorp, 2007) and IL-6 (Gallacher et al., 2010). Several studies have assessed the relations between baseline levels of inflammatory risk factors 


\section{Transdisciplinary Allostatic Load Review}

and cognitive decline. Notably, people with higher basal levels of IL-6 in the MacArthur cohort were at greater risk of cognitive decline measured at baseline, 2.5 and 7 years later (Weaver et al., 2002). Similarly, IL-6 was associated with cognitive decline for global measures of cognition (Alley, Crimmins, Karlamangla, Hu, \& Seeman, 2008; Jordanova, Stewart, Davies, Sherwood, \& Prince, 2007) or memory (Schram et al., 2007).

Amidst the breadth of studies conducted using CRP, most have failed to find an association with global cognitive decline (Alley et al., 2008; Dik et al., 2005; Holmes et al., 2009; Hoth et al., 2008; Jordanova et al., 2007; Laurin, David Curb, Masaki, White, \& Launer, 2009; Marioni et al., 2009; Schram et al., 2007; Yaffe et al., 2003). Exceptionally, increased CRP levels have been correlated with declines in executive functioning (Hoth et al., 2008) as well as in reasoning and memory abilities (Marioni et al., 2009). Using TNF- $\alpha$, higher levels were not related to global cognitive decline in older individuals (Yaffe et al., 2003) but rather in AD patients (Holmes et al., 2009). Clearly, more research is needed in order to ascertain the association between dementia and/or cognitive decline vis-à-vis inflammation. Note that the most important confounder in these studies was the variability in the measure of cognitive declines.

Several prospective studies have examined the metabolic syndrome (MetS) using the National Cholesterol Education Program (NCEP) criteria (Grundy, Brewer, Cleeman, Smith, \& Lenfant, 2004) as predictors of dementia (see Table 5). Overall, results here too are mixed, with some positive (Muller et al., 2007; Solfrizzi et al., 2009; Solfrizzi et al., In Press) and negative (Forti et al., 2010; Raffaitin et al., 2009) findings linking MetS to dementia susceptibility. It appears that MetS is more consistently associated with cognitive decline (Ho, Niti, Yap, Kua, \& Ng, 2008; van den Berg et al., 2007; Yaffe et al., 2007; Yaffe et al., 2004; Yaffe, Weston, Blackwell, \& Krueger, 2009) than with clinical dementia. Other prospective studies have focused only on cardiovascular biomarkers of MetS, showing that vascular indices are linked to dementia 


\section{Transdisciplinary Allostatic Load Review}

(Kalmijn et al., 1998; Kivipelto et al., 2006; Whitmer, Sidney, Selby, Johnston, \& Yaffe, 2005) but not cognitive decline (Mielke et al., 2007; Xiong, Plassman, Helms, \& Steffens, 2006).

Notably, two studies have found that MetS is protective in individuals ages 75 and up against cognitive decline (van den Berg et al., 2007) and dementia (Forti et al., 2010). This may reflect the paradox effect, where the risk for dementia in relation to some cardiovascular risk factors seems to be inverted when measured in midlife compared to old age. Similar conclusions were made in a review by Schnaider and colleagues (2004) whereby hypertension and hyperlipidemia in midlife but not in late life were associated with increased risk of dementia (Schnaider Beeri et al., 2004). Regardless of age, diabetes was consistently associated with increased risk of dementia . These age-dependent associations might explain previous inconsistent results.

Another point for future investigations to consider is which biomarkers and outcomes to use. Studies demarcating dementia risk factors have found that MetS or vascular indices are predictors of $\mathrm{VaD}$ but not $\mathrm{AD}$ in elderly individuals (Kalmijn et al., 2000; Raffaitin et al., 2009; Solfrizzi et al., 2009). Yet, other studies suggest that cardiovascular risk factors might also be independently associated with AD. For instance, hypertension in midlife may (Kivipelto et al., 2001) or may not (Luchsinger et al., 2005; Posner et al., 2002) be associated with AD. Skoog et al. (1996) found that hypertention at an older age is associated with AD but those who developed AD also showed bigger decline over the years previous to diagnosis (Skoog et al., 1996). Likewise, high total cholesterol is associated with AD when measured in midlife (Kivipelto et al., 2001) and old life (Notkola et al., 1998) but not in all studies (Li et al., 2005; Tan et al., 2003). Based on these inconsistencies, it can truly be said that biological signatures of dementia change as one ages and therefore cannot be considered finite. 


\section{Transdisciplinary Allostatic Load Review}

Consistent to this claim, one study found that total cholesterol levels of men with dementia - especially AD patients - declined 15 years prior to diagnosis and remained lower (Stewart, White, Xue, \& Launer, 2007). Results are mixed as well for diabetes in old age vis-à-vis AD (Luchsinger et al., 2005; Xu et al., 2009). Finally for obesity in AD, both positive associations in older women (Gustafson, Rothenberg, Blennow, Steen, \& Skoog, 2003) and negative associations (protective) in older individuals (Hughes, Borenstein, Schofield, Wu, \& Larson, 2009) have been found. In midlife, one study found that higher BMI was associated with future AD (Kivipelto et al., 2005), whereas baseline weight was not associated with AD in another study (Stewart et al., 2005). Noteworthy for the latter study was that men with AD lost significantly more weight over the last 6 years previous to the diagnosis than men without dementia, potentially representing a phenomenon referred to as wasting in gerontology.

In sum, the transitions from normal into abnormal aging and dementia appear to be associated with distinct physiological features that constantly change as one continues to develop into old age. Disagreement on the precise biological correlates of specific disease states informs us that much more research is needed using a developmental psychopathological perspective in alliance with transdisciplinarism. Concordantly, the advantage of a multisystemic measure like the AL index in combination with neuropsychological assessment and neuroimaging, might prove fruitful in this endeavor, perhaps even more so than teasing apart complex configurations of biomarkers. Turning now to neuroimaging, it is important to highlight upon inception how individual differences when examining peripheral markers of $\mathrm{AL}$ in the body are only more complicated when observing lifecourse changes in the brain.

Hippocampal volume in abnormal aging and dementia 


\section{Transdisciplinary Allostatic Load Review}

Because hippocampal atrophy is an infamous feature of $\mathrm{AD}$, much research has focused on how it corresponds to pathological aging. As a group, older adults generally have smaller HVs than young adults (Du et al., 2006; Resnick et al., 2000); however, there is substantial variation across age groups (Lupien et al., 2007). HV can predict cognitive decline among healthy older adults (Grundman et al., 2003; Jagust et al., 2006; O'Sullivan et al., 2009), therefore it might be a potential biomarker of pathological aging (Burgmans et al., 2009). We must be cautious, however, as it is difficult to ascertain whether HV is the chicken or egg in dementia. Among patients with $\mathrm{MCI}$ and $\mathrm{AD}$, for instance, the hippocampus is one of the first regions to show atrophy (Dickerson et al., 2001; Jack et al., 1999; Jack et al., 1997) and MCI patients with smaller HVs progress more rapidly to AD (Korf, Wahlund, Visser, \& Scheltens, 2004).

As an important brain region in regulating the HPA-axis, the hippocampus is particularly vulnerable to GC neurotoxicity associated with AL. For example, elevated chronic life stress over a 20-year period was associated with decreased grey matter volume in the hippocampus and orbitofrontal cortex (Gianaros et al., 2007) and older adults with increasing cortisol levels over six years exhibit hippocampal atrophy and poorer memory performance (Lupien et al., 1998). Taken together, these results indicate the long-term impact of cortisol dysregulations and AL, and their association with poor cognition and hippocampal atrophy (McEwen, 2007a, 2008), although they also underline the complexity of teasing apart disease pathways when dealing with interconnected biomarkers.

In addition to cortisol, chronic stress exposure increases the risk of developing insulin resistance and eventual type-II diabetes (Raikkonen, Keltikangas-Jarvinen, Adlercreutz, \& Hautanen, 1996) linked to reductions in HV (Convit, Wolf, Tarshish, \& de Leon, 2003), impaired memory performance (Convit, 2005; Gold et al., 2007; Hendrickx, McEwen, \& Ouderaa, 2005) and, most importantly, increased risk for later AD onset (Arvanitakis, Wilson, Bienias, Evans, \& 


\section{Transdisciplinary Allostatic Load Review}

Bennett, 2004; Lee et al., 1999; Schnaider Beeri et al., 2004). There is cross-talk among these systems, as overactive HPA-axis activity is common among patients with type-II diabetes (Bruehl et al., 2007; Lee et al., 1999). Another interconnected consideration is that depression among older adults is associated with increased cortisol levels and memory impairments (McAllister-Williams, Ferrier, \& Young, 1998), as well as increased risk for AD (Geerlings, den Heijer, Koudstaal, Hofman, \& Breteler, 2008; Ownby, Crocco, Acevedo, John, \& Loewenstein, 2006). Moreover, reductions in HV are found among older patients diagnosed with depression (O'Brien, Lloyd, McKeith, Gholkar, \& Ferrier, 2004; Steffens et al., 2000). Interestingly, evidence from elderly twins shows that genetics can only account for $40 \%$ of HV variance, whereas the other $60 \%$ are attributable to environmental factors that may exert an impact during different stages of one's lifespan (Sullivan, Pfefferbaum, Swan, \& Carmelli, 2001). These findings highlight the significance of factors related to the environment as well as AL for determining $\mathrm{HV}$ and functioning (McEwen, 2003c) and one's subsequent risk of dementia and/or cognitive decline.

Although the hippocampus is a sensitive brain region, it also has the capacity to adapt, protect, and renew itself against damage and AL via structural plasticity and neurogenesis in the dentate gyrus throughout adulthood (McEwen, 2001, 2002c). Indeed, a variety of pharmacological and behavioral factors have the capacity to protect the hippocampus. For example, physical exercise is associated with increased neurogenesis (Olson, Eadie, Ernst, \& Christie, 2006), larger HV and improved memory performance (Erickson et al., 2009), as well as enhanced executive functioning (Baker et al., 2010; Colcombe, Kramer, McAuley, Erickson, \& Scalf, 2004; Etgen et al., 2010; Kramer et al., 1999). Even under conditions of chronic stress, hippocampal atrophy and associated cognitive impairments can be reversed following the use of appropriate treatments

(Duman, Nakagawa, \& Malberg, 2001; Vermetten, Vythilingam, Southwick, Charney, \& Bremner, 2003; Vythilingam et al., 2004). Despite the vulnerability of the hippocampus to various 


\section{Transdisciplinary Allostatic Load Review}

toxic effects, it is remarkably resilient when modulated by factors that also contribute to reducing AL (McEwen, 2008; McEwen, de Leon, Lupien, \& Meaney, 1999). In this view, one can envision the brain itself as undergoing a form of allostasis via its plasticity under different circumstances.

\section{Conclusions and future directions}

Taken together, there is substantial evidence that biomarkers included in the AL model are associated with dementia, albeit we are far from knowing the exact biopsychsocial signatures of dementia's different faces. While this claim is based largely on MetS and vascular parameters of the AL index, several methodological considerations should be addressed given the diverse findings. Most importantly, the biomarkers incorporated in the AL construct depend largely on the participants' age and sex. For instance, some evidence suggests that cortisol and noradrenalin in men may be associated with future cognitive decline, while other studies indicate that cortisol might be protective in individuals with MCI. Secondly, the paucity of research using neuroendocrine and inflammatory biomarkers in relation to cognitive decline and neurotoxicity in relation to dementia limits any conclusions at this time. Finally, the prospective studies cited above all used different methodologies and inclusions of covariates (e.g., education, medication) that are not fully understood in relation to dementia. Furthermore, the role of neurological degeneration as in the case of hippocampal vulnerability should be explored further in transdisciplinary studies that incorporate AL biomarkers, brain imaging, and various other methodologies. Consistent with a developmental perspective, tracking whether certain clusters of vulnerabilities manifest themselves necessitate longitudinal investigations.

An important avenue for future research would be to demarcate in greater detail the sequence whereby AL mediators lead to primary effects that include oxidative stress (McEwen, 2008). Defined as a state of increased free radical production relative to antioxidant defenses, 


\section{Transdisciplinary Allostatic Load Review}

oxidative stress has been related to damage of several sub-cellular structures. For example, normal aging is associated with a slow progressive accumulation of oxidative damage (Wallace, 2005), but this phenomenon may be accelerated in brains of individuals suffering from neurodegenerative disorders (Mariani, Polidori, Cherubini, \& Mecocci, 2005). Disturbances in oxidative stress metabolism may therefore contribute to the development of secondary and tertiary outcomes of AL, representing a molecular primary effect. Another important question that remains to be addressed is whether hormonal signals (e.g., cortisol, DHEA) and inflammatory markers (e.g., IL6, CRP) influence global health outcomes when elevated in the context of existing systemic imbalances such as metabolic syndrome, pathological aging, or in the context of special behaviors and social situations. One elusive social situation that combines a special set of behaviors and psychological challenges is caregiving. Our final section will explore caregiving as a unique experiment of nature whereby individuals are at risk of increased AL by virtue of their altruism.

\section{The Stress and Strain of Caregiving}

Stress associated with caring for an elderly or mentally ill person can adversely affect the health status of caregivers. Although some caregivers are able to cope well with their role and experience few symptoms of distress along with a positive gain from the experience (Kramer, 1997; Schulz et al., 1997), growing bodies of literature document the stressful nature and health risks of being a caregiver. According to the National Alliance of Caregivers, an estimated 43 million Americans, over the age of twenty, function as informal caregivers providing unpaid care to older ill or disabled family member or friends as well as collectively saving the formal health

care system billions of dollars annually (Ory, Hoffman, Yee, Tennstedt, \& Schulz, 1999). Unfortunately, caregiving comes at a personal price as a chronic stressor (Bookwala \& Schulz, 2000; Vitaliano, 1993), thus representing a human model of chronic stress (Lupien et al., 2009). 


\section{Transdisciplinary Allostatic Load Review}

This section will correspondingly review the negative health outcomes of caregiving and studies that examine the psychological, physical, physiological, and social consequences of caregiving that are demonstrably related to increased AL.

\section{Psychological outcomes of caregiving}

Caregivers demonstrate higher levels of psychological distress as well as symptoms of depression and anxiety (Schulz, O'Brien, Bookwala, \& Fleissner, 1995; Schulz, Visintainer, \& Williamson, 1990). The inherently stressful nature of being a caregiver has been tested in several studies revealing the effects of AL. In a study on $\mathrm{AD}$ caregivers by von Känel and coworkers (2003), a greater number of negative life events related to increased AL levels based on more pronounced procoagulability responses to a 15-minute speech task was found. This unique finding suggests that high baseline AL (combined caregiving and life distress) is linked to a reduced ability for the caregivers to maintain hemostatic stability in response to a laboratory stressor (von Kanel, Dimsdale, Patterson, \& Grant, 2003). In another AL study mentioned earlier, the stress of providing care was tested in mothers of children with cancer and controls. Results showed that increased AL was associated with intensified post-traumatic stress disorder symptoms (Glover, 2006) and smaller right HV (Glover et al., 2008).

In addition to psychological effects, diminished cognitive function and general emotional distress also result from caring for a relative with a mental illness. In one of the few studies on caregiver stress and cognitive function, women who provided care to their ill spouses were found to have significantly increased risk of low cognitive functioning on several cognitive tests when compared to women who did not provide any care (Lee, Kawachi, \& Grodstein, 2004). The psychological experience of caregivers also examines caregivers' emotions and places their value system into question. For instance, self-conscious emotions (e.g., guilt and shame), general 


\section{Transdisciplinary Allostatic Load Review}

emotional distress (GED), and expressed emotion (EE) in family members of patients with schizophrenia revealed that increasing shame proneness was strongly associated with greater caregiver distress, whereas increasing guilt proneness was associated with less distress. The results suggest that caregivers of patients with schizophrenia who are shame prone may be predisposed to view incidents, such as having a relative with a mental illness, as something that reflects negatively upon themselves. Consequently, they may be more likely to experience high levels of the type of GED tapped by the Depression and Anxiety Stress Scale (de Mamani, 2010), shame, and social isolation.

Conversely, guilt proneness is associated with lower levels of GED. Guilt proneness may be associated with a certain level of interpersonal problem-solving in caregivers of schizophrenia patients that directly or indirectly lowers GED. For instance, guilt proneness may encourage relatives to make amends after being aggravated with a patient, or to try to assist an ill relative that one has recently shunned (de Mamani, 2010). This implies the potential therapeutic value of evaluating caregivers' guilt proneness to help identify individuals who may be at particularly high risk of experiencing psychiatric symptoms. Likewise, these findings suggest that clinicians should be cautious of attempting to lessen or eliminate caregivers' guilt that is associated with caring for a loved one with a mental disorder. Rather, assisting family members to find productive strategies to direct their guilt, such as providing support, advice, or other types of assistance to the patient, while also caring for their own needs may prove beneficial.

More recently, researchers have focused not only on providing care as a source of distress, but also on the caregiver's perception of how much the patient is suffering. Patient suffering is evident in three related and measureable ways: overt physical signs, including verbal and nonverbal expression of pain and physical discomfort, such as difficulty breathing; psychological symptoms of distress, such as depression and apathy; and existential or spiritual well-being, 


\section{Transdisciplinary Allostatic Load Review}

reflecting the extent to which religious or philosophical beliefs provide inner harmony, comfort and strength, or alternately, lead to despair (McClain, Rosenfeld, \& Breitbart, 2003; Schulz et al., 2007). Specifically, it has been shown that two types of patient suffering - emotional and existential distress - are significantly associated with caregiver depression and use of antidepressant medication (Schulz et al., 2008). Emerging evidence of this nature demonstrate the transdisciplinary assessment of factors such as the level of patient suffering, since they may contribute substantially.

\section{Physiological outcomes of caregiving}

Caregiver stress has been linked to adverse physiological health in a large number of studies. Specifically, elevated blood pressure (King, 1992; Moritz, 1992), heightened cardiovascular reactivity (Vitaliano, 1993), risk for coronary heart disease (Haley, Roth, Howard, \& Safford), elevated cortisol levels (de Vugt et al., 2005), lower immune function (Kiecolt-Glaser, Dura, Speicher, Trask, \& Glaser, 1991), and even increased mortality among spousal caregivers (Schulz \& Beach, 1999) have been observed.

With regards to the effects of chronic stress on GCs, one study in particular assessed caregivers who had elevated diurnal cortisol levels and tended to be older caregivers of patients with AD (de Vugt et al., 2005). Similar effects have been observed in a 7-day study of younger caregiving wives of patients with physical problems whereby caregivers had higher cortisol production when particular events related to caregiving occurred in comparison to controls (Davis et al., 2004). These findings provide evidence that psychological changes are indictors of chronic stress in caregivers and potentially brewing AL.

Further physiological effects of caregiving are apparent in a more recent study on the impact of caregiving strain and the risk for stroke and coronary heart disease among spousal 


\section{Transdisciplinary Allostatic Load Review}

caregivers. Results demonstrated that high caregiving strain was associated with higher estimated stroke risk with greatest effects for African American men providing care to their wives (Haley et al.). As well, a cohort study reported an almost twofold increase in risk of coronary heart disease in women who provide care to a disabled or ill spouse for 9 or more hours per week (Lee, Colditz, Berkman, \& Kawachi, 2003). Regardless of the caregivers' sex, the stress of providing care can be, in itself, a risk factor for stroke and coronary heart disease. It is important to note that women are often caregivers, and as such this represents a gender-role assignment that should be investigated further. Additionally, applying a developmental analysis that takes previous experiences in the lifespan into consideration could be helpful.

Ultimately, the immense stress and strain of caregiving impinges on caregivers' physiological functioning and increases their risk for physical health problems, leading even to premature mortality. In an edifying study, caregiving has been identified as an independent risk factor for death with increased risk in individuals experiencing caregiver strain over a 4-year period (Schulz \& Beach, 1999). Perceived stress has also been related to higher AL primary mediators in veteran dementia caregivers over two years in comparison to new and non-caregivers (Clark, Bond, \& Hecker, 2007). Collectively, these studies demonstrate that caregivers are at increased risk of mortality and ill health.

\section{Physical outcomes of caregiving}

The chronic stress of caregiving affects numerous dimensions of caregiver health, including self-reported health, health symptoms, illness and medication use (Asada, Kinoshita, \& Kakuma, 2000; Cacioppo et al., 1998; Schulz \& Beach, 1999; Vitaliano, Zhang, \& Scanlan, 2003; Zhang, Vitaliano, \& Lin, 2006). A revealing study has shown that caregivers experience a onethird increase in negative health symptoms after assuming caregiving responsibilities (Shanks- 


\section{Transdisciplinary Allostatic Load Review}

McElroy \& Strobino, 2001). Spousal caregivers also report an increase in days ill due to infectious disease, primarily upper respiratory tract infections (Esterling, Kiecolt-Glaser, \& Glaser, 1996). Results on caregiving stress and caregiver's functional ability indicate that caregivers who report higher levels of caregiving stress have poorer self-rated health, poorer physical function, more symptoms and high levels of depressed mood (Lu \& Wykle, 2007). Caregivers are also increased consumers of prescription medications (Schulz et al., 1997).

Self-care is often compromised as caregivers provide a high level of care for others. Common complaints include forgetting to take medications, having no time to exercise, nor see a doctor or rest when ill, and finally insomnia (Burton, Newsom, Schulz, Hirsch, \& German, 1997; Schulz et al., 1997). Several studies report sleep problems as a major adverse physical outcome of caregiving (Creese, Bedard, Brazil, \& Chambers, 2008; Flaskerud, Carter, \& Lee, 2000; Matsuda, Hasebe, Ikehara, Futatsuya, \& Akahane, 1997; McKibbin et al., 2005; Meltzer \& Mindell, 2006; Tsukasaki et al., 2006; Wilcox \& King, 1999). These problems include nighttime or early morning waking, difficulties in sleep onset, poor sleep quality, less slow-wave sleep, insomnia, and most commonly, sleep disruptions (Flaskerud et al., 2000; Matsuda et al., 1997; Tsukasaki et al., 2006; Wilcox \& King, 1999).

Sleep disruptions are a risk factor for poor mental health among caregivers and this has been well documented. Specifically, studies have reported that the quality of sleep is significantly associated with depression among caregivers of cancer patients as well as patients with AD (Carter \& Chang, 2000; Willette-Murphy, Todero, \& Yeaworth, 2006). In a similar vein, caregivers whose sleep was frequently disrupted were about 2.5 times more likely to suffer from poor mental health, compared to those whose sleep was not disrupted (Lam, 2008). Sleep is an essential and yet empirically unexplored component of the AL model (McEwen, 2006b) that should be explored in caregivers and other adverse conditions. Indeed, aberrations in sleep are 


\section{Transdisciplinary Allostatic Load Review}

associated to numerous biomarkers associated to secondary outcomes of AL (Copinschi et al., 2010; Knutson, Spiegel, Penev, \& Van Cauter, 2007; Knutson et al., 2009; Spiegel, Tasali, Penev, \& Van Cauter, 2004; Tasali, Leproult, Ehrmann, \& Van Cauter, 2008; Van Cauter et al., 2007).

\section{Conclusion and future directions}

The literature on caregivers clearly indicates that the intensity of chronic caregiving is associated with a multitude of health issues. From the psychological strains of providing care, to the physical toll and social stigma identified with caring for a mentally ill relative, the caregiving experience places a cumulative toll on the mind and body, contributing to increased AL and consequent morbidity and premature mortality. This is an example of complex dynamic interactions between behavior (caring for a relative), psychological issues (shame and guilt, general emotional distress), physiological outcomes (allostatic mechanisms), and social factors (social stigma or supporting environment) that collectively, through their interaction, exert powerful influence over important clinical outcomes.

The future direction of caregiver strain research could be to integrate multiple biomarkers to assess AL in caregivers as van Känel's group did looking at multiple dynamic biomarkers. This would reinforce the continued study of caregiving outcomes that tend to only focus on single biomarkers, if at all, and fall short of addressing effects on multiple physiological systems, or the downstream effects on physical or cognitive health debility. As higher AL is associated with poor health outcomes, undertaking various perspectives may help us better understand and implement clinical strategies to reduce and/or prevent the debilitating multifaceted effects of caregiving. 


\section{Transdisciplinary Allostatic Load Review}

\section{General Conclusion}

In order for the AL model to evolve from a research index into a clinical tool, it must become accessible for healthcare providers such as physicians, psychiatrists, and auxiliary professionals. Research has clearly substantiated that the AL index is sensitive to a wide array of stress-related conditions, and yet there is a perplexing reluctance to apply the AL model in practice and even more so as it relates to psychopathology. How could physiological algorithms possibly apply when testing and treating psychiatric patients? We hope that this review article is a rejoinder to this skepticism, as we believe strongly in the utility of an AL index in conjunction to interdisciplinary methodologies (e.g., combinations of psychosocial questionnaires, genetic assessment, routine physical check-ups, neuropsychological testing, clinical interviews, etc.) will facilitate person-centered approaches to research and practice alike. Furthermore, such biopsychosocial AL batteries could be used to comprehensively investigate the efficacies of interventions at multiple levels.

There are critical periods whereby the health sciences can identify individuals earlier on before AL leads to tertiary outcomes. This assertion stems from a population-based analysis of NHANES using more than twenty-two thousand participants, where it was shown that AL steadily increases with age up through the 20 s to 60 s and then plateaus throughout the 60 s to 90 s during the period of greatest mortality risk (Crimmins, Johnston, Hayward, \& Seeman, 2003). From life cycle and AL perspectives, this represents decade-long windows of opportunity to intervene before individuals succumb to disease and death; more than enough time to enact and instill positive health.

Because living with certain health conditions (e.g., severe mental illness, dementia, caregiving) can increase AL levels further, we believe the AL index could additionally be an effective tool to monitor disease courses and emergence of comorbidities. Even if most studies 


\section{Transdisciplinary Allostatic Load Review}

focus on physical outcomes, mortality rates, and the psychosocial antecedents of increased AL, the AL index shows tremendous promise in the study of psychopathological states as well. Just at a theoretical level, literature linking AL composites to psychopathologies like mood disorders (Kapczinski et al., 2008; McEwen, 2003c, 2004, 2005), anxiety disorders (McEwen, 2002b; Schulkin, Gold, \& McEwen, 1998; Schulkin, McEwen, \& Gold, 1994), and substance abuse (Koob, 2003; Koob et al., 2004; Koob \& Le Moal, 2001; Schulkin, 2003a; Valdez \& Koob, 2004) are very comprehensive, but unfortunately rarely applied to human studies.

A key theme emerging from the literature on psychopathology reviewed here is interaction. There are indeed complex interactions of hormones and biomarkers with neurophysiological systems and structures; constant interactions between biological systems; and profound interactions of biological, psychological, social, behavioral, and spiritual factors at the individual level. We have shown here that situations of early adversity, genetic and epigenetic elements, exposure to environmental toxins, sex and gender-related factors, and the experience of caregiving can in synergy exact profound influences on psychopathological outcomes. Guided by a developmental psychopathological approach (Cicchetti \& Rogosch, 2001; Cichetti \& Toth, 2009; Rutter \& Sroufe, 2000), we have also outlined the multidimensional implications of specific psychopathologies such as bipolar disorder, schizophrenia and the dementia spectrum as models of AL. A critical point to recognize is that these factors, which are directly or indirectly related to the individual, interact in complex ways to influence life-long disease susceptibilities. Understanding the nature and dynamics of these interactions, along with their impact on individual's psychological health require transdisciplinary approaches that include a broader analysis of the individual within society (Picard et al., 2011).

Beyond interaction, a transdisciplinary approach to $\mathrm{AL}$ also resembles transactional models previously outlined due to the bi-directional nature of the antecedents and the spectrum of 


\section{Transdisciplinary Allostatic Load Review}

consequences associated with chronic stress. Take for instance the central propositions of Bronfenbrenner's general ecological model whereby individual development is shaped by complex reciprocally interacting systems (Bronfenbrenner, 1977, 1994, 1995). These interdependent forces consist of the individual's immediate environment (micro-system), the interconnections among several individuals (meso-systems), the indirect influence of social structures and settings (exo-system), and finally the current overarching cultural and sub-cultural patterns (macro-system) over time (chrono-system). Consistent with the current review, we state that AL is clearly associated with SES, social/relationship, workplace, lifestyle, race/ethnic, and genetic factors throughout lifespan development. Targeting these antecedents of AL during key periods of development is therefore essential to improving public health. As such, our proposal of a trandisciplinary AL model shares similarities with transactional models already espoused in the field of developmental psychopathology (Sameroff, 2009; Sameroff, 2000; Sameroff \& Mackenzie, 2003).

Applying transdisciplinarity to psychopathology necessarily involves the creation of new knowledge emerging from the space between and beyond academic disciplines (see Figure 7). Disciplinary pillars must serve as solid foundations for an integrative process of inquiry directed at complex health problems. Research questions guided by real-life problems rather than determined by specialized disciplinary tools and perspectives and methodologies must be formulated. Establishing such transdisciplinary problems necessarily spanning multiple areas of research is the key consolidating ingredient to fruitful transdisciplinary research, rallying researchers towards sharing a common scientific objective (Kessel et al., 2008). Importantly, major funding agencies have recognized the need and the special challenges of multiple-investigator projects; as a result, several new interdisciplinary funding programs now exist (Kessel \& Rosenfield, 2008). For the field of psychopathology, adopting transdisciplinary research perspectives should allow 
researchers to integrate available evidence originating from multiple disciplines. Ultimately, one must position the individual and her/his complex transactional and multi-level experiences at the heart of scientific enquiry.

In conclusion, the AL model offers a useful framework for disciplines to come together with a common objective to impact individual's health. We believe that the AL model has potential to assist the progression towards transdisciplinary approaches to research, health care, and prevention for psychopathology throughout lifespan development.

\section{Acknowledgements}

We thank Catherine Juster for proof reading this manuscript. Further thanks go to Ilse Langerak for her review of stress and divorce. Funding for this article is thanks to a Doctoral scholarship awarded to Robert-Paul Juster from the Aging Institute of the Canadian Institutes of Health Research and to a Senior Investigator Chair from the Canadian Institute of Gender and Health to Sonia Lupien. 


\section{References}

Agirbasli, M., Agaoglu, N. B., Orak, N., Caglioz, H., Ocek, T., Karabag, T., \& Baykan, O. A. (2010). Sex hormones, insulin resistance and high-density lipoprotein cholesterol levels in children. Hormone Research in Paediatrics, 73(3), 166-174.

Agusa, T., Kunito, T., Iwata, H., Monirith, I., Chamnan, C., Tana, T. S., Subramanian, A., \& Tanabe, S. (2007). Mercury in hair and blood from residents of Phnom Penh (Cambodia) and possible effect on serum hormone levels. Chemosphere, 68(3), 590-596.

Albrecht, G., Freeman, S., \& Higginbotham, N. (1998). Complexity and human health: the case for a transdisciplinary paradigm. Culture, Medicine and Psychiatry, 22(1), 55-92.

Alley, D. E., Crimmins, E. M., Karlamangla, A., Hu, P., \& Seeman, T. E. (2008). Inflammation and rate of cognitive change in high-functioning older adults. The Journals of Gerontology. Series A, Biological Sciences and Medical Sciences, 63(1), 50-55.

Allison, D. B., Mentore, J. L., Heo, M., Chandler, L. P., Cappelleri, J. C., Infante, M. C., \& Weiden, P. J. (1999). Antipsychotic-induced weight gain: a comprehensive research synthesis. American Journal of Psychiatry, 156(11), 1686-1696.

Allsworth, J. E., Weitzen, S., \& Boardman, L. A. (2005). Early age at menarche and allostatic load: data from the Third National Health and Nutrition Examination Survey. Annals of Epidemiology, 15(6), 438-444. 
Amore, M. (2005). Partial androgen deficiency and neuropsychiatric symptoms in aging men. Journal of Endocrinological Investigation, 28(11 Suppl Proceedings), 49-54.

Ancelin, M. L., \& Ritchie, K. (2005). Lifelong endocrine fluctuations and related cognitive disorders. Current Pharmaceutical Design, 11(32), 4229-4252.

Arvanitakis, Z., Wilson, R. S., Bienias, J. L., Evans, D. A., \& Bennett, D. A. (2004). Diabetes mellitus and risk of Alzheimer disease and decline in cognitive function. [Research Support, U.S. Gov't, P.H.S.]. Archives of Neurology, 61(5), 661-666.

Asada, T., Kinoshita, T., \& Kakuma, T. (2000). Analysis of behavioral disturbances among community-dwelling elderly with Alzheimer disease. Alzheimer Disease and Associated Disorders, 14(3), 160-167.

Baccarelli, A., Mocarelli, P., Patterson, D. G., Jr., Bonzini, M., Pesatori, A. C., Caporaso, N., \& Landi, M. T. (2002). Immunologic effects of dioxin: new results from Seveso and comparison with other studies. Environmental Health Perspectives, 110(12), 1169-1173.

Baker, G. A., Santalo, R., \& Blumenstein, J. (1977). Effect of psychotropic agents upon the blastogenic response of human t-lymphocytes. Biological Psychiatry, 12(2), 159-169.

Baker, L. D., Frank, L. L., Foster-Schubert, K., Green, P. S., Wilkinson, C. W., McTiernan, A., Plymate, S. R., Fishel, M. A., Watson, G. S., Cholerton, B. A., Duncan, G. E., Mehta, P. D., \& Craft, S. (2010). Effects of aerobic exercise on mild cognitive impairment: a controlled trial. Archives of Neurology, 67(1), 71-79. 
Barr, A. M., Procyshyn, R. M., Hui, P., Johnson, J. L., \& Honer, W. G. (2008). Self-reported motivation to smoke in schizophrenia is related to antipsychotic drug treatment. Schizophrenia Research, 100(1-3), 252-260.

Barrett-Connor, E., \& Edelstein, S. L. (1994). A prospective study of dehydroepiandrosterone sulfate and cognitive function in an older population: the Rancho Bernardo Study. Journal of the American Geriatric Society, 42(4), 420-423.

Bauer, A. M., \& Boyce, W. T. (2004). Prophecies of childhood: how children's social environments and biological propensities affect the health of populations. International Journal of Behavioral Medicine, 11(3), 164-175.

Bearden, C. E., Thompson, P. M., Dalwani, M., Hayashi, K. M., Lee, A. D., Nicoletti, M., Trakhtenbroit, M., Glahn, D. C., Brambilla, P., Sassi, R. B., Mallinger, A. G., Frank, E., Kupfer, D. J., \& Soares, J. C. (2007). Greater cortical gray matter density in lithium-treated patients with bipolar disorder. Biological Psychiatry, 62(1), 7-16.

Behl, C. (2002). Oestrogen as a neuroprotective hormone. Nature Reviews. Neuroscience, 3(6), 433-442.

Bellingrath, S., Weigl, T., \& Kudielka, B. M. (2009). Chronic work stress and exhaustion is associated with higher allostastic load in female school teachers. Stress, 12(1), 37-48. 
Bennett, H. A., Einarson, A., Taddio, A., Koren, G., \& Einarson, T. R. (2004). Prevalence of depression during pregnancy: systematic review. Obstetrics and Gynecology International, 103(4), 698-709.

Berr, C., Lafont, S., Debuire, B., Dartigues, J. F., \& Baulieu, E. E. (1996). Relationships of dehydroepiandrosterone sulfate in the elderly with functional, psychological, and mental status, and short-term mortality: a French community-based study. Proceedings of the Natational Academy of Sciences of the United States of America, 93(23), 13410-13415.

Bilder, R. M., Goldman, R. S., Volavka, J., Czobor, P., Hoptman, M., Sheitman, B., Lindenmayer, J. P., Citrome, L., McEvoy, J., Kunz, M., Chakos, M., Cooper, T. B., Horowitz, T. L., \& Lieberman, J. A. (2002). Neurocognitive effects of clozapine, olanzapine, risperidone, and haloperidol in patients with chronic schizophrenia or schizoaffective disorder. American Journal of Psychiatry, 159(6), 1018-1028.

Bob, P., Raboch, J., Maes, M., Susta, M., Pavlat, J., Jasova, D., Vevera, J., Uhrova, J., Benakova, H., \& Zima, T. (2010). Depression, traumatic stress and interleukin-6. Journal of Affective Disorders, 120(1-3), 231-234.

Bonne, O., Brandes, D., Gilboa, A., Gomori, J. M., Shenton, M. E., Pitman, R. K., \& Shalev, A. Y. (2001). Longitudinal MRI study of hippocampal volume in trauma survivors with PTSD. American Journal of Psychiatry, 158(8), 1248-1251. 
Bookwala, J., \& Schulz, R. (2000). A comparison of primary stressors, secondary stressors, and depressive symptoms between elderly caregiving husbands and wives: the Caregiver Health Effects Study. Psychology and Aging, 15(4), 607-616.

Bouchard, M. F., Bellinger, D. C., Weuve, J., Matthews-Bellinger, J., Gilman, S. E., Wright, R. O., Schwartz, J., \& Weisskopf, M. G. (2009). Blood lead levels and major depressive disorder, panic disorder, and generalized anxiety disorder in US young adults. Archives of General Psychiatry, 66(12), 1313-1319.

Boucher, O., Muckle, G., \& Bastien, C. H. (2009). Prenatal Exposure to Polychlorinated Biphenyls: A Neuropsychologic Analysis. Environmental Health Perspectives, 117(1), 716.

Bouma, E. M., Riese, H., Ormel, J., Verhulst, F. C., \& Oldehinkel, A. J. (2009). Adolescents' cortisol responses to awakening and social stress; effects of gender, menstrual phase and oral contraceptives. The TRAILS study. Psychoneuroendocrinology, 34(6), 884-893.

Boyce, W. T., \& Ellis, B. J. (2005). Biological sensitivity to context: I. An evolutionarydevelopmental theory of the origins and functions of stress reactivity. Development and Psychopathology, 17(2), 271-301.

Bradley, R. H., \& Corwyn, R. F. (2002). Socioeconomic status and child development. Annual Review of Psychology, 53, 371-399. 
Brandes, M., Soares, C. N., \& Cohen, L. S. (2004). Postpartum onset obsessive-compulsive disorder: diagnosis and management. Archives of Women's Mental Health, 7(2), 99-110.

Braver, S. L., Ellman, I. M., \& Fabricius, W. V. (2003). Relocation of children after divorce and children's best interests: new evidence and legal considerations. Journal of Family Psychology, 17(2), 206-219.

Bremner, J. D., Randall, P., Vermetten, E., Staib, L., Bronen, R. A., Mazure, C., Capelli, S., McCarthy, G., Innis, R. B., \& Charney, D. S. (1997). Magnetic resonance imaging-based measurement of hippocampal volume in posttraumatic stress disorder related to childhood physical and sexual abuse--a preliminary report. Biological Psychiatry, 41(1), 23-32.

Bremner, J. D., Southwick, S. M., Johnson, D. R., Yehuda, R., \& Charney, D. S. (1993). Childhood physical abuse and combat-related posttraumatic stress disorder in Vietnam veterans. American Journal of Psychiatry, 150(2), 235-239.

Bremner, J. D., Vermetten, E., Afzal, N., \& Vythilingam, M. (2004). Deficits in verbal declarative memory function in women with childhood sexual abuse-related posttraumatic stress disorder. Journal of Nervous and Mental Disease, 192(10), 643-649.

Bremner, J. D., Vythilingam, M., Vermetten, E., Vaccarino, V., \& Charney, D. S. (2004). Deficits in hippocampal and anterior cingulate functioning during verbal declarative memory encoding in midlife major depression. American Journal of Psychiatry, 161(4), 637-645. 
Breunis, M. N., Kupka, R. W., Nolen, W. A., Suppes, T., Denicoff, K. D., Leverich, G. S., Post, R. M., \& Drexhage, H. A. (2003). High numbers of circulating activated T cells and raised levels of serum IL-2 receptor in bipolar disorder. Biological Psychiatry, 53(2), 157-165.

Brodeur, J. C., Sherwood, G., Rasmussen, J. B., \& Hontela, A. (1997). Impaired cortisol secretion in yellow perch (Perca flavescens) from lakes contaminated by heavy metals: in vivo and in vitro assessment. [Article]. Canadian Journal of Fisheries and Aquatic Sciences, 54(12), 2752-2758.

Brody, D. J., Pirkle, J. L., Kramer, R. A., Flegal, K. M., Matte, T. D., Gunter, E. W., \& Paschal, D. C. (1994). Blood Lead Levels in the Us Population - Phase-1 of the 3Rd NationalHealth and Nutrition Examination Survey (Nhanes-Iii, 1988 to 1991). Journal of the American Medical Association, 272(4), 277-283.

Bronfenbrenner, U. (1977). Toward an experimental ecology of human development. American Psychologist, 32, 513-531.

Bronfenbrenner, U. (1994). Ecological models of human development Interactional Encyclopedia of Education (2 ed., Vol. 3). Oxford: Elsevier.

Bronfenbrenner, U. (1995). Developmental ecology through space and time: a future perspective. In P. Moen, G. H. Elder \& K. Luscher (Eds.), Examining lives in context: perspectives on the ecology of human development (pp. 619-647). Washington, D.C.: American Psychological Association. 
Brosschot, J. F., Gerin, W., \& Thayer, J. F. (2006). The perseverative cognition hypothesis: a review of worry, prolonged stress-related physiological activation, and health. Journal of Psychosomatic Research, 60(2), 113-124.

Brosschot, J. F., Pieper, S., \& Thayer, J. F. (2005). Expanding stress theory: prolonged activation and perseverative cognition. Psychoneuroendocrinology, 30(10), 1043-1049.

Brosschot, J. F., \& Thayer, J. F. (2003). Heart rate response is longer after negative emotions than after positive emotions. International Journal of Psychophysiology, 50(3), 181-187.

Bruehl, H., Rueger, M., Dziobek, I., Sweat, V., Tirsi, A., Javier, E., Arentoft, A., Wolf, O. T., \& Convit, A. (2007). Hypothalamic-pituitary-adrenal axis dysregulation and memory impairments in type 2 diabetes. Journal of Clinical Endocrinology \& Metabolism, 92(7), 2439-2445.

Brundtland, G. H. (2001). From the World Health Organization. Mental health: new understanding, new hope. Journal of the American Medical Association, 286(19), 2391.

Bruns, C. M., \& Kemnitz, J. W. (2004). Sex hormones, insulin sensitivity, and diabetes mellitus. ILAR Journal/National Research Council, Institute of Laboratory Animal Resources, 45(2), 160-169.

Bschor, T., Adli, M., Baethge, C., Eichmann, U., Ising, M., Uhr, M., Modell, S., Kunzel, H., Muller-Oerlinghausen, B., \& Bauer, M. (2002). Lithium augmentation increases the ACTH 


\section{Transdisciplinary Allostatic Load Review}

and cortisol response in the combined $\mathrm{DEX} / \mathrm{CRH}$ test in unipolar major depression. Neuropsychopharmacology, 27(3), 470-478.

Buchanan, T. W., \& Lovallo, W. R. (2001). Enhanced memory for emotional material following stress-level cortisol treatment in humans. Psychoneuroendocrinology, 26(3), 307-317.

Burgmans, S., van Boxtel, M. P., Vuurman, E. F., Smeets, F., Gronenschild, E. H., Uylings, H. B., \& Jolles, J. (2009). The prevalence of cortical gray matter atrophy may be overestimated in the healthy aging brain. Neuropsychology, 23(5), 541-550.

Burke, H. M., Davis, M. C., Otte, C., \& Mohr, D. C. (2005). Depression and cortisol responses to psychological stress: a meta-analysis. Psychoneuroendocrinology, 30(9), 846-856.

Burns, J. M., Baghurst, P. A., Sawyer, M. G., McMichael, A. J., \& Tong, S. L. (1999). Lifetime low-level exposure to environmental lead and children's emotional and behavioral development at ages 11-13 years. The Port Pirie Cohort Study. American Journal of Epidemiology, 149(8), 740-749.

Burt, D. B., Zembar, M. J., \& Niederehe, G. (1995). Depression and memory impairment: a metaanalysis of the association, its pattern, and specificity. Psychological Bulletin, 117(2), 285305.

Burton, L. C., Newsom, J. T., Schulz, R., Hirsch, C. H., \& German, P. S. (1997). Preventive health behaviors among spousal caregivers. Preventative Medicine, 26(2), 162-169. 


\section{Transdisciplinary Allostatic Load Review}

Buss, C., Lord, C., Wadiwalla, M., Hellhammer, D. H., Lupien, S. J., Meaney, M. J., \& Pruessner, J. C. (2007). Maternal care modulates the relationship between prenatal risk and hippocampal volume in women but not in men. Journal of Neuroscience, 27(10), 25922595.

Cacioppo, J. T., Poehlmann, K. M., Kiecolt-Glaser, J. K., Malarkey, W. B., Burleson, M. H., Berntson, G. G., \& Glaser, R. (1998). Cellular immune responses to acute stress in female caregivers of dementia patients and matched controls. Health Psychology, 17(2), 182-189.

Campbell, S., Marriott, M., Nahmias, C., \& MacQueen, G. M. (2004). Lower hippocampal volume in patients suffering from depression: A meta-analysis. American Journal of Psychiatry, 161(4), 598-607.

Canfield, R. L., Henderson, C. R., Jr., Cory-Slechta, D. A., Cox, C., Jusko, T. A., \& Lanphear, B. P. (2003). Intellectual impairment in children with blood lead concentrations below 10 microg per deciliter. New England Journal of Medicine, 348(16), 1517-1526.

Canli, T., Qiu, M., Omura, K., Congdon, E., Haas, B. W., Amin, Z., Herrmann, M. J., Constable, R. T., \& Lesch, K. P. (2006). Neural correlates of epigenesis. Proceedings of the National Academy of Science of the United States of America, 103(43), 16033-16038.

Carrion, V. G., Weems, C. F., Eliez, S., Patwardhan, A., Brown, W., Ray, R. D., \& Reiss, A. L. (2001). Attenuation of frontal asymmetry in pediatric posttraumatic stress disorder. Biological Psychiatry, 50(12), 943-951. 
Carter, P. A., \& Chang, B. L. (2000). Sleep and depression in cancer caregivers. Cancer Nursing, $23(6), 410-415$.

Caspi, A., Sugden, K., Moffitt, T. E., Taylor, A., Craig, I. W., Harrington, H., McClay, J., Mill, J., Martin, J., Braithwaite, A., \& Poulton, R. (2003). Influence of life stress on depression: moderation by a polymorphism in the 5-HTT gene. Science, 301(5631), 386-389.

Centers for Disease, C., \& Prevention. (2005). Third National Report on Human Exposure to Environmental Chemicals. Atlanta (GA).

Champagne, F., \& Meaney, M. J. (2001). Like mother, like daughter: evidence for non-genomic transmission of parental behavior and stress responsivity. Progress in Brain Research, 133, 287-302.

Chan, H. M., \& Egeland, G. M. (2004). Fish consumption, mercury exposure, and heart diseases. Nutrition Reviews, 62(2), 68-72.

Chase-Lansdale, P. L., Cherlin, A. J., \& Kiernan, K. E. (1995). The long-term effects of parental divorce on the mental health of young adults: a developmental perspective. Child Development, 66(6), 1614-1634.

Chen, J., Lipska, B. K., Halim, N., Ma, Q. D., Matsumoto, M., Melhem, S., Kolachana, B. S., Hyde, T. M., Herman, M. M., Apud, J., Egan, M. F., Kleinman, J. E., \& Weinberger, D. R. (2004). Functional analysis of genetic variation in catechol-O-methyltransferase (COMT): 
effects on mRNA, protein, and enzyme activity in postmortem human brain. American Journal of Human Genetics, 75(5), 807-821.

Christensen, M. V., Kyvik, K. O., \& Kessing, L. V. (2006). Cognitive function in unaffected twins discordant for affective disorder. Psychological Medicine, 36(8), 1119-1129.

Chuang, H. Y., Yu, K. T., Ho, C. K., Wu, M. T., Lin, G. T., \& Wu, T. N. (2004). Investigations of vitamin D receptor polymorphism affecting workers' susceptibility to lead. Journal of Occupational Health, 46(4), 316-322.

Cicchetti, D. (2002). The impact of social experience on neurobiological systems: Illustration from a constructivist view of child maltreatment. Cognitive Development, 17(3), 14071428.

Cicchetti, D., \& Rogosch, F. A. (2001). Diverse patterns of neuroendocrine activity in maltreated children. Development and Psychopathology, 13(3), 677-693.

Cicchetti, D., Rogosch, F. A., Gunnar, M. R., \& Toth, S. L. (2010a). The differential impacts of early physical and sexual abuse and internalizing problems on daytime cortisol rhythm in school-aged children. Child Development, 81(1), 252-269.

Cicchetti, D., Rogosch, F. A., Howe, M. L., \& Toth, S. L. (2010b). The effects of maltreatment and neuroendocrine regulation on memory performance. Child Development, 81(5), 15041519. 
Cichetti, D., \& Toth, S. L. (2009). The past achievements and future promises of developmental psychopathology: the coming of age of a discipline. Journal of Child Psychology and Psychiatry, 50(1-2), 16-25.

Clark, M. S., Bond, M. J., \& Hecker, J. R. (2007). Environmental stress, psychological stress and allostatic load. Psychology, Health and Medicine, 12(1), 18-30.

Cohrs, S., Roher, C., Jordan, W., Meier, A., Huether, G., Wuttke, W., Ruther, E., \& Rodenbeck, A. (2006). The atypical antipsychotics olanzapine and quetiapine, but not haloperidol, reduce ACTH and cortisol secretion in healthy subjects. Psychopharmacology (Berlin), 185(1), 11-18.

Colcombe, S. J., Kramer, A. F., McAuley, E., Erickson, K. I., \& Scalf, P. (2004). Neurocognitive aging and cardiovascular fitness: recent findings and future directions. Journal of Molecular Neuroscience, 24(1), 9-14.

Convit, A. (2005). Links between cognitive impairment in insulin resistance: an explanatory model. Neurobiology of Aging, 26 Suppl 1, 31-35.

Convit, A., Wolf, O. T., Tarshish, C., \& de Leon, M. J. (2003). Reduced glucose tolerance is associated with poor memory performance and hippocampal atrophy among normal elderly. Proceedings of the National Academy of Sciences of the United States of America, 100(4), 2019-2022. 
Copinschi, G., Nedeltcheva, A., Leproult, R., Morselli, L. L., Spiegel, K., Martino, E., Legros, J. J., Weiss, R. E., Mockel, J., \& Van Cauter, E. (2010). Sleep disturbances, daytime sleepiness, and quality of life in adults with growth hormone deficiency. Journal of Clinical Endocrinology and Metabolism, 95(5), 2195-2202.

Cornblatt, B., Carson, W. H., Ali, M., Kern, R. S., Luo, X., \& Green, M. (2002). Neurocognitive effects of aripiprazole vs olanzapine [abstr.]. International Journal of Neuropsychopharmacology, 5(Suppl 1), S158.

Cornblatt, B. A., Lencz, T., Smith, C. W., Olsen, R., Auther, A. M., Nakayama, E., Lesser, M. L., Tai, J. Y., Shah, M. R., Foley, C. A., Kane, J. M., \& Correll, C. U. (2007). Can antidepressants be used to treat the schizophrenia prodrome? Results of a prospective, naturalistic treatment study of adolescents. Journal of Clinical Psychiatry, 68(4), 546-557.

Cory-Slechta, D. A., Virgolini, M. B., Rossi-George, A., Thiruchelvam, M., Lisek, R., \& Weston, D. (2008). Lifetime consequences of combined maternal lead and stress. Basic and Clinical Pharmacology and Toxicology, 102(2), 218-227.

Cory-Slechta, D. A., Virgolini, M. B., Thiruchelvam, M., Weston, D. D., \& Bauter, M. R. (2004). Maternal stress modulates the effects of developmental lead exposure. Environmental Health Perspectives, 112(6), 717-730.

Courtenay, W. H. (2000). Constructions of masculinity and their influence on men's well-being: a theory of gender and health. Social Science and Medicine, 50(10), 1385-1401. 
Craddock, N., Owen, M. J., \& O'Donovan, M. C. (2006). The catechol-O-methyl transferase (COMT) gene as a candidate for psychiatric phenotypes: evidence and lessons. Molecular Psychiatry, 11(5), 446-458.

Creese, J., Bedard, M., Brazil, K., \& Chambers, L. (2008). Sleep disturbances in spousal caregivers of individuals with Alzheimer's disease. International Psychogeriatrics, 20(1), 149-161.

Crews, D. E. (2007). Composite estimates of physiological stress, age, and diabetes in American Samoans. American Journal of Physical Anthropology, 133(3), 1028-1034.

Crimmins, E. M., Johnston, M., Hayward, M., \& Seeman, T. (2003). Age differences in allostatic load: an index of physiological dysregulation. Experimental Gerontology, 38(7), 731-734.

Csernansky, J. G., Dong, H., Fagan, A. M., Wang, L., Xiong, C., Holtzman, D. M., \& Morris, J. C. (2006). Plasma cortisol and progression of dementia in subjects with Alzheimer-type dementia. American Journal of Psychiatry, 163(12), 2164-2169.

Cutolo, M., Capellino, S., \& Straub, R. H. (2008). Oestrogens in rheumatic diseases: friend or foe? Rheumatology (Oxford), 47 Suppl 3, 2-5.

Cutolo, M., \& Straub, R. H. (2009). Insights into endocrine-immunological disturbances in autoimmunity and their impact on treatment. Arthritis Research and Therapy, 11(2), 218. 
Cutolo, M., Sulli, A., Capellino, S., Villaggio, B., Montagna, P., Seriolo, B., \& Straub, R. H. (2004). Sex hormones influence on the immune system: basic and clinical aspects in autoimmunity. Lupus, 13(9), 635-638.

Czyrak, A., Mackowiak, M., Chocyk, A., Fijal, K., \& Wedzony, K. (2003). Role of glucocorticoids in the regulation of dopaminergic neurotransmission. Polish Journal of Pharmacology, 55(5), 667-674.

Dakhale, G., Khanzode, S., Saoji, A., Khobragade, L., \& Turankar, A. (2004). Oxidative damage and schizophrenia: the potential benefit by atypical antipsychotics. Neuropsychobiology, 49(4), 205-209.

Dallaire, F., Dewailly, E., Vezina, C., Muckle, G., Weber, J. P., Bruneau, S., \& Ayotte, P. (2006). Effect of prenatal exposure to polychlorinated biphenyls on incidence of acute respiratory infections in preschool Inuit children. Environmental Health Perspectives, 114(8), 13011305.

Dallman, M. F., Akana, S. F., Strack, A. M., Scribner, K. S., Pecoraro, N., La Fleur, S. E., Houshyar, H., \& Gomez, F. (2004). Chronic stress-induced effects of corticosterone on brain: direct and indirect. Annals of the New York Academy of Sciences, 1018, 141-150.

Darbre, P. D. (2006). Metalloestrogens: an emerging class of inorganic xenoestrogens with potential to add to the oestrogenic burden of the human breast. Journal of Applied Toxicology, 26(3), 191-197. 


\section{Transdisciplinary Allostatic Load Review}

Davidson, P. W., Myers, G. J., Cox, C., Axtell, C., Shamlaye, C., Sloane-Reeves, J., Cernichiari, E., Needham, L., Choi, A., Wang, Y., Berlin, M., \& Clarkson, T. W. (1998). Effects of prenatal and postnatal methylmercury exposure from fish consumption on neurodevelopment: outcomes at 66 months of age in the Seychelles Child Development Study. Journal of the American Medical Association, 280(8), 701-707.

Davis, L. L., Weaver, M., Zamrini, E., Stevens, A., Kang, D. H., \& Parker, C. R., Jr. (2004). Biopsychological markers of distress in informal caregivers. Biological Research for Nursing, 6(2), 90-99.

De Bellis, M. D., Hall, J., Boring, A. M., Frustaci, K., \& Moritz, G. (2001). A pilot longitudinal study of hippocampal volumes in pediatric maltreatment-related posttraumatic stress disorder. Biological Psychiatry, 50(4), 305-309.

De Bellis, M. D., Keshavan, M. S., Clark, D. B., Casey, B. J., Giedd, J. N., Boring, A. M., Frustaci, K., \& Ryan, N. D. (1999). A.E. Bennett Research Award. Developmental traumatology. Part II: Brain development. Biological Psychiatry, 45(10), 1271-1284.

De Geus, E. J., Kupper, N., Boomsma, D. I., \& Snieder, H. (2007). Bivariate genetic modeling of cardiovascular stress reactivity: does stress uncover genetic variance? Psychosomatic Medicine, 69(4), 356-364.

de Mamani, A. G. (2010). Self-conscious emotions, general emotional distress, and expressed emotion in family members of patients with schizophrenia. Journal of Nervous and Mental Disease, 198(4), 305-308. 


\section{Transdisciplinary Allostatic Load Review}

de Vugt, M. E., Nicolson, N. A., Aalten, P., Lousberg, R., Jolle, J., \& Verhey, F. R. (2005). Behavioral problems in dementia patients and salivary cortisol patterns in caregivers. Journal of Neuropsychiatry and Clinical Neuroscience, 17(2), 201-207.

Debes, F., Budtz-Jorgensen, E., Weihe, P., White, R. F., \& Grandjean, P. (2006). Impact of prenatal methylmercury exposure on neurobehavioral function at age 14 years. Neurotoxicology and Teratology, 28(3), 363-375.

Dedovic, K., Duchesne, A., Andrews, J., Engert, V., \& Pruessner, J. C. (2009). The brain and the stress axis: The neural correlates of cortisol regulation in response to stress. Neuroimage, 47(3), 864-871.

Denham, M., Schell, L. M., Deane, G., Gallo, M. V., Ravenscroft, J., \& DeCaprio, A. P. (2005). Relationship of lead, mercury, mirex, dichlorodiphenyldichloroethylene, hexachlorobenzene, and polychlorinated biphenyls to timing of menarche among Akwesasne Mohawk girls. Pediatrics, 115(2), E127-E134.

Denicoff, K. D., Rubinow, D. R., Papa, M. Z., Simpson, C., Seipp, C. A., Lotze, M. T., Chang, A. E., Rosenstein, D., \& Rosenberg, S. A. (1987). The neuropsychiatric effects of treatment with interleukin-2 and lymphokine-activated killer cells. Annals of Internal Medicine, 107(3), 293-300.

Dickerson, B. C., Goncharova, I., Sullivan, M. P., Forchetti, C., Wilson, R. S., Bennett, D. A., Beckett, L. A., \& deToledo-Morrell, L. (2001). MRI-derived entorhinal and hippocampal 


\section{Transdisciplinary Allostatic Load Review}

atrophy in incipient and very mild Alzheimer's disease. [Research Support, U.S. Gov't, P.H.S.]. Neurobiology of Aging, 22(5), 747-754.

Dickerson, S. S., \& Kemeny, M. E. (2004). Acute stressors and cortisol responses: a theoretical integration and synthesis of laboratory research. Psychological Bulliten, 130(3), 355-391.

Dik, M. G., Jonker, C., Hack, C. E., Smit, J. H., Comijs, H. C., \& Eikelenboom, P. (2005). Serum inflammatory proteins and cognitive decline in older persons. Neurology, 64(8), 13711377.

Dobbs, D. (2009). Orchid Children. The Atlantic, 60-68.

Dowd, J. B., Simanek, A. M., \& Aiello, A. E. (2009). Socio-economic status, cortisol and allostatic load: a review of the literature. International Journal of Epidemiology, 38(5), 1297-1309.

Du, A. T., Schuff, N., Chao, L. L., Kornak, J., Jagust, W. J., Kramer, J. H., Reed, B. R., Miller, B. L., Norman, D., Chui, H. C., \& Weiner, M. W. (2006). Age effects on atrophy rates of entorhinal cortex and hippocampus. Neurobiology of Aging, 27(5), 733-740.

Duman, R. S., Nakagawa, S., \& Malberg, J. (2001). Regulation of adult neurogenesis by antidepressant treatment. Neuropsychopharmacology, 25(6), 836-844.

Eberling, J. L., Reed, B. R., Coleman, J. E., \& Jagust, W. J. (2000). Effect of estrogen on cerebral glucose metabolism in postmenopausal women. Neurology, 55(6), 875-877. 
Eberling, J. L., Wu, C., Haan, M. N., Mungas, D., Buonocore, M., \& Jagust, W. J. (2003). Preliminary evidence that estrogen protects against age-related hippocampal atrophy. Neurobiology of Aging, 24(5), 725-732.

Elder, S. J., Lichtenstein, A. H., Pittas, A. G., Roberts, S. B., Fuss, P. J., Greenberg, A. S., McCrory, M. A., Bouchard, T. J., Jr., Saltzman, E., \& Neale, M. C. (2009). Genetic and environmental influences on factors associated with cardiovascular disease and the metabolic syndrome. Journal of Lipid Research, 50(9), 1917-1926.

Ellingrod, V. L., Perry, P. J., Ringold, J. C., Lund, B. C., Bever-Stille, K., Fleming, F., Holman, T. L., \& Miller, D. (2005). Weight gain associated with the $-759 \mathrm{C} / \mathrm{T}$ polymorphism of the 5HT2C receptor and olanzapine. American Journal of Medical Genetics. Part B, Neuropsychiatric Genetics, 134B(1), 76-78.

Elman, I., Borsook, D., \& Lukas, S. E. (2006). Food intake and reward mechanisms in patients with schizophrenia: implications for metabolic disturbances and treatment with secondgeneration antipsychotic agents. Neuropsychopharmacology, 31(10), 2091-2120.

Engelhart, M. J., Geerlings, M. I., Meijer, J., Kiliaan, A., Ruitenberg, A., van Swieten, J. C., Stijnen, T., Hofman, A., Witteman, J. C., \& Breteler, M. M. (2004). Inflammatory proteins in plasma and the risk of dementia: the rotterdam study. Archives of Neurology, 61(5), 668-672.

Epel, E. S. (2009). Psychological and metabolic stress: a recipe for accelerated cellular aging? Hormones (Athens), 8(1), 7-22. 
Epel, E. S., Blackburn, E. H., Lin, J., Dhabhar, F. S., Adler, N. E., Morrow, J. D., \& Cawthon, R. M. (2004). Accelerated telomere shortening in response to life stress. Proceeds of the National Acedemy of Sciences of the United States of America, 101(49), 17312-17315.

Erickson, K. I., Prakash, R. S., Voss, M. W., Chaddock, L., Hu, L., Morris, K. S., White, S. M., Wojcicki, T. R., McAuley, E., \& Kramer, A. F. (2009). Aerobic fitness is associated with hippocampal volume in elderly humans. Hippocampus, 19(10), 1030-1039.

Esser, C., \& Welzel, M. (1993). Ontogenic development of murine fetal thymocytes is accelerated by 3,3',4,4'-tetrachlorobiphenyl. Internation Journal of Immunopharmacology, 15(8), 841852.

Esterling, B. A., Kiecolt-Glaser, J. K., \& Glaser, R. (1996). Psychosocial modulation of cytokineinduced natural killer cell activity in older adults. Psychosomstic Medicine, 58(3), 264272.

Etgen, T., Sander, D., Huntgeburth, U., Poppert, H., Forstl, H., \& Bickel, H. (2010). Physical activity and incident cognitive impairment in elderly persons: the INVADE study. Archives of Internal Medicine, 170(2), 186-193.

Evans, G. W. (2003). A multimethodological analysis of cumulative risk and allostatic load among rural children. Developmental Psychology, 39(5), 924-933. 
Evans, G. W., Kim, P., Ting, A. H., Tesher, H. B., \& Shannis, D. (2007). Cumulative risk, maternal responsiveness, and allostatic load among young adolescents. Developmental Psychology, 43(2), 341-351.

Evans, G. W., \& Schamberg, M. A. (2009). Childhood poverty, chronic stress, and adult working memory. Proceeds of the National Acedemy of Sciences of the United States of America, $106(16), 6545-6549$.

Everett, C. J., King, D. E., Player, M. S., Matheson, E. M., Post, R. E., \& Mainous, A. G., 3rd. (2010). Association of urinary polycyclic aromatic hydrocarbons and serum C-reactive protein. Environmental Research, 110(1), 79-82.

Feldman, H. A., Longcope, C., Derby, C. A., Johannes, C. B., Araujo, A. B., Coviello, A. D., Bremner, W. J., \& McKinlay, J. B. (2002). Age trends in the level of serum testosterone and other hormones in middle-aged men: longitudinal results from the Massachusetts male aging study. Journal of Clinical and Endocrinology and Metabolism, 87(2), 589-598.

Fewtrell, L. J., Pruss-Ustun, A., Landrigan, P., \& yuso-Mateos, J. L. (2004). Estimating the global burden of disease of mild mental retardation and cardiovascular diseases from environmental lead exposure. Environmental Research, 94(2), 120-133.

Fillion, M., Mergler, D., Sousa Passos, C. J., Larribe, F., Lemire, M., \& Guimaraes, J. R. (2006). A preliminary study of mercury exposure and blood pressure in the Brazilian Amazon. Environmental Health, 5, 29. 
Fiocco, A. J., Poirier, J., Joober, R., Nair, N. P., \& Lupien, S. J. (2008). Acute and long-term associations between ApoE genetic polymorphism, cortisol levels, and declarative memory performance in older adults. Psychoneuroendocrinology, 33(5), 625-633.

Fischer, J. C., Kudielka, B. M., von Kanel, R., Siegrist, J., Thayer, J. F., \& Fischer, J. E. (2009). Bone-marrow derived progenitor cells are associated with psychosocial determinants of health after controlling for classical biological and behavioral cardiovascular risk factors. Brain, Behavior, and Immunity, 23(4), 419-426.

Flaskerud, J. H., Carter, P. A., \& Lee, P. (2000). Distressing emotions in female caregivers of people with AIDS, age-related dementias, and advanced-stage cancers. Perspectives in Psychiatric Care, 36(4), 121-130.

Foland, L. C., Altshuler, L. L., Sugar, C. A., Lee, A. D., Leow, A. D., Townsend, J., Narr, K. L., Asuncion, D. M., Toga, A. W., \& Thompson, P. M. (2008). Increased volume of the amygdala and hippocampus in bipolar patients treated with lithium. Neuroreport, 19(2), 221-224.

Forti, P., Pisacane, N., Rietti, E., Lucicesare, A., Olivelli, V., Mariani, E., Mecocci, P., \& Ravaglia, G. (2010). Metabolic Syndrome and Risk of Dementia in Older Adults. Journal of American Geriatrics Society, 58, 487-492.

Fossati, P., Harvey, P. O., Le Bastard, G., Ergis, A. M., Jouvent, R., \& Allilaire, J. F. (2004). Verbal memory performance of patients with a first depressive episode and patients with 
unipolar and bipolar recurrent depression. Journal of Psychiatric Research, 38(2), 137144.

Francis, D., Diorio, J., Liu, D., \& Meaney, M. J. (1999). Nongenomic transmission across generations of maternal behavior and stress responses in the rat. Science, 286(5442), 11551158.

Fried, L. P., Tangen, C. M., Walston, J., Newman, A. B., Hirsch, C., Gottdiener, J., Seeman, T., Tracy, R., Kop, W. J., Burke, G., \& McBurnie, M. A. (2001). Frailty in older adults: evidence for a phenotype. The Journals of Gerontology. Series A, Biological Sciences and Medical Sciences, 56(3), M146-156.

Fries, J. H., Hellhammer, J., \& Hellhammer, D. H. (2005). A new view of hypocortisolism. Psychoneuroendocrinology, 30(10), 1010-1016.

Furumoto-Dawson, A., Gehlert, S., Sohmer, D., Olopade, O., \& Sacks, T. (2007). Early-life conditions and mechanisms of population health vulnerabilities. Health Affairs (Millwood), $26(5), 1238-1248$.

Gallacher, J., Bayer, A., Lowe, G., Fish, M., Pickering, J., Pedro, S., Dunstan, F., White, J., Yarnell, J., \& Ben-Shlomo, Y. (2010). Is sticky blood bad for the brain?: Hemostatic and inflammatory systems and dementia in the Caerphilly Prospective Study. Arteriosclerosis, Thrombosis, and Vascular Biology, 30(3), 599-604. 
Gallagher, P., Watson, S., Smith, M. S., Young, A. H., \& Ferrier, I. N. (2007). Plasma cortisoldehydroepiandrosterone (DHEA) ratios in schizophrenia and bipolar disorder. Schizophrenia Research, 90(1-3), 258-265.

Ganzel, B. L., Morris, P. A., \& Wethington, E. (2010). Allostasis and the human brain: Integrating models of stress from the social and life sciences. Psychological Review, 117(1), 134-174.

Garg, R., \& Yusuf, S. (1995). Overview of randomized trials of angiotensin-converting enzyme inhibitors on mortality and morbidity in patients with heart failure. Collaborative Group on ACE Inhibitor Trials. Journal of the American Medical Association, 273(18), 1450-1456.

Geerlings, M. I., den Heijer, T., Koudstaal, P. J., Hofman, A., \& Breteler, M. M. (2008). History of depression, depressive symptoms, and medial temporal lobe atrophy and the risk of Alzheimer disease. Neurology, 70(15), 1258-1264.

Geerlings, M. I., Ruitenberg, A., Witteman, J. C., van Swieten, J. C., Hofman, A., van Duijn, C. M., Breteler, M. M., \& Launer, L. J. (2001). Reproductive period and risk of dementia in postmenopausal women. Journal of the American Medical Association, 285(11), 14751481.

Gendron, A. D., Bishop, C. A., Fortin, R., \& Hontela, A. (1997). In vivo testing of the functional integrity of the corticosterone-producing axis in mudpuppy (amphibia) exposed to chlorinated hydrocarbons in the wild. Environmental Toxicology and Chemistry, 16(8), 1694-1706. 
Geronimus, A. T., Hicken, M., Keene, D., \& Bound, J. (2006). "Weathering" and age patterns of allostatic load scores among blacks and whites in the United States. American Journal of Public Health, 96(5), 826-833.

Gerritsen, L., Comijs, H. C., Deeg, D. J., Penninx, B. W., \& Geerlings, M. I. (2009). Salivary cortisol, APOE-varepsilon4 allele and cognitive decline in a prospective study of older persons. Neurobiology of Aging.

Gianaros, P. J., Jennings, J. R., Sheu, L. K., Greer, P. J., Kuller, L. H., \& Matthews, K. A. (2007). Prospective reports of chronic life stress predict decreased grey matter volume in the hippocampus. Neuroimage, 35(2), 795-803.

Giedd, J. N., Snell, J. W., Lange, N., Rajapakse, J. C., Casey, B. J., Kozuch, P. L., Vaituzis, A. C., Vauss, Y. C., Hamburger, S. D., Kaysen, D., \& Rapoport, J. L. (1996). Quantitative magnetic resonance imaging of human brain development: ages 4-18. Cerebral Cortex, 6(4), 551-560.

Gilbert, S. G., \& Grantwebster, K. S. (1995). Neurobehavioral Effects of Developmental Methylmercury Exposure. Environmental Health Perspectives, 103, 135-142.

Gilbert, S. G., \& Weiss, B. (2006). A rationale for lowering the blood lead action level from 10 to 2 microg/dL. Neurotoxicology, 27(5), 693-701. 
Gilbertson, M. W., Shenton, M. E., Ciszewski, A., Kasai, K., Lasko, N. B., Orr, S. P., \& Pitman, R. K. (2002). Smaller hippocampal volume predicts pathologic vulnerability to psychological trauma. Nature Neuroscience, 5(11), 1242-1247.

Giubilei, F., Patacchioli, F. R., Antonini, G., Sepe Monti, M., Tisei, P., Bastianello, S., Monnazzi, P., \& Angelucci, L. (2001). Altered circadian cortisol secretion in Alzheimer's disease: clinical and neuroradiological aspects. Journal of Neuroscience Research, 66(2), 262-265.

Glei, D. A., Goldman, N., Chuang, Y. L., \& Weinstein, M. (2007). Do chronic stressors lead to physiological dysregulation? Testing the theory of allostatic load. Psychosomatic Medicine, 69(8), 769-776.

Glover, D. A. (2006). Allostatic load in women with and without PTSD symptoms. Annals of the New York Academy of Science, 442-447.

Glover, D. A., Garcia-Arcena, E. F., \& Mohlman, J. (2008). Peripheral biomarker composite associated with smaller hippocampal volume. NeuroReport, 19(13), 1313-1316.

Glover, D. A., Stuber, M., \& Poland, R. E. (2006). Allostatic load in women with and without PTSD symptoms. Psychiatry, 69(3), 191-203.

Godfrey, M. E., Wojcik, D. P., \& Krone, C. A. (2003). Apolipoprotein E genotyping as a potential biomarker for mercury neurotoxicity. Journal of Alzheimers Disease, 5(3), 189-195. 
Goertzel, B. N., Pennachin, C., de Souza Coelho, L., Maloney, E. M., Jones, J. F., \& Gurbaxani, B. (2006). Allostatic load is associated with symptoms in chronic fatigue syndrome patients. Pharmacogenomics, 7(3), 485-494.

Gold, S. M., Dziobek, I., Sweat, V., Tirsi, A., Rogers, K., Bruehl, H., Tsui, W., Richardson, S., Javier, E., \& Convit, A. (2007). Hippocampal damage and memory impairments as possible early brain complications of type 2 diabetes. Diabetologia, 50(4), 711-719.

Goldman, N., Glei, D., Seplaki, C. L., Lui, I. W., \& Weinstein, M. (2005). Perceived stress and physiological dysregulations in older adults. Stress, 8(2), 95-105.

Goldman, N., Turra, C. M., Glei, D. A., Lin, Y. H., \& Weinstein, M. (2006a). Physiological dysregulation and changes in health in an older population. Experimental Gerontology, $41(9), 862-870$.

Goldman, N., Turra, C. M., Glei, D. A., Seplaki, C. L., Lin, Y. H., \& Weinstein, M. (2006b). Predicting mortality from clinical and nonclinical biomarkers. Journals of Gerontology. Series A: Biological Sciences and Medical Sciences, 61(10), 1070-1074.

Goldstein, D. S., \& Kopin, I. J. (2007). Evolution of concepts of stress. Stress, 10(2), 109-120.

Gomby, D. S., Larner, M. B., Stevenson, C. S., Lewit, E. M., \& Behrman, R. E. (1995). Longterm outcomes of early childhood programs: analysis and recommendations. Future Child, 5(3), 6-24. 
Goncharov, A., Haase, R. F., Santiago-Rivera, A., Morse, G., McCaffrey, R. J., Rej, R., \& Carpenter, D. O. (2008). High serum PCBs are associated with elevation of serum lipids and cardiovascular disease in a Native American population. Environmental Research, $106(2), 226-239$.

Goodman, E., McEwen, B. S., Huang, B., Dolan, L. M., \& Adler, N. E. (2005). Social inequalities in biomarkers of cardiovascular risk in adolescence. Psychosomatic Medicine, 67(1), 9-15.

Gore, S., Aseltine, R. H., Jr., \& Colton, M. E. (1992). Social structure, life stress and depressive symptoms in a high school-aged population. Journal of Health and Social Behavior, 33(2), 97-113.

Gotlib, I. H., Joormann, J., Minor, K. L., \& Hallmayer, J. (2008). HPA axis reactivity: a mechanism underlying the associations among 5-HTTLPR, stress, and depression. Biological Psychiatry, 63(9), 847-851.

Goymann, W., \& Wingfield, J. C. (2004). Allostatic load, social status and stress hormones: the costs of social status matter. Animal Behaviour, 67(3), 591-602.

Grady, D., Herrington, D., Bittner, V., Blumenthal, R., Davidson, M., Hlatky, M., Hsia, J., Hulley, S., Herd, A., Khan, S., Newby, L. K., Waters, D., Vittinghoff, E., \& Wenger, N. (2002). Cardiovascular disease outcomes during 6.8 years of hormone therapy: Heart and Estrogen/progestin Replacement Study follow-up (HERS II). Journal of the American Medical Association, 288(1), 49-57. 


\section{Transdisciplinary Allostatic Load Review}

Grandjean, P., Weihe, P., Jorgensen, P. J., Clarkson, T., Cernichiari, E., \& Videro, T. (1992). Impact of maternal seafood diet on fetal exposure to mercury, selenium, and lead. Arch Environmental Health, 47(3), 185-195.

Gray, T. S., \& Bingaman, E. W. (1996). The amygdala: corticotropin-releasing factor, steroids, and stress. Critical Reviews in Neurobiology, 10(2), 155-168.

Green, M. F., Marder, S. R., Glynn, S. M., McGurk, S. R., Wirshing, W. C., Wirshing, D. A., Liberman, R. P., \& Mintz, J. (2002). The neurocognitive effects of low-dose haloperidol: a two-year comparison with risperidone. Biological Psychiatry, 51(12), 972-978.

Greendale, G. A., Kritz-Silverstein, D., Seeman, T., \& Barrett-Connor, E. (2000). Higher basal cortisol predicts verbal memory loss in postmenopausal women: Rancho Bernardo Study. Journal of the American Geriatric Society, 48(12), 1655-1658.

Greene, R. A. (2000). Estrogen and cerebral blood flow: a mechanism to explain the impact of estrogen on the incidence and treatment of Alzheimer's disease. Internation Journal of Fertility and Women's Medicine, 45(4), 253-257.

Gruenewald, T. L., Seeman, T. E., Karlamangla, A. S., \& Sarkisian, C. A. (2009). Allostatic load and frailty in older adults. Journal of the American Geriatric Society, 57(9), 1525-1531.

Gruenewald, T. L., Seeman, T. E., Ryff, C. D., Karlamangla, A. S., \& Singer, B. H. (2006). Combinations of biomarkers predictive of later life mortality. Proceeds of the National Academy of Sciences of the United States of America, 103(38), 14158-14163. 
Grundman, M., Jack, C. R., Jr., Petersen, R. C., Kim, H. T., Taylor, C., Datvian, M., Weiner, M. F., DeCarli, C., DeKosky, S. T., van Dyck, C., Darvesh, S., Yaffe, K., Kaye, J., Ferris, S. H., Thomas, R. G., \& Thal, L. J. (2003). Hippocampal volume is associated with memory but not monmemory cognitive performance in patients with mild cognitive impairment. Journal of Molecular Neuroscience, 20(3), 241-248.

Grundy, S. M., Brewer, H. B., Jr., Cleeman, J. I., Smith, S. C., Jr., \& Lenfant, C. (2004). Definition of metabolic syndrome: report of the National Heart, Lung, and Blood Institute/American Heart Association conference on scientific issues related to definition. Arteriosclerosis, Thrombosis, and Vascular Biology, 24(2), e13-18.

Gump, B. B., Reihman, J., Stewart, P., Lonky, E., Granger, D. A., \& Matthews, K. A. (2009). Blood lead $(\mathrm{Pb})$ levels: further evidence for an environmental mechanism explaining the association between socioeconomic status and psychophysiological dysregulation in children. Health Psychology, 28(5), 614-620.

Gump, B. B., Stewart, P., Reihman, J., Lonky, E., Darvill, T., Matthews, K. A., \& Parsons, P. J. (2005). Prenatal and early childhood blood lead levels and cardiovascular functioning in 9 1/2 year old children. Neurotoxicology and Teratology, 27(4), 655-665.

Gump, B. B., Stewart, P., Reihman, J., Lonky, E., Darvill, T., Parsons, P. J., \& Granger, D. A. (2008). Low-level prenatal and postnatal blood lead exposure and adrenocortical responses to acute stress in children. Environmental Health Perspectives, 116(2), 249-255. 
Gustafson, D., Rothenberg, E., Blennow, K., Steen, B., \& Skoog, I. (2003). An 18-year follow-up of overweight and risk of Alzheimer disease. Archives of Internal Medicine, 163(13), 1524-1528.

Haddad, P. M., \& Sharma, S. G. (2007). Adverse effects of atypical antipsychotics : differential risk and clinical implications. Central Nervous System Drugs, 21(11), 911-936.

Haley, W. E., Roth, D. L., Howard, G., \& Safford, M. M. Caregiving strain and estimated risk for stroke and coronary heart disease among spouse caregivers: differential effects by race and sex. Stroke, 41(2), 331-336.

Hamil-Luker, J., \& O'Rand, A. M. (2007). Gender differences in the link between childhood socioeconomic conditions and heart attack risk in adulthood. Demography, 44(1), 137-158.

Handa, R. J., Burgess, L. H., Kerr, J. E., \& O'Keefe, J. A. (1994a). Gonadal steroid hormone receptors and sex differences in the hypothalamo-pituitary-adrenal axis. Hormones and Behavior, 28(4), 464-476.

Handa, R. J., Nunley, K. M., Lorens, S. A., Louie, J. P., McGivern, R. F., \& Bollnow, M. R. (1994b). Androgen regulation of adrenocorticotropin and corticosterone secretion in the male rat following novelty and foot shock stressors. Physiology and Behavior, 55(1), 117124. 
Harman, S. M., Brinton, E. A., Cedars, M., Lobo, R., Manson, J. E., Merriam, G. R., Miller, V. M., Naftolin, F., \& Santoro, N. (2005a). KEEPS: The Kronos Early Estrogen Prevention Study. Climacteric: The Journal of the International Menopause Society, 8(1), 3-12.

Harman, S. M., Naftolin, F., Brinton, E. A., \& Judelson, D. R. (2005b). Is the estrogen controversy over? Deconstructing the Women's Health Initiative study: a critical evaluation of the evidence. Annals of the New York Academy of Science, 1052, 43-56.

Harvey, P. D., Meltzer, H., Simpson, G. M., Potkin, S. G., Loebel, A., Siu, C., \& Romano, S. J. (2004). Improvement in cognitive function following a switch to ziprasidone from conventional antipsychotics, olanzapine, or risperidone in outpatients with schizophrenia. Schizophrenia Research, 66(2-3), 101-113.

Heilmann, C., Grandjean, P., Weihe, P., Nielsen, F., \& Budtz-Jorgensen, E. (2006). Reduced antibody responses to vaccinations in children exposed to polychlorinated biphenyls. Public Library of Science Medicine, 3(8), e311.

Heim, C., \& Nemeroff, C. B. (2001). The role of childhood trauma in the neurobiology of mood and anxiety disorders: preclinical and clinical studies. Biological Psychiatry, 49(12), 10231039.

Heim, C., Newport, D. J., Heit, S., Graham, Y. P., Wilcox, M., Bonsall, R., Miller, A. H., \& Nemeroff, C. B. (2000). Pituitary-adrenal and autonomic responses to stress in women after sexual and physical abuse in childhood. Journal of the American Medical Association, 284(5), 592-597. 
Heim, C., Newport, D. J., Mletzko, T., Miller, A. H., \& Nemeroff, C. B. (2008). The link between childhood trauma and depression: insights from HPA axis studies in humans. Psychoneuroendocrinology, 33(6), 693-710.

Heim, C., Plotsky, P. M., \& Nemeroff, C. B. (2004). Importance of studying the contributions of early adverse experience to neurobiological findings in depression. Neuropsychopharmacology, 29(4), 641-648.

Hellhammer, J., Schlotz, W., Stone, A. A., Pirke, K. M., \& Hellhammer, D. (2004). Allostatic load, perceived stress, and health: a prospective study in two age groups. Annals of the New York Academy of Science, 1032, 8-13.

Hendrickx, H., McEwen, B. S., \& Ouderaa, F. (2005). Metabolism, mood and cognition in aging: the importance of lifestyle and dietary intervention. Neurobiology of Aging, 26 Suppl 1, 15.

Herman, J. P., Ostrander, M. M., Mueller, N. K., \& Figueiredo, H. (2005). Limbic system mechanisms of stress regulation: hypothalamo-pituitary-adrenocortical axis. Progress in Neuropsychopharmacology and Biological Psychiatry, 29(8), 1201-1213.

Hertzman, C. (1999). The biological embedding of early experience and its effects on health in adulthood. Annals of the New York Academy of Science, 896, 85-95. 


\section{Transdisciplinary Allostatic Load Review}

Hertzman, C., \& Wiens, M. (1996). Child development and long-term outcomes: a population health perspective and summary of successful interventions. Social Science and Medicine, 43(7), 1083-1095.

Ho, R. C., Niti, M., Yap, K. B., Kua, E. H., \& Ng, T. P. (2008). Metabolic syndrome and cognitive decline in chinese older adults: results from the singapore longitudinal ageing studies. American Joural of Geriatric Psychiatry, 16(6), 519-522.

Holmes, C., Cunningham, C., Zotova, E., Woolford, J., Dean, C., Kerr, S., Culliford, D., \& Perry, V. H. (2009). Systemic inflammation and disease progression in Alzheimer disease. Neurology, 73(10), 768-774.

Holsboer, F., \& Barden, N. (1996). Antidepressants and hypothalamic-pituitary-adrenocortical regulation. Endocrine Reviews, 17(2), 187-205.

Hontela, A., Dumont, P., Duclos, D., \& Fortin, R. (1995). Endocrine and Metabolic Dysfunction in Yellow Perch, Perca-Flavescens, Exposed to Organic Contaminants and Heavy-Metals in the St-Lawrence-River. Environmental Toxicology and Chemistry, 14(4), 725-731.

Horstmann, S., Dose, T., Lucae, S., Kloiber, S., Menke, A., Hennings, J., Spieler, D., Uhr, M., Holsboer, F., \& Ising, M. (2009). Suppressive effect of mirtazapine on the HPA system in acutely depressed women seems to be transient and not related to antidepressant action. Psychoneuroendocrinology, 34(2), 238-248. 
Hotchkiss, A. K., Rider, C. V., Blystone, C. R., Wilson, V. S., Hartig, P. C., Ankley, G. T., Foster, P. M., Gray, C. L., \& Gray, L. E. (2008). Fifteen years after "Wingspread"--environmental endocrine disrupters and human and wildlife health: where we are today and where we need to go. Toxicological Sciences, 105(2), 235-259.

Hoth, K. F., Haley, A. P., Gunstad, J., Paul, R. H., Poppas, A., Jefferson, A. L., Tate, D. F., Ono, M., Jerskey, B. A., \& Cohen, R. A. (2008). Elevated C-reactive protein is related to cognitive decline in older adults with cardiovascular disease. Journal of the American Geriatric Society, 56(10), 1898-1903.

Houston, B. K., \& Vavak, C. R. (1991). Cynical hostility: developmental factors, psychosocial correlates, and health behaviors. Health Psychology, 10(1), 9-17.

Howe, M. L., Toth, S. L., \& Cicchetti, D. (2006). Memory and Developmental Psychopathology. In D. Cicchetti \& D. Cohen (Eds.), Developmental Psychopathology Volume 2: Developmental Neuroscience (Vol. 2, pp. 629-656). New York: Wiley.

Huang, C. W., Lui, C. C., Chang, W. N., Lu, C. H., Wang, Y. L., \& Chang, C. C. (2009). Elevated basal cortisol level predicts lower hippocampal volume and cognitive decline in Alzheimer's disease. Journal of Clinical Neuroscience, 16(10), 1283-1286.

Hughes, G. C., \& Clark, E. A. (2007). Regulation of dendritic cells by female sex steroids: relevance to immunity and autoimmunity. Autoimmunity, 40(6), 470-481. 
Hughes, T. F., Borenstein, A. R., Schofield, E., Wu, Y., \& Larson, E. B. (2009). Association between late-life body mass index and dementia: The Kame Project. Neurology, 72(20), $1741-1746$.

Hunsberger, J., Austin, D. R., Henter, I. D., \& Chen, G. (2009). The neurotrophic and neuroprotective effects of psychotropic agents. Dialogues in Clinical Neuroscience, 11(3), 333-348.

Huurre, T., Junkkari, H., \& Aro, H. (2006). Long-term psychosocial effects of parental divorce: a follow-up study from adolescence to adulthood. European Archives of Psychiatry and Clinical Neuroscience, 256(4), 256-263.

ILO. (2000). Yearbook. Geneva: Internation Labour Office.

Ismail, K., Murray, R. M., Wheeler, M. J., \& O'Keane, V. (1998). The dexamethasone suppression test in schizophrenia. Psychological Medicine, 28(2), 311-317.

Jabbi, M., Kema, I. P., van der Pompe, G., te Meerman, G. J., Ormel, J., \& den Boer, J. A. (2007). Catechol-o-methyltransferase polymorphism and susceptibility to major depressive disorder modulates psychological stress response. Psychiatric Genetics, 17(3), 183-193.

Jack, C. R., Jr., Petersen, R. C., Xu, Y. C., O'Brien, P. C., Smith, G. E., Ivnik, R. J., Boeve, B. F., Waring, S. C., Tangalos, E. G., \& Kokmen, E. (1999). Prediction of AD with MRI-based hippocampal volume in mild cognitive impairment. Neurology, 52(7), 1397-1403. 
Jack, C. R., Jr., Petersen, R. C., Xu, Y. C., Waring, S. C., O'Brien, P. C., Tangalos, E. G., Smith, G. E., Ivnik, R. J., \& Kokmen, E. (1997). Medial temporal atrophy on MRI in normal aging and very mild Alzheimer's disease. [Research Support, Non-U.S. Gov't Research Support, U.S. Gov't, P.H.S.]. Neurology, 49(3), 786-794.

Jacobson, J. L., \& Jacobson, S. W. (1996). Intellectual impairment in children exposed to polychlorinated biphenyls in utero. New England Journal of Medicine, 335(11), 783-789.

Jagust, W., Gitcho, A., Sun, F., Kuczynski, B., Mungas, D., \& Haan, M. (2006). Brain imaging evidence of preclinical Alzheimer's disease in normal aging. Annals of Neurology, 59(4), 673-681.

Johansson, G., Huang, Q., \& Lindfors, P. (2007). A life-span perspective on women's careers, health, and well-being. Social Science and Medicine, 65(4), 685-697.

Johansson, M., Nilsson, S., \& Lund, B. O. (1998). Interactions between methylsulfonyl PCBs and the glucocorticoid receptor. Environmental Health Perspectives, 106(12), 769-772.

Jones, R. D., Pugh, P. J., Hall, J., Channer, K. S., \& Jones, T. H. (2003). Altered circulating hormone levels, endothelial function and vascular reactivity in the testicular feminised mouse. European Journal of Endocrinology, 148(1), 111-120.

Jones, R. L., Homa, D. M., Meyer, P. A., Brody, D. J., Caldwell, K. L., Pirkle, J. L., \& Brown, M. J. (2009). Trends in Blood Lead Levels and Blood Lead Testing Among US Children Aged 1 to 5 Years, 1988-2004. Pediatrics, 123(3), e376-e385. 
Jordanova, V., Stewart, R., Davies, E., Sherwood, R., \& Prince, M. (2007). Markers of inflammation and cognitive decline in an African-Caribbean population. International Journal of Geriatric Psychiatry, 22(10), 966-973.

Juster, R. P., McEwen, B. S., \& Lupien, S. J. (2010). Allostatic load biomarkers of chronic stress and impact on health and cognition. Neuroscience \& Biobehavioral Reviews, 35(1), 2-16.

Juster, R. P., Sindi, S., Marin, M. F., Perna, A., Hashemi, A., Pruessner, J. C., \& Lupien, S. J. (In Press). A clinical allostatic load index is associated with burnout symptoms and hypocortisolemic profiles in healthy workers. Psychoneuroendocrinology.

Jylha, M. (2009). What is self-rated health and why does it predict mortality? Towards a unified conceptual model. Social Science and Medicine.

Kajantie, E., \& Phillips, D. I. (2006). The effects of sex and hormonal status on the physiological response to acute psychosocial stress. Psychoneuroendocrinology, 31(2), 151-178.

Kalmijn, S., Foley, D., White, L., Burchfiel, C. M., Curb, J. D., Petrovitch, H., Ross, G. W., Havlik, R. J., \& Launer, L. J. (2000). Metabolic cardiovascular syndrome and risk of dementia in Japanese-American elderly men. The Honolulu-Asia aging study. Arteriosclerosis, Thrombosis and Vascular Biology, 20(10), 2255-2260.

Kalmijn, S., Launer, L. J., Stolk, R. P., de Jong, F. H., Pols, H. A., Hofman, A., Breteler, M. M., \& Lamberts, S. W. (1998). A prospective study on cortisol, dehydroepiandrosterone sulfate, 


\section{Transdisciplinary Allostatic Load Review}

and cognitive function in the elderly. Journal of Clinical Endocrinology and Metabolism, 83(10), 3487-3492.

Kapczinski, F., Vieta, E., Andreazza, A. C., Frey, B. N., Gomes, F. A., Tramontina, J., KauerSant'anna, M., Grassi-Oliveira, R., \& Post, R. M. (2008). Allostatic load in bipolar disorder: implications for pathophysiology and treatment. Neuroscience and Biobehavioral Reviews, 32(4), 675-692.

Karl, A., Schaefer, M., Malta, L. S., Dorfel, D., Rohleder, N., \& Werner, A. (2006). A metaanalysis of structural brain abnormalities in PTSD. Neuroscience and Biobehavioral Reviews, 30(7), 1004-1031.

Karlamangla, A. S., Singer, B. H., Chodosh, J., McEwen, B. S., \& Seeman, T. E. (2005a). Urinary cortisol excretion as a predictor of incident cognitive impairment. Neurobiology of Aging, 26 Suppl 1, 80-84.

Karlamangla, A. S., Singer, B. H., Greendale, G. A., \& Seeman, T. E. (2005b). Increase in epinephrine excretion is associated with cognitive decline in elderly men: MacArthur studies of successful aging. Psychoneuroendocrinology, 30(5), 453-460.

Karlamangla, A. S., Singer, B. H., McEwen, B. S., Rowe, J. W., \& Seeman, T. E. (2002). Allostatic load as a predictor of functional decline. MacArthur studies of successful aging. Journal of Clinical Epidemiology, 55(7), 696-710. 
Karlamangla, A. S., Singer, B. H., \& Seeman, T. E. (2006). Reduction in allostatic load in older adults is associated with lower all-cause mortality risk: MacArthur studies of successful aging. Psychosomatic Medicine, 68(3), 500-507.

Kelly, S. J., Young, R., Sweeting, H., Fischer, J. E., \& West, P. (2008). Levels and confounders of morning cortisol collected from adolescents in a naturalistic (school) setting. Psychoneuroendocrinology, 33(9), 1257-1268.

Kendler, K. S., Kuhn, J. W., Vittum, J., Prescott, C. A., \& Riley, B. (2005). The interaction of stressful life events and a serotonin transporter polymorphism in the prediction of episodes of major depression: a replication. Archives of General Psychiatry, 62(5), 529-535.

Kerr, L. K., \& Kerr, L. D., Jr. (2001). Screening tools for depression in primary care: the effects of culture, gender, and somatic symptoms on the detection of depression. Western Journal of Medicine, 175(5), 349-352.

Kessel, F., \& Rosenfield, P. L. (2008). Toward transdisciplinary research: historical and contemporary perspectives. American Journal of Preventative Medicine, 35(2 Suppl), S225-234.

Kessel, F., Rosenfield, P. L., \& Anderson, N. B. (2008). Interdisciplinary Research. New York: Oxford University Press. 
Kiecolt-Glaser, J. K., Dura, J. R., Speicher, C. E., Trask, O. J., \& Glaser, R. (1991). Spousal caregivers of dementia victims: longitudinal changes in immunity and health. Psychosomatic Medicine, 53(4), 345-362.

Kim, J. M., Stewart, R., Kim, S. W., Shin, I. S., Yang, S. J., \& Yoon, J. S. (2009). Cholesterol and serotonin transporter polymorphism interactions in late-life depression. Neurobiology of Aging, 32(2), 336-343.

Kim, S. H., Han, D. H., Joo, S. Y., \& Min, K. J. (2010). The effect of dopamine partial agonists on the nicotine dependency in patients with schizophrenia. Human Psychopharmacology, 25(2), 187-190.

Kim, Y. K., Jung, H. G., Myint, A. M., Kim, H., \& Park, S. H. (2007). Imbalance between proinflammatory and anti-inflammatory cytokines in bipolar disorder. Journal of Affective Disorders, 104(1-3), 91-95.

King, A. C., Oka, R.K., Young, D.R. (1992). Ambulatory blood pressure and heart rate responses to the stress of work and caregiving in older women. The Journals of Gerontology. Series A, Biological Sciences and Medical Sciences, 49, 239-245.

Kinnunen, M. J., Kaprio, J., \& Pulkkinen, L. (2005). Allostatic load of men and women in early middle age. Journal of Individual Differences, 26(1), 20-28. 


\section{Transdisciplinary Allostatic Load Review}

Kirschbaum, C., Klauer, T., Filipp, S. H., \& Hellhammer, D. H. (1995). Sex-specific effects of social support on cortisol and subjective responses to acute psychological stress. Psychosomatic Medicine, 57(1), 23-31.

Kirschbaum, C., Kudielka, B. M., Gaab, J., Schommer, N. C., \& Hellhammer, D. H. (1999). Impact of gender, menstrual cycle phase, and oral contraceptives on the activity of the hypothalamus-pituitary-adrenal axis. Psychosomatic Medicine, 61(2), 154-162.

Kirschbaum, C., Pirke, K. M., \& Hellhammer, D. H. (1995). Preliminary evidence for reduced cortisol responsivity to psychological stress in women using oral contraceptive medication. Psychoneuroendocrinology, 20(5), 509-514.

Kirschbaum, C., Wust, S., Faig, H. G., \& Hellhammer, D. H. (1992). Heritability of cortisol responses to human corticotropin-releasing hormone, ergometry, and psychological stress in humans. Journal of Clinical Endocrinology and Metabolism, 75(6), 1526-1530.

Kirschbaum, C., Wust, S., \& Hellhammer, D. (1992). Consistent sex differences in cortisol responses to psychological stress. Psychosomatic Medicine, 54(6), 648-657.

Kivipelto, M., Helkala, E. L., Laakso, M. P., Hanninen, T., Hallikainen, M., Alhainen, K., Soininen, H., Tuomilehto, J., \& Nissinen, A. (2001). Midlife vascular risk factors and Alzheimer's disease in later life: longitudinal, population based study. British Medical Journal, 322(7300), 1447-1451. 
Kivipelto, M., Ngandu, T., Fratiglioni, L., Viitanen, M., Kareholt, I., Winblad, B., Helkala, E. L., Tuomilehto, J., Soininen, H., \& Nissinen, A. (2005). Obesity and vascular risk factors at midlife and the risk of dementia and Alzheimer disease. Archives of Neurology, 62(10), 1556- 1560.

Kivipelto, M., Ngandu, T., Laatikainen, T., Winblad, B., Soininen, H., \& Tuomilehto, J. (2006). Risk score for the prediction of dementia risk in 20 years among middle aged people: a longitudinal, population-based study. Lancet Neurology, 5(9), 735-741.

Knutson, K. L., Spiegel, K., Penev, P., \& Van Cauter, E. (2007). The metabolic consequences of sleep deprivation. Sleep Medicine Reviews, 11(3), 163-178.

Knutson, K. L., Van Cauter, E., Rathouz, P. J., Yan, L. L., Hulley, S. B., Liu, K., \& Lauderdale, D. S. (2009). Association between sleep and blood pressure in midlife: the CARDIA sleep study. Archives of Internal Medicine, 169(11), 1055-1061.

Kolsch, H., Jessen, F., Freymann, N., Kreis, M., Hentschel, F., Maier, W., \& Heun, R. (2005). ACE I/D polymorphism is a risk factor of Alzheimer's disease but not of vascular dementia. Neuroscience Letters, 377(1), 37-39.

Komesaroff, P. A., Esler, M. D., \& Sudhir, K. (1999). Estrogen supplementation attenuates glucocorticoid and catecholamine responses to mental stress in perimenopausal women. Journal of Clinical Endocrinology and Metabolism, 84(2), 606-610. 
Koob, G. F. (2003). Alcoholism: allostasis and beyond. Alcoholism, Clinical and Experimental Research, 27(2), 232-243.

Koob, G. F. (2008). A role for brain stress systems in addiction. Neuron, 59(1), 11-34.

Koob, G. F. (2009). Neurobiological substrates for the dark side of compulsivity in addiction. Neuropharmacology, 56 Suppl 1, 18-31.

Koob, G. F., Ahmed, S. H., Boutrel, B., Chen, S. A., Kenny, P. J., Markou, A., O'Dell, L. E., Parsons, L. H., \& Sanna, P. P. (2004). Neurobiological mechanisms in the transition from drug use to drug dependence. Neuroscience and Biobehavioral Reviews, 27(8), 739-749.

Koob, G. F., \& Le Moal, M. (2001). Drug addiction, dysregulation of reward, and allostasis. Neuropsychopharmacology, 24(2), 97-129.

Koob, G. F., \& Le Moal, M. (2008a). Addiction and the brain antireward system. Annual Review of Psychology, 59, 29-53.

Koob, G. F., \& Le Moal, M. (2008b). Review. Neurobiological mechanisms for opponent motivational processes in addiction. Philosophical Transactions of the Royal Society of London. Series B, Biological Sciences, 363(1507), 3113-3123.

Korf, E. S., Wahlund, L. O., Visser, P. J., \& Scheltens, P. (2004). Medial temporal lobe atrophy on MRI predicts dementia in patients with mild cognitive impairment. Neurology, 63(1), 94100. 
Kornstein, S. G., Schatzberg, A. F., Thase, M. E., Yonkers, K. A., McCullough, J. P., Keitner, G. I., Gelenberg, A. J., Ryan, C. E., Hess, A. L., Harrison, W., Davis, S. M., \& Keller, M. B. (2000). Gender differences in chronic major and double depression. Journal of Affective Disorders, 60(1), 1-11.

Korte, S. M., Koolhaas, J. M., Wingfield, J. C., \& McEwen, B. S. (2005). The Darwinian concept of stress: benefits of allostasis and costs of allostatic load and the trade-offs in health and disease. Neuroscience and Biobehavioral Reviews, 29(1), 3-38.

Kramer, A. F., Hahn, S., Cohen, N. J., Banich, M. T., McAuley, E., Harrison, C. R., Chason, J., Vakil, E., Bardell, L., Boileau, R. A., \& Colcombe, A. (1999). Ageing, fitness and neurocognitive function. Nature, 400(6743), 418-419.

Kramer, B. J. (1997). Gain in the caregiving experience: where are we? What next? Gerontologist, $37(2), 218-232$.

Kroeze, W. K., Hufeisen, S. J., Popadak, B. A., Renock, S. M., Steinberg, S., Ernsberger, P., Jayathilake, K., Meltzer, H. Y., \& Roth, B. L. (2003). H1-histamine receptor affinity predicts short-term weight gain for typical and atypical antipsychotic drugs. Neuropsychopharmacology, 28(3), 519-526.

Kronenberg, F. (1990). Hot flashes: epidemiology and physiology. Annals of the New York Academy of Science, 592, 52-86; discussion 123-133. 
Kropp, S., Kern, V., Lange, K., Degner, D., Hajak, G., Kornhuber, J., Ruther, E., Emrich, H. M., Schneider, U., \& Bleich, S. (2005). Oxidative stress during treatment with first- and second-generation antipsychotics. Journal of Neuropsychiatry and Clinical Neuroscience, $17(2), 227-231$.

Krystal, J. H., D'Souza, D. C., Gallinat, J., Driesen, N., Abi-Dargham, A., Petrakis, I., Heinz, A., \& Pearlson, G. (2006). The vulnerability to alcohol and substance abuse in individuals diagnosed with schizophrenia. Neurotoxicological Research, 10(3-4), 235-252.

Kudielka, B. M., \& Kirschbaum, C. (2005). Sex differences in HPA axis responses to stress: a review. Biological Psychology, 69(1), 113-132.

Lagerlof, E. (2005). Global gender issues in relation to health and industrial work. Geneva: World Health Organization.

Lam, L. T. (2008). Sleep disruptions and mental health of primary caregivers of persons with disability due to chronic mental and physical conditions in the Australian population. Australian e-Journal for the Advacement of Mental Health, 7(3).

Landrigan, P. J. (1999). Risk assessment for children and other sensitive populations. Annals of the New York Acedemy of Science, 895, 1-9.

Langelaan, S., Bakker, A. B., Schaufeli, W. B., van Rhenen, W., \& van Doornen, L. J. (2007). Is burnout related to allostatic load? International Journal of Behavioral Medicine, 14(4), 213-221. 


\section{Transdisciplinary Allostatic Load Review}

Laurin, D., David Curb, J., Masaki, K. H., White, L. R., \& Launer, L. J. (2009). Midlife C-reactive protein and risk of cognitive decline: a 31-year follow-up. Neurobiology of Aging, 30(11), 1724-1727.

Lee, B. K., Glass, T. A., McAtee, M. J., Wand, G. S., Bandeen-Roche, K., Bolla, K. I., \& Schwartz, B. S. (2007a). Associations of salivary cortisol with cognitive function in the Baltimore memory study. Archives of General Psychiatry, 64(7), 810-818.

Lee, D. H., Lee, I. K., Porta, M., Steffes, M., \& Jacobs, D. R., Jr. (2007b). Relationship between serum concentrations of persistent organic pollutants and the prevalence of metabolic syndrome among non-diabetic adults: results from the National Health and Nutrition Examination Survey 1999-2002. Diabetologia, 50(9), 1841-1851.

Lee, S., Colditz, G. A., Berkman, L. F., \& Kawachi, I. (2003). Caregiving and risk of coronary heart disease in U.S. women: a prospective study. American Journal of Preventative Medicine, 24(2), 113-119.

Lee, S., Kawachi, I., \& Grodstein, F. (2004). Does caregiving stress affect cognitive function in older women? Journal of Nervous and Mental Disorders, 192(1), 51-57.

Lee, Z. S., Chan, J. C., Yeung, V. T., Chow, C. C., Lau, M. S., Ko, G. T., Li, J. K., Cockram, C. S., \& Critchley, J. A. (1999). Plasma insulin, growth hormone, cortisol, and central obesity among young Chinese type 2 diabetic patients. Diabetes Care, 22(9), 1450-1457. 
Levin, E. D., Wilson, W., Rose, J. E., \& McEvoy, J. (1996). Nicotine-haloperidol interactions and cognitive performance in schizophrenics. Neuropsychopharmacology, 15(5), 429-436.

Levine, S. (2005). Developmental determinants of sensitivity and resistance to stress. Psychoneuroendocrinology, 30(10), 939-946.

Levine, S., \& Ursin, H. (1991). What is stress? . In M. R. Brown, G. F. Koob \& C. Rivier (Eds.), Stress Neurobiology and Neuroendocrinology (pp. 3-21). New York: Marcel Dekker Inc.

Li, G., Cherrier, M. M., Tsuang, D. W., Petrie, E. C., Colasurdo, E. A., Craft, S., Schellenberg, G. D., Peskind, E. R., Raskind, M. A., \& Wilkinson, C. W. (2006). Salivary cortisol and memory function in human aging. Neurobiology of Aging, 27(11), 1705-1714.

Li, G., Shofer, J. B., Kukull, W. A., Peskind, E. R., Tsuang, D. W., Breitner, J. C., McCormick, W., Bowen, J. D., Teri, L., Schellenberg, G. D., \& Larson, E. B. (2005). Serum cholesterol and risk of Alzheimer disease: a community-based cohort study. Neurology, 65(7), 10451050.

Li, L. A., \& Wang, P. W. (2005). PCB126 induces differential changes in androgen, cortisol, and aldosterone biosynthesis in human adrenocortical H295R cells. Toxicological Science, $85(1), 530-540$.

Li, W., Zhang, J. Q., Sun, J., Ke, J. H., Dong, Z. Y., \& Wang, S. (2007). Job stress related to glyco-lipid allostatic load, adiponectin and visfatin. Stress and Health, 23, 257-266. 
Lieberman, J. A., Bymaster, F. P., Meltzer, H. Y., Deutch, A. Y., Duncan, G. E., Marx, C. E., Aprille, J. R., Dwyer, D. S., Li, X. M., Mahadik, S. P., Duman, R. S., Porter, J. H., Modica-Napolitano, J. S., Newton, S. S., \& Csernansky, J. G. (2008). Antipsychotic drugs: comparison in animal models of efficacy, neurotransmitter regulation, and neuroprotection. Pharmacological Reviews, 60(3), 358-403.

Lieberman, J. A., Tollefson, G. D., Charles, C., Zipursky, R., Sharma, T., Kahn, R. S., Keefe, R. S., Green, A. I., Gur, R. E., McEvoy, J., Perkins, D., Hamer, R. M., Gu, H., \& Tohen, M. (2005). Antipsychotic drug effects on brain morphology in first-episode psychosis. Archives of General Psychiatry, 62(4), 361-370.

Lin, Y. S., Rathod, D., Ho, W. C., \& Caffrey, J. J. (2009). Cadmium exposure is associated with elevated blood C-reactive protein and fibrinogen in the U.S. population: the third national health and nutrition examination survey (NHANES III, 1988-1994). Annals of Epidemiology, 19(8), 592-596.

Lind, P. M., Orberg, J., Edlund, U. B., Sjoblom, L., \& Lind, L. (2004). The dioxin-like pollutant PCB 126 (3,3',4,4',5-pentachlorobiphenyl) affects risk factors for cardiovascular disease in female rats. Toxicology Letters, 150(3), 293-299.

Lindfors, P., Lundberg, O., \& Lundberg, U. (2006). Allostatic load and clinical risk as related to sense of coherence in middle-aged women. Psychosomatic Medicine, 68(5), 801-807.

Linkowski, P., Kerkhofs, M., Van Onderbergen, A., Hubain, P., Copinschi, G., L'HermiteBaleriaux, M., Leclercq, R., Brasseur, M., Mendlewicz, J., \& Van Cauter, E. (1994). The 
24-hour profiles of cortisol, prolactin, and growth hormone secretion in mania. Archives of General Psychiatry, 51(8), 616-624.

Linkowski, P., Van Onderbergen, A., Kerkhofs, M., Bosson, D., Mendlewicz, J., \& Van Cauter, E. (1993). Twin study of the 24-h cortisol profile: evidence for genetic control of the human circadian clock. American Journal of Physiology, 264(2 Pt 1), E173-181.

Lips, H. (2008). Sex \& Gender: an introduction (6th ed.). New-York: McGraw-Hill Higher Education.

Liu, H. C., Yang, Y. Y., Chou, Y. M., Chen, K. P., Shen, W. W., \& Leu, S. J. (2004). Immunologic variables in acute mania of bipolar disorder. Journal of Neuroimmunology, $150(1-2), 116-122$.

Lord, C., Buss, C., Lupien, S. J., \& Pruessner, J. C. (2008). Hippocampal volumes are larger in postmenopausal women using estrogen therapy compared to past users, never users and men: a possible window of opportunity effect. Neurobiology of Aging, 29(1), 95-101.

Loucks, E. B., Juster, R. P., \& Pruessner, J. C. (2008). Neuroendocrine biomarkers, allostatic load, and the challenge of measurement: A commentary on Gersten. Social Science and Medicine, 66, 525-530.

Love, O. P., Shutt, L. J., Silfies, J. S., Bortolotti, G. R., Smits, J. E. G., \& Bird, D. M. (2003). Effects of dietary PCB exposure on adrenocortical function in captive American kestrels (Falco sparverius). Ecotoxicology, 12(1-4), 199-208. 
Low, A. K., Russell, L. D., Holman, H. E., Shepherd, J. M., Hicks, G. S., \& Brown, C. A. (2002). Hormone replacement therapy and coronary heart disease in women: a review of the evidence. American Journal of Medical Science, 324(4), 180-184.

Lu, Y. F., \& Wykle, M. (2007). Relationships between caregiver stress and self-care behaviors in response to symptoms. Clinical Nursing Research, 16(1), 29-43.

Lublin, H., Eberhard, J., \& Levander, S. (2005). Current therapy issues and unmet clinical needs in the treatment of schizophrenia: a review of the new generation antipsychotics. International Journal of Clinical Psychopharmacology, 20(4), 183-198.

Luchsinger, J. A., Reitz, C., Honig, L. S., Tang, M. X., Shea, S., \& Mayeux, R. (2005). Aggregation of vascular risk factors and risk of incident Alzheimer disease. Neurology, $65(4), 545-551$.

Lupien, S., Lecours, A. R., Lussier, I., Schwartz, G., Nair, N. P., \& Meaney, M. J. (1994). Basal cortisol levels and cognitive deficits in human aging. Journal of Neuroscience, 14(5 Pt 1), 2893-2903.

Lupien, S. J., de Leon, M., de Santi, S., Convit, A., Tarshish, C., Nair, N. P., Thakur, M., McEwen, B. S., Hauger, R. L., \& Meaney, M. J. (1998). Cortisol levels during human aging predict hippocampal atrophy and memory deficits. Nature Neuroscience, 1(1), 6973. 


\section{Transdisciplinary Allostatic Load Review}

Lupien, S. J., Evans, A., Lord, C., Miles, J., Pruessner, M., Pike, B., \& Pruessner, J. C. (2007). Hippocampal volume is as variable in young as in older adults: implications for the notion of hippocampal atrophy in humans. Neuroimage, 34(2), 479-485.

Lupien, S. J., Fiocco, A., Wan, N., Maheu, F., Lord, C., Schramek, T., \& Tu, M. T. (2005). Stress hormones and human memory function across the lifespan. Psychoneuroendocrinology, $30(3), 225-242$.

Lupien, S. J., King, S., Meaney, M. J., \& McEwen, B. S. (2000). Child's stress hormone levels correlate with mother's socioeconomic status and depressive state. Biological Psychiatry, 48(10), 976-980.

Lupien, S. J., King, S., Meaney, M. J., \& McEwen, B. S. (2001). Can poverty get under your skin? Basal cortisol levels and cognitive function in children from low and high socioeconomic status. Development and Psychopathology, 13(03), 653-676.

Lupien, S. J., McEwen, B. S., Gunnar, M. R., \& Heim, C. (2009). Effects of stress throughout the lifespan on the brain, behaviour and cognition. Nature Reviews. Neuroscience, 10(6), 434445.

Lupien, S. J., Ouellet-Morin, I., Hupbach, A., Walker, D., Tu, M. T., Buss, C., Pruessner, J., \& McEwen, B. (2006). Beyond the stress concept: allostatic load - a developmental biological and cognitive perspective. In D. Cicchetti (Ed.), Handbook Series on Developmental Psychopathology (pp. 784-809). 
MacLennan, A. H., Henderson, V. W., Paine, B. J., Mathias, J., Ramsay, E. N., Ryan, P., Stocks, N. P., \& Taylor, A. W. (2006). Hormone therapy, timing of initiation, and cognition in women aged older than 60 years: the REMEMBER pilot study. Menopause, 13(1), 28-36.

MacQueen, G. M., Campbell, S., McEwen, B. S., MacDonald, K., Amano, S., Joffe, R. T., Nahmias, C., \& Young, L. T. (2003). Course of illness, hippocampal function, and hippocampal volume in major depression. Proceedings of the National Academy of Sciences of the United States of America, 100(3), 1387-1392.

Maes, M., Meltzer, H. Y., \& Bosmans, E. (1994). Immune-inflammatory markers in schizophrenia: comparison to normal controls and effects of clozapine. Acta Psychiatrica Scandinavica, 89(5), 346-351.

Maggio, M., Basaria, S., Ceda, G. P., Ble, A., Ling, S. M., Bandinelli, S., Valenti, G., \& Ferrucci, L. (2005). The relationship between testosterone and molecular markers of inflammation in older men. Journal of Endocrinological Investigation, 28(11 Suppl Proceedings), 116119.

Maki, P. M., \& Resnick, S. M. (2000). Longitudinal effects of estrogen replacement therapy on PET cerebral blood flow and cognition. Neurobioogy of Aging, 21(2), 373-383.

Malik, R. A., Williamson, S., Abbott, C., Carrington, A. L., Iqbal, J., Schady, W., \& Boulton, A. J. (1998). Effect of angiotensin-converting-enzyme (ACE) inhibitor trandolapril on human diabetic neuropathy: randomised double-blind controlled trial. Lancet, 352(9145), 19781981. 
Maloney, E. M., Boneva, R., Nater, U. M., \& Reeves, W. C. (2009). Chronic Fatigue Syndrome and High Allostatic Load: Results From a Population-Based Case-Control Study in Georgia. Psychosomatic Medicine, 71(5) 549-556.

Maloney, E. M., Gurbaxani, B. M., Jones, J. F., de Souza Coelho, L., Pennachin, C., \& Goertzel, B. N. (2006). Chronic fatigue syndrome and high allostatic load. Pharmacogenomics, 7(3), 467-473.

Maninger, N., Wolkowitz, O. M., Reus, V. I., Epel, E. S., \& Mellon, S. H. (2009). Neurobiological and neuropsychiatric effects of dehydroepiandrosterone (DHEA) and DHEA sulfate (DHEAS). Frontiers in Neuroendocrinology, 30(1), 65-91.

Mann, K., Rossbach, W., Muller, M. J., Muller-Siecheneder, F., Pott, T., Linde, I., Dittmann, R. W., \& Hiemke, C. (2006). Nocturnal hormone profiles in patients with schizophrenia treated with olanzapine. Psychoneuroendocrinology, 31(2), 256-264.

Mariani, E., Polidori, M. C., Cherubini, A., \& Mecocci, P. (2005). Oxidative stress in brain aging, neurodegenerative and vascular diseases: an overview. Journal of Chromatography. Analytical Technologies in the Biomedical and Life Sciences, 827(1), 65-75.

Marin, P., Holmang, S., Jonsson, L., Sjostrom, L., Kvist, H., Holm, G., Lindstedt, G., \& Bjorntorp, P. (1992a). The effects of testosterone treatment on body composition and metabolism in middle-aged obese men. International Journal of Obesity and Related Metabolic Disorders: Journal of the International Association for the Study of Obesity, 16(12), 991-997. 


\section{Transdisciplinary Allostatic Load Review}

Marin, P., Krotkiewski, M., \& Bjorntorp, P. (1992b). Androgen treatment of middle-aged, obese men: effects on metabolism, muscle and adipose tissues. European Journal of Medicine, $1(6), 329-336$.

Marinari, K. T., Leshner, A. I., \& Doyle, M. P. (1976). Menstrual cycle status and adrenocortical reactivity to psychological stress. Psychoneuroendocrinology, 1(3), 213-218.

Marinelli, M., Rudick, C. N., Hu, X. T., \& White, F. J. (2006). Excitability of dopamine neurons: modulation and physiological consequences. CNS and Neurological Disorders Drug Targets, 5(1), 79-97.

Marioni, R. E., Stewart, M. C., Murray, G. D., Deary, I. J., Fowkes, F. G., Lowe, G. D., Rumley, A., \& Price, J. F. (2009). Peripheral levels of fibrinogen, C-reactive protein, and plasma viscosity predict future cognitive decline in individuals without dementia. Psychosomatic Medicine, 71(8), 901-906.

Markianos, M., Hatzimanolis, J., \& Lykouras, L. (1999). Switch from neuroleptics to clozapine does not influence pituitary-gonadal axis hormone levels in male schizophrenic patients. European Neuropsychopharmacology, 9(6), 533-536.

Maschio, G., Alberti, D., Janin, G., Locatelli, F., Mann, J. F., Motolese, M., Ponticelli, C., Ritz, E., \& Zucchelli, P. (1996). Effect of the angiotensin-converting-enzyme inhibitor benazepril on the progression of chronic renal insufficiency. The Angiotensin-ConvertingEnzyme Inhibition in Progressive Renal Insufficiency Study Group. New England Journal of Medicine, 334(15), 939-945. 
Maselko, J., Kubzansky, L., Kawachi, I., Seeman, T., \& Berkman, L. (2007). Religious service attendance and allostatic load among high-functioning elderly. Psychosomatic Medicine, $69(5), 464-472$.

Mason, J. W. (1968). A review of psychoendocrine research on the sympathetic-adrenal medullary system. Psychosomatic Medicine, 30(5), Suppl:631-653.

Matsuda, O., Hasebe, N., Ikehara, K., Futatsuya, M., \& Akahane, N. (1997). Longitudinal study of the mental health of caregivers caring for elderly patients with dementia: effect of institutional placement on mental health. Psychiatry and Clinical Neuroscience, 51(5), 289-293.

Maxwell, S. R. (1998). Women and heart disease. Basic Research in Cardiology, 93 Suppl 2, 7984.

McAllister-Williams, R. H., Ferrier, I. N., \& Young, A. H. (1998). Mood and neuropsychological function in depression: the role of corticosteroids and serotonin. Psychological Medicine, $28(3), 573-584$.

McClain, C. S., Rosenfeld, B., \& Breitbart, W. (2003). Effect of spiritual well-being on end-of-life despair in terminally-ill cancer patients. Lancet, 361(9369), 1603-1607.

McEvoy, J., Freudenreich, O., McGee, M., VanderZwaag, C., Levin, E., \& Rose, J. (1995). Clozapine decreases smoking in patients with chronic schizophrenia. Biological Psychiatry, 37(8), 550-552. 
McEwen, B. S. (1998a). Protective and damaging effects of stress mediators. New England Journal of Medicine, 338(3), 171-179.

McEwen, B. S. (1998b). Stress, adaptation, and disease. Allostasis and allostatic load. Annals of the New York Academy of Science, 840, 33-44.

McEwen, B. S. (2000a). Allostasis and allostatic load: implications for neuropsychopharmacology. Neuropsychopharmacology, 22(2), 108-124.

McEwen, B. S. (2000b). The neurobiology of stress: from serendipity to clinical relevance. Brain Reearchs, 886(1-2), 172-189.

McEwen, B. S. (2001). Plasticity of the hippocampus: adaptation to chronic stress and allostatic load. Annals of the New York Academy of Science, 933, 265-277.

McEwen, B. S. (2002a). Estrogen actions throughout the brain. Recent Progress in Hormone Research, 57, 357-384.

McEwen, B. S. (2002b). The neurobiology and neuroendocrinology of stress. Implications for post-traumatic stress disorder from a basic science perspective. Psychiatric Clinics of North America, 25(2), 469-494.

McEwen, B. S. (2002c). Sex, stress and the hippocampus: allostasis, allostatic load and the aging process. Neurobiology of Aging, 23(5), 921-939. 
McEwen, B. S. (2003a). Early life influences on life-long patterns of behavior and health. Mental Retardation and Developmental Disabilities Research Reviews, 9, 149-154.

McEwen, B. S. (2003b). Interacting mediators of allostasis and allostatic load: towards an understanding of resilience in aging. Metabolism, 52(10 Suppl 2), 10-16.

McEwen, B. S. (2003c). Mood disorders and allostatic load. Biological Psychiatry, 54(3), 200207.

McEwen, B. S. (2004). Protection and damage from acute and chronic stress: allostasis and allostatic overload and relevance to the pathophysiology of psychiatric disorders. Annals of the New York Academy of Science, 1032, 1-7.

McEwen, B. S. (2005). Glucocorticoids, depression, and mood disorders: structural remodeling in the brain. Metabolism, 54(5 Suppl 1), 20-23.

McEwen, B. S. (2006a). Protective and damaging effects of stress mediators: central role of the brain. Dialogues in Clinical Neuroscience, 8(4), 367-381.

McEwen, B. S. (2006b). Sleep deprivation as a neurobiologic and physiologic stressor: Allostasis and allostatic load. Metabolism, 55(10 Suppl 2), S20-23.

McEwen, B. S. (2007a). Physiology and neurobiology of stress and adaptation: central role of the brain. Physiological Reviews, 87(3), 873-904. 
McEwen, B. S. (2008). Central effects of stress hormones in health and disease: Understanding the protective and damaging effects of stress and stress mediators. European Journal of Pharmacology, 583(2-3), 174-185.

McEwen, B. S., de Leon, M. J., Lupien, S. J., \& Meaney, M. J. (1999). Corticosteroids, the Aging Brain and Cognition. Trends in Endocrinology and Metabolism, 10(3), 92-96.

McEwen, B. S., \& Gianaros, P. J. (2010). Central role of the brain in stress and adaptation: links to socioeconomic status, health, and disease. Annals of the New York Academy of Science, $1186,190-222$.

McEwen, B. S., \& Seeman, T. (1999). Protective and damaging effects of mediators of stress. Elaborating and testing the concepts of allostasis and allostatic load. Annals of the New York Academy of Science, 896, 30-47.

McEwen, B. S., \& Stellar, E. (1993). Stress and the individual. Mechanisms leading to disease. Archives of Internal Medicine, 153(18), 2093-2101.

McEwen, B. S., Weiss, J. M., \& Schwartz, L. S. (1968). Selective retention of corticosterone by limbic structures in rat brain. Nature, 220(5170), 911-912.

McEwen, B. S., \& Wingfield, J. C. (2003). The concept of allostasis in biology and biomedicine. Hormones and Behavior, 43(1), 2-15. 
McGowan, P. O., Sasaki, A., D'Alessio, A. C., Dymov, S., Labonte, B., Szyf, M., Turecki, G., \& Meaney, M. J. (2009). Epigenetic regulation of the glucocorticoid receptor in human brain associates with childhood abuse. Nature Neuroscience, 12(3), 342-348.

McKhann, G., Drachman, D., Folstein, M., Katzman, R., Price, D., \& Stadlan, E. M. (1984). Clinical diagnosis of Alzheimer's disease: report of the NINCDS-ADRDA Work Group under the auspices of Department of Health and Human Services Task Force on Alzheimer's Disease. Neurology, 34(7), 939-944.

McKibbin, C. L., Ancoli-Israel, S., Dimsdale, J., Archuleta, C., von Kanel, R., Mills, P., Patterson, T. L., \& Grant, I. (2005). Sleep in spousal caregivers of people with Alzheimer's disease. Sleep, 28(10), 1245-1250.

Mealy, K., Robinson, B., Millette, C. F., Majzoub, J., \& Wilmore, D. W. (1990). The testicular effects of tumor necrosis factor. Annals of Surgery, 211(4), 470-475.

Meaney, M. J., Aitken, D. H., van Berkel, C., Bhatnagar, S., \& Sapolsky, R. M. (1988). Effect of neonatal handling on age-related impairments associated with the hippocampus. Science, 239(4841 Pt 1), 766-768.

Meaney, M. J., \& Szyf, M. (2005). Environmental programming of stress responses through DNA methylation: life at the interface between a dynamic environment and a fixed genome. Dialogues in Clinical Neuroscience, 7(2), 103-123. 
Meltzer, L. J., \& Mindell, J. A. (2006). Impact of a child's chronic illness on maternal sleep and daytime functioning. Archives of Internal Medicine, 166(16), 1749-1755.

Menke, A., Muntner, P., Batuman, V., Silbergeld, E. K., \& Guallar, E. (2006). Blood lead below $0.48 \mathrm{mu} \mathrm{mol} / \mathrm{L}$ (10 mu g/dL) and mortality among US adults. Circulation, 114(13), 13881394.

Meserve, L. A., Murray, B. A., \& Landis, J. A. (1992). Influence of maternal ingestion of Aroclor 1254 (PCB) or FireMaster BP-6 (PBB) on unstimulated and stimulated corticosterone levels in young rats. Bulletin of Environmental Contamination and Toxicology, 48(5), 715720.

Mezzich, J. E. (2010). World Psychiatric Association perspectives on person-centered psychiatry and medicine. Internation Journal of Integrated Care, 10 Suppl, e003.

Mielke, M. M., Rosenberg, P. B., Tschanz, J., Cook, L., Corcoran, C., Hayden, K. M., Norton, M., Rabins, P. V., Green, R. C., Welsh-Bohmer, K. A., Breitner, J. C., Munger, R., \& Lyketsos, C. G. (2007). Vascular factors predict rate of progression in Alzheimer disease. Neurology, 69(19), 1850-1858.

Miller, T. P., Taylor, J., Rogerson, S., Mauricio, M., Kennedy, Q., Schatzberg, A., Tinklenberg, J., \& Yesavage, J. (1998). Cognitive and noncognitive symptoms in dementia patients: relationship to cortisol and dehydroepiandrosterone. International Psychogeriatrics, 10(1), $85-96$. 
Miller, V. M., Black, D. M., Brinton, E. A., Budoff, M. J., Cedars, M. I., Hodis, H. N., Lobo, R. A., Manson, J. E., Merriam, G. R., Naftolin, F., Santoro, N., Taylor, H. S., \& Harman, S. M. (2009). Using Basic Science to Design a Clinical Trial: Baseline Characteristics of Women Enrolled in the Kronos Early Estrogen Prevention Study (KEEPS). Journal of Cardiovascular Translational Research, 2(3), 228-239.

Minzenberg, M. J., Poole, J. H., Benton, C., \& Vinogradov, S. (2004). Association of anticholinergic load with impairment of complex attention and memory in schizophrenia. American Journal of Psychiatry, 161(1), 116-124.

Moghaddam, B. (2002). Stress activation of glutamate neurotransmission in the prefrontal cortex: implications for dopamine-associated psychiatric disorders. Biological Psychiatry, 51(10), $775-787$.

Moore, G. J., Bebchuk, J. M., Wilds, I. B., Chen, G., \& Manji, H. K. (2000). Lithium-induced increase in human brain grey matter. Lancet, 356(9237), 1241-1242.

Moritz, D. J., Kasl, S.V., Ostfeld, A.M. (1992). The health impact of of living with a cognitively impaired elderly spouse. Journal of Aging Health, 4, 244-267.

Muck-Seler, D., Pivac, N., Jakovljevic, M., \& Brzovic, Z. (1999). Platelet serotonin, plasma cortisol, and dexamethasone suppression test in schizophrenic patients. Biological Psychiatry, 45(11), 1433-1439. 


\section{Transdisciplinary Allostatic Load Review}

Muck-Seler, D., Pivac, N., Mustapic, M., Crncevic, Z., Jakovljevic, M., \& Sagud, M. (2004). Platelet serotonin and plasma prolactin and cortisol in healthy, depressed and schizophrenic women. Psychiatry Research, 127(3), 217-226.

Muller, D. J., Muglia, P., Fortune, T., \& Kennedy, J. L. (2004). Pharmacogenetics of antipsychotic-induced weight gain. Pharmacological Research, 49(4), 309-329.

Muller, M., Aleman, A., Grobbee, D. E., de Haan, E. H., \& van der Schouw, Y. T. (2005). Endogenous sex hormone levels and cognitive function in aging men: is there an optimal level? Neurology, 64(5), 866-871.

Muller, M., Tang, M. X., Schupf, N., Manly, J. J., Mayeux, R., \& Luchsinger, J. A. (2007). Metabolic syndrome and dementia risk in a multiethnic elderly cohort. Dementia and Geriatric Cognitive Disorders, 24(3), 185-192.

Muntner, P., Menke, A., DeSalvo, K. B., Rabito, F. A., \& Batuman, V. (2005). Continued decline in blood lead levels among adults in the United States - The National Health and Nutrition Examination Surveys. Archives of Internal Medicine, 165(18), 2155-2161.

Mushak, P. (2003). Lead remediation and changes in human lead exposure: some physiological and biokinetic dimensions. The Science of the Total Environment, 303(1-2), 35-50.

Myers, G. J., Davidson, P. W., Shamlaye, C. F., Axtell, C. D., Cernichiari, E., Choisy, O., Choi, A., Cox, C., \& Clarkson, T. W. (1997). Effects of prenatal methylmercury exposure from a 
high fish diet on developmental milestones in the Seychelles Child Development Study. Neurotoxicology, 18(3), 819-829.

Nash, D., Magder, L., Lustberg, M., Sherwin, R. W., Rubin, R. J., Kaufmann, R. B., \& Silbergeld, E. K. (2003). Blood lead, blood pressure, and hypertension in perimenopausal and postmenopausal women. Journal of the American Medical Association, 289(12), 15231532.

Navalta, C. P., Polcari, A., Webster, D. M., Boghossian, A., \& Teicher, M. H. (2006). Effects of childhood sexual abuse on neuropsychological and cognitive function in college women. Journal of Neuropsychiatry and Clinical Neuroscience, 18(1), 45-53.

Navas-Acien, A., Guallar, E., Silbergeld, E. K., \& Rothenberg, S. J. (2007). Lead exposure and cardiovascular disease - A systematic review. Environmental Health Perspectives, 115(3), 472-482.

Needleman, H. L., Riess, J. A., Tobin, M. J., Biesecker, G. E., \& Greenhouse, J. B. (1996). Bone lead levels and delinquent behavior. Journal of the American Medical Association, 275(5), 363-369.

Newcomer, J. W. (2005). Second-generation (atypical) antipsychotics and metabolic effects: a comprehensive literature review. Central Nervous System Drugs, 19 Suppl 1, 1-93.

Noordsy, D. L., \& Green, A. I. (2003). Pharmacotherapy for schizophrenia and co-occurring substance use disorders. Current Psychiatry Reports, 5(5), 340-346. 
Notkola, I. L., Sulkava, R., Pekkanen, J., Erkinjuntti, T., Ehnholm, C., Kivinen, P., Tuomilehto, J., \& Nissinen, A. (1998). Serum total cholesterol, apolipoprotein E epsilon 4 allele, and Alzheimer's disease. Neuroepidemiology, 17(1), 14-20.

O'Brien, J. T., Lloyd, A., McKeith, I., Gholkar, A., \& Ferrier, N. (2004). A longitudinal study of hippocampal volume, cortisol levels, and cognition in older depressed subjects. American Journal of Psychiatry, 161(11), 2081-2090.

O'Brien, S. M., Scully, P., Scott, L. V., \& Dinan, T. G. (2006). Cytokine profiles in bipolar affective disorder: focus on acutely ill patients. Journal of Affective Disorders, 90(2-3), 263-267.

O'Brien, T., \& Nguyen, T. T. (1997). Lipids and lipoproteins in women. Mayo Clinical Proceedings, 72(3), 235-244.

O'Sullivan, M., Ngo, E., Viswanathan, A., Jouvent, E., Gschwendtner, A., Saemann, P. G., Duering, M., Pachai, C., Bousser, M. G., Chabriat, H., \& Dichgans, M. (2009). Hippocampal volume is an independent predictor of cognitive performance in CADASIL. [Research Support, Non-U.S. Gov't]. Neurobiology of Aging, 30(6), 890-897.

Olson, A. K., Eadie, B. D., Ernst, C., \& Christie, B. R. (2006). Environmental enrichment and voluntary exercise massively increase neurogenesis in the adult hippocampus via dissociable pathways. Hippocampus, 16(3), 250-260. 
Ortiz-Dominguez, A., Hernandez, M. E., Berlanga, C., Gutierrez-Mora, D., Moreno, J., Heinze, G., \& Pavon, L. (2007). Immune variations in bipolar disorder: phasic differences. Bipolar Disorder, 9(6), 596-602.

Ory, M. G., Hoffman, R. R., 3rd, Yee, J. L., Tennstedt, S., \& Schulz, R. (1999). Prevalence and impact of caregiving: a detailed comparison between dementia and nondementia caregivers. Gerontologist, 39(2), 177-185.

Oskam, I. C., Ropstad, E., Lie, E., Derocher, A. E., Wiig, O., Dahl, E., Larsen, S., \& Skaare, J. U. (2004). Organochlorines affect the steroid hormone cortisol in free-ranging polar bears (Ursus maritimus) at Svalbard, Norway. Journal of Toxicology and Environmental HealthPart A-Current Issues, 67(12), 959-977.

Oswald, L. M., Wong, D. F., McCaul, M., Zhou, Y., Kuwabara, H., Choi, L., Brasic, J., \& Wand, G. S. (2005). Relationships among ventral striatal dopamine release, cortisol secretion, and subjective responses to amphetamine. Neuropsychopharmacology, 30(4), 821-832.

Otte, C., Hart, S., Neylan, T. C., Marmar, C. R., Yaffe, K., \& Mohr, D. C. (2005). A meta-analysis of cortisol response to challenge in human aging: importance of gender. Psychoneuroendocrinology, 30(1), 80-91.

Ouellet-Morin, I., Boivin, M., Dionne, G., Lupien, S. J., Arseneault, L., Barr, R. G., Perusse, D., \& Tremblay, R. E. (2008). Variations in heritability of cortisol reactivity to stress as a function of early familial adversity among 19-month-old twins. Archives of General Psychiatry, 65(2), 211-218. 


\section{Transdisciplinary Allostatic Load Review}

Ownby, R. L., Crocco, E., Acevedo, A., John, V., \& Loewenstein, D. (2006). Depression and risk for Alzheimer disease: systematic review, meta-analysis, and metaregression analysis. Archives of General Psychiatry, 63(5), 530-538.

Paganini-Hill, A., \& Henderson, V. W. (1994). Estrogen deficiency and risk of Alzheimer's disease in women. American Journal of Epidemiology, 140(3), 256-261.

Patacchioli, F. R., Simeoni, S., Monnazzi, P., Pace, M., Capri, O., \& Perrone, G. (2006). Menopause, mild psychological stress and salivary cortisol: influence of long-term hormone replacement therapy (HRT). Maturitas, 55(2), 150-155.

Peavy, G. M., Salmon, D. P., Jacobson, M. W., Hervey, A., Gamst, A. C., Wolfson, T., Patterson, T. L., Goldman, S., Mills, P. J., Khandrika, S., \& Galasko, D. (2009). Effects of chronic stress on memory decline in cognitively normal and mildly impaired older adults. American Journal of Psychiatry, 166(12), 1384-1391.

Peretti, C. S., Danion, J. M., Kauffmann-Muller, F., Grange, D., Patat, A., \& Rosenzweig, P. (1997). Effects of haloperidol and amisulpride on motor and cognitive skill learning in healthy volunteers. Psychopharmacology (Berl), 131(4), 329-338.

Peters, J. L., Kubzansky, L., McNeely, E., Schwartz, J., Spiro, A., 3rd, Sparrow, D., Wright, R. O., Nie, H., \& Hu, H. (2007). Stress as a potential modifier of the impact of lead levels on blood pressure: the normative aging study. Environmental Health Perspectives, 115(8), 1154-1159. 
Petersen, R. C., Smith, G. E., Waring, S. C., Ivnik, R. J., Tangalos, E. G., \& Kokmen, E. (1999). Mild cognitive impairment: clinical characterization and outcome. Archives of Neurology, $56(3), 303-308$.

Pfeilschifter, J., Koditz, R., Pfohl, M., \& Schatz, H. (2002). Changes in proinflammatory cytokine activity after menopause. Endocrine Reviews, 23(1), 90-119.

Picard, M., Sabiston, C. M., \& McNamara, J. K. (2011). The need for a transdisciplinary, global health framework. Journal of Alternative and Complementary Medicine, 17(2), 179-184.

Pigott, T. A. (2003). Anxiety disorders in women. Psychiatric Clinics of North America, 26(3), 621-672, vi-vii.

Pirkle, J. L., Brody, D. J., Gunter, E. W., Kramer, R. A., Paschal, D. C., Flegal, K. M., \& Matte, T. D. (1994). The Decline in Blood Lead Levels in the United-States - the National-Health and Nutrition Examination Surveys (Nhanes). Journal of the American Medical Association, 272(4), 284-291.

Pirkle, J. L., Kaufmann, R. B., Brody, D. J., Hickman, T., Gunter, E. W., \& Paschal, D. C. (1998). Exposure of the U.S. population to lead, 1991-1994. Environmental Health Perspectives, 106(11), 745-750.

Plotnikoff, R. (1997). Women, heart disease and stroke in Canada: Issues and options. Ottawa: Heart and Stroke Foundation of Canada. 
Plusquellec, P., Muckle, G., Dewailly, E., Ayotte, P., Begin, G., Desrosiers, C., Despres, C., SaintAmour, D., \& Poitras, K. (2010). The relation of environmental contaminants exposure to behavioral indicators in Inuit preschoolers in Arctic Quebec. Neurotoxicology, 31(1), 1725.

Plusquellec, P., Muckle, G., Dewailly, E., Ayotte, P., Jacobson, S. W., \& Jacobson, J. L. (2007). The relation of low-level prenatal lead exposure to behavioral indicators of attention in Inuit infants in Arctic Quebec. Neurotoxicology and Teratology, 29(5), 527-537.

Ponholzer, A., Madersbacher, S., Rauchenwald, M., Jungwirth, S., Fischer, P., \& Tragl, K. H. (2009). Serum androgen levels and their association to depression and Alzheimer dementia in a cohort of 75-year-old men over 5 years: results of the VITA study. International Journal of Impotence Research, 21(3), 187-191.

Porter, R. J., \& Gallagher, P. (2006). Abnormalities of the HPA axis in affective disorders: clinical subtypes and potential treatments. Acta Neuropsychiatrica, 18(5), 193-209.

Posner, H. B., Tang, M. X., Luchsinger, J., Lantigua, R., Stern, Y., \& Mayeux, R. (2002). The relationship of hypertension in the elderly to $\mathrm{AD}$, vascular dementia, and cognitive function. Neurology, 58(8), 1175-1181.

Potkin, S. G., Fleming, K., Jin, Y., \& Gulasekaram, B. (2001). Clozapine enhances neurocognition and clinical symptomatology more than standard neuroleptics. Journal of Clinical Psychopharmacology, 21(5), 479-483. 
Potvin, S., Stip, E., Sepehry, A. A., Gendron, A., Bah, R., \& Kouassi, E. (2008). Inflammatory cytokine alterations in schizophrenia: a systematic quantitative review. Biological Psychiatry, 63(8), 801-808.

Prinz, P., Bailey, S., Moe, K., Wilkinson, C., \& Scanlan, J. (2001). Urinary free cortisol and sleep under baseline and stressed conditions in healthy senior women: effects of estrogen replacement therapy. Journal of Sleep Research, 10(1), 19-26.

Pruessner, J. C., Champagne, F., Meaney, M. J., \& Dagher, A. (2004). Dopamine release in response to a psychological stress in humans and its relationship to early life maternal care: a positron emission tomography study using [11C]raclopride. Journal of Neuroscience, $24(11), 2825-2831$.

Pugh, P. J., English, K. M., Jones, T. H., \& Channer, K. S. (2000). Testosterone: a natural tonic for the failing heart? Quarterly Journal of Medicine, 93(10), 689-694.

Rabinowitz, M. B. (1991). Toxicokinetics of bone lead. Environmental Health Perspectives, 91, $33-37$.

Raffaitin, C., Gin, H., Empana, J. P., Helmer, C., Berr, C., Tzourio, C., Portet, F., Dartigues, J. F., Alperovitch, A., \& Barberger-Gateau, P. (2009). Metabolic syndrome and risk for incident Alzheimer's disease or vascular dementia: the Three-City Study. Diabetes Care, 32(1), 169-174. 
Rahman, I., Bennet, A. M., Pedersen, N. L., de Faire, U., Svensson, P., \& Magnusson, P. K. (2009). Genetic dominance influences blood biomarker levels in a sample of 12,000 Swedish elderly twins. Twin Research and Humun Genetics, 12(3), 286-294.

Raikkonen, K., Keltikangas-Jarvinen, L., Adlercreutz, H., \& Hautanen, A. (1996). Psychosocial stress and the insulin resistance syndrome. Metabolism, 45(12), 1533-1538.

Rebuffe-Scrive, M., Marin, P., \& Bjorntorp, P. (1991). Effect of testosterone on abdominal adipose tissue in men. International Journal of Obesity, 15(11), 791-795.

Repetti, R. L., Taylor, S. E., \& Seeman, T. E. (2002). Risky families: family social environments and the mental and physical health of offspring. Psychological Bulletin, 128(2), 330-366.

Resnick, S. M., Goldszal, A. F., Davatzikos, C., Golski, S., Kraut, M. A., Metter, E. J., Bryan, R. N., \& Zonderman, A. B. (2000). One-year age changes in MRI brain volumes in older adults. Cerebral Cortex, 10(5), 464-472.

Reul, J. M., \& de Kloet, E. R. (1985). Two receptor systems for corticosterone in rat brain: microdistribution and differential occupation. Endocrinology, 117(6), 2505-2511.

Risch, N., Herrell, R., Lehner, T., Liang, K. Y., Eaves, L., Hoh, J., Griem, A., Kovacs, M., Ott, J., \& Merikangas, K. R. (2009). Interaction between the serotonin transporter gene (5HTTLPR), stressful life events, and risk of depression: a meta-analysis. Journal of the American Medical Association, 301(23), 2462-2471. 
Ritsner, M., Gibel, A., Maayan, R., Ratner, Y., Ram, E., Biadsy, H., Modai, I., \& Weizman, A. (2005). Cortisol/dehydroepiandrosterone ratio and responses to antipsychotic treatment in schizophrenia. Neuropsychopharmacology, 30(10), 1913-1922.

Ritsner, M., Gibel, A., Maayan, R., Ratner, Y., Ram, E., Modai, I., \& Weizman, A. (2007). State and trait related predictors of serum cortisol to $\operatorname{DHEA}(\mathrm{S})$ molar ratios and hormone concentrations in schizophrenia patients. European Journal of Neuropsychopharmacology, $17(4), 257-264$.

Rivier, C., \& Rivest, S. (1991). Effect of stress on the activity of the hypothalamic-pituitarygonadal axis: peripheral and central mechanisms. Biology of Reproduction, 45(4), 523-532.

Roca, C. A., Schmidt, P. J., Deuster, P. A., Danaceau, M. A., Altemus, M., Putnam, K., Chrousos, G. P., Nieman, L. K., \& Rubinow, D. R. (2005). Sex-related differences in stimulated hypothalamic-pituitary-adrenal axis during induced gonadal suppression. Journal of Clinical Endocrinology and Metabolism, 90(7), 4224-4231.

Rocca, W. A., Bower, J. H., Maraganore, D. M., Ahlskog, J. E., Grossardt, B. R., de Andrade, M., \& Melton, L. J., 3rd. (2007). Increased risk of cognitive impairment or dementia in women who underwent oophorectomy before menopause. Neurology, 69(11), 1074-1083.

Rosenfield, P. L. (1992). The potential of transdisciplinary research for sustaining and extending linkages between the health and social sciences. Social Science and Medicine, 35(11), $1343-1357$. 


\section{Transdisciplinary Allostatic Load Review}

Ross, L. E., \& McLean, L. M. (2006). Anxiety disorders during pregnancy and the postpartum period: A systematic review. Journal of Clinical Psychiatry, 67(8), 1285-1298.

Rossouw, J. E., Cushman, M., Greenland, P., Lloyd-Jones, D. M., Bray, P., Kooperberg, C., Pettinger, M., Robinson, J., Hendrix, S., \& Hsia, J. (2008). Inflammatory, lipid, thrombotic, and genetic markers of coronary heart disease risk in the women's health initiative trials of hormone therapy. Archives of Internal Medicine, 168(20), 2245-2253.

Rossouw, J. E., Prentice, R. L., Manson, J. E., Wu, L., Barad, D., Barnabei, V. M., Ko, M., LaCroix, A. Z., Margolis, K. L., \& Stefanick, M. L. (2007). Postmenopausal hormone therapy and risk of cardiovascular disease by age and years since menopause. Journal of the American Medical Association, 297(13), 1465-1477.

Roth, T. L., Lubin, F. D., Sodhi, M., \& Kleinman, J. E. (2009). Epigenetic mechanisms in schizophrenia. Biochimica et Biophysica Acta, 1790(9), 869-877.

Rutter, M., \& Sroufe, L. A. (2000). Developmental psychopathology: concepts and challenges. Development and Psychopathology, 12(3), 265-296.

Ryan, M. C., Sharifi, N., Condren, R., \& Thakore, J. H. (2004). Evidence of basal pituitaryadrenal overactivity in first episode, drug naive patients with schizophrenia. Psychoneuroendocrinology, 29(8), 1065-1070. 
Saari, K., Koponen, H., Laitinen, J., Jokelainen, J., Lauren, L., Isohanni, M., \& Lindeman, S. (2004). Hyperlipidemia in persons using antipsychotic medication: a general populationbased birth cohort study. Journal of Clinical Psychiatry, 65(4), 547-550.

Saeedi, H., Remington, G., \& Christensen, B. K. (2006). Impact of haloperidol, a dopamine D2 antagonist, on cognition and mood. Schizophrenia Research, 85(1-3), 222-231.

Salonen, J. T., Seppanen, K., Nyyssonen, K., Korpela, H., Kauhanen, J., Kantola, M., Tuomilehto, J., Esterbauer, H., Tatzber, F., \& Salonen, R. (1995). Intake of Mercury from Fish, LipidPeroxidation, and the Risk of Myocardial-Infarction and Coronary, Cardiovascular, and Any Death in Eastern Finnish Men. Circulation, 91(3), 645-655.

Sameroff, A. (2009). The transactional model of development: how children and contexts shape each other: American Psychological Association.

Sameroff, A. J. (2000). Developmental systems and psychopathology. Development and Psychopathology, 12(3), 297-312.

Sameroff, A. J., \& Mackenzie, M. J. (2003). Research strategies for capturing transactional models of development: the limits of the possible. Development and Psychopathology, $15(3), 613-640$.

Sanchez, M. M., Young, L. J., Plotsky, P. M., \& Insel, T. R. (2000). Distribution of corticosteroid receptors in the rhesus brain: relative absence of glucocorticoid receptors in the hippocampal formation. Journal of Neuroscience, 20(12), 4657-4668. 
Sapolsky, R. M. (2004). Organismal stress and telomeric aging: an unexpected connection. Proceedings of the National Academy of Sciences of the United States of America, 101(50), 17323-17324.

Sapolsky, R. M. (2005). The influence of social hierarchy on primate health. Science, 308(5722), 648-652.

Sapolsky, R. M., Krey, L. C., \& McEwen, B. S. (1986). The neuroendocrinology of stress and aging: the glucocorticoid cascade hypothesis. Endocrine Reviews, 7(3), 284-301.

Sapolsky, R. M., Romero, L. M., \& Munck, A. U. (2000). How do glucocorticoids influence stress responses? Integrating permissive, suppressive, stimulatory, and preparative actions. Endocrine Reviews, 21(1), 55-89.

Schleuning, M. J., Duggan, A., \& Reem, G. H. (1989). Inhibition by chlorpromazine of lymphokine-specific mRNA expression in human thymocytes. European Journal of Immunology, 19(8), 1491-1495.

Schmidt, R., Schmidt, H., Curb, J. D., Masaki, K., White, L. R., \& Launer, L. J. (2002). Early inflammation and dementia: a 25-year follow-up of the Honolulu-Asia Aging Study. Annals of Neurology, 52(2), 168-174.

Schnaider Beeri, M., Goldbourt, U., Silverman, J. M., Noy, S., Schmeidler, J., Ravona-Springer, R., Sverdlick, A., \& Davidson, M. (2004). Diabetes mellitus in midlife and the risk of 
dementia three decades later. [Research Support, Non-U.S. Gov't]. Neurology, 63(10), 1902-1907.

Schnorpfeil, P., Noll, A., Schulze, R., Ehlert, U., Frey, K., \& Fischer, J. E. (2003). Allostatic load and work conditions. Social Science and Medicine, 57(4), 647-656.

Schram, M. T., Euser, S. M., de Craen, A. J., Witteman, J. C., Frolich, M., Hofman, A., Jolles, J., Breteler, M. M., \& Westendorp, R. G. (2007). Systemic markers of inflammation and cognitive decline in old age. Journal of the American Geriatric Society, 55(5), 708-716.

Schuepbach, D., Keshavan, M. S., Kmiec, J. A., \& Sweeney, J. A. (2002). Negative symptom resolution and improvements in specific cognitive deficits after acute treatment in firstepisode schizophrenia. Schizophrenia Research, 53(3), 249-261.

Schule, C. (2007). Neuroendocrinological mechanisms of actions of antidepressant drugs. Journal of Neuroendocrinology, 19(3), 213-226.

Schulkin, J. (2003a). Addiction to drugs: allostatic regulation under duress Rethinking Homestasis: Allostatic regulation in physiology and pathophysiology (pp. 131-160). Cambridge, MA: MIT Press.

Schulkin, J. (2003b). Allostasis: a neural behavioral perspective. Hormones and Behavior, 43(1), 21-27; discussion 28-30. 
Schulkin, J., Gold, P. W., \& McEwen, B. S. (1998). Induction of corticotropin-releasing hormone gene expression by glucocorticoids: implication for understanding the states of fear and anxiety and allostatic load. Psychoneuroendocrinology, 23(3), 219-243.

Schulkin, J., McEwen, B. S., \& Gold, P. W. (1994). Allostasis, amygdala, and anticipatory angst. Neuroscience and Biobehavioral Reviews, 18(3), 385-396.

Schulz, R., \& Beach, S. R. (1999). Caregiving as a risk factor for mortality: the Caregiver Health Effects Study. Journal of the American Medical Association, 282(23), 2215-2219.

Schulz, R., Hebert, R. S., Dew, M. A., Brown, S. L., Scheier, M. F., Beach, S. R., Czaja, S. J., Martire, L. M., Coon, D., Langa, K. M., Gitlin, L. N., Stevens, A. B., \& Nichols, L. (2007). Patient suffering and caregiver compassion: new opportunities for research, practice, and policy. Gerontologist, 47(1), 4-13.

Schulz, R., McGinnis, K. A., Zhang, S., Martire, L. M., Hebert, R. S., Beach, S. R., Zdaniuk, B., Czaja, S. J., \& Belle, S. H. (2008). Dementia patient suffering and caregiver depression. Alzheimer's Disease and Associated Disorders, 22(2), 170-176.

Schulz, R., Newsom, J., Mittelmark, M., Burton, L., Hirsch, C., \& Jackson, S. (1997). Health effects of caregiving: the caregiver health effects study: an ancillary study of the Cardiovascular Health Study. Annals of Behavioral Medicine, 19(2), 110-116. 
Schulz, R., O'Brien, A. T., Bookwala, J., \& Fleissner, K. (1995). Psychiatric and physical morbidity effects of dementia caregiving: prevalence, correlates, and causes. Gerontologist, 35(6), 771-791.

Schulz, R., Visintainer, P., \& Williamson, G. M. (1990). Psychiatric and physical morbidity effects of caregiving. Journal of Gerontology, 45(5), P181-191.

Seeman, E., Singer, B. H., Rowe, J., Horwitz, R. I., \& McEwen, B. (1997a). Price of adaptation allostatic load and its health consequences. Archives of Internal Medicine, 157, 2259-2268.

Seeman, T., Glei, D., Goldman, N., Weinstein, M., Singer, B., \& Lin, Y. H. (2004). Social relationships and allostatic load in Taiwanese elderly and near elderly. Social Science and Medicine, 59(11), 2245-2257.

Seeman, T., Gruenewald, T., Karlamangla, A., Sidney, S., Liu, K., McEwen, B., \& Schwartz, J. (2009). Modeling multisystem biological risk in young adults: The Coronary Artery Risk Development in Young Adults Study. American Journal of Human Biology, 22(4), 463472.

Seeman, T. E., Dubin, L. F., \& Seeman, M. (2003). Religiosity/spirituality and health. A critical review of the evidence for biological pathways. American Psychologist, 58(1), 53-63.

Seeman, T. E., McEwen, B. S., Rowe, J. W., \& Singer, B. H. (2001). Allostatic load as a marker of cumulative biological risk: MacArthur studies of successful aging. Proceedings of the National Academy of the United States of America, 98(8), 4770-4775. 
Seeman, T. E., McEwen, B. S., Singer, B. H., Albert, M. S., \& Rowe, J. W. (1997b). Increase in urinary cortisol excretion and memory declines: MacArthur studies of successful aging. Journal of Clinical Endocrinology and Metabolism, 82(8), 2458-2465.

Seeman, T. E., Singer, B. H., Ryff, C. D., Dienberg Love, G., \& Levy-Storms, L. (2002). Social relationships, gender, and allostatic load across two age cohorts. Psychosomatic Medicine, 64(3), 395-406.

Seplaki, C. L., Goldman, N., Glei, D., \& Weinstein, M. (2005). A comparative analysis of measurement approaches for physiological dysregulation in an older population. Experimental Gerontology, 40(5), 438-449.

Seplaki, C. L., Goldman, N., Weinstein, M., \& Lin, Y. H. (2004). How are biomarkers related to physical and mental well-being? Journal of Gerentology, 59A(3), 201-217.

Seplaki, C. L., Goldman, N., Weinstein, M., \& Lin, Y. H. (2006). Measurement of cumulative physiological dysregulation in an older population. Demography, 43(1), 165-183.

Sexton, K. (1997). Sociodemographic aspects of human susceptibility to toxic chemicals: Do class and race matter for realistic risk assessment? [Proceedings Paper]. Environmental Toxicology and Pharmacology, 4(3-4), 261-269.

Sexton, K., Adgate, J. L., Fredrickson, A. L., Ryan, A. D., Needham, L. L., \& Ashley, D. L. (2006). Using biologic markers in blood to assess exposure to multiple environmental 
chemicals for inner-city children 3-6 years of age. Environmental Health Perspectives, $114(3), 453-459$.

Sexton, K., Greaves, I. A., Church, T. R., Adgate, J. L., Ramachandran, G., Tweedie, R. L., Fredrickson, A., Geisser, M., Sikorski, M., Fischer, G., Jones, D., \& Ellringer, P. (2000). A school-based strategy to assess children's environmental exposures and related health effects in economically disadvantaged urban neighborhoods. Journal of Exposure Analysis and Environmental Epidemiology, 10(6), 682-694.

Shanks-McElroy, H. A., \& Strobino, J. (2001). Male caregivers of spouses with Alzheimer's disease: risk factors and health status. American Journal of Alzheimers Disease and Other Dementias, 16(3), 167-175.

Sheline, Y. I., Sanghavi, M., Mintun, M. A., \& Gado, M. H. (1999). Depression duration but not age predicts hippocampal volume loss in medically healthy women with recurrent major depression. Journal of Neuroscience, 19(12), 5034-5043.

Sher, L. (2006). Combined dexamethasone suppression-corticotropin-releasing hormone stimulation test in studies of depression, alcoholism, and suicidal behavior. Scientific World Journal, 6, 1398-1404.

Sherwin, B. B. (2007). The critical period hypothesis: can it explain discrepancies in the oestrogen-cognition literature? Journal of Neuroendocrinology, 19(2), 77-81. 
Shimizu, E., Hashimoto, K., Okamura, N., Koike, K., Komatsu, N., Kumakiri, C., Nakazato, M., Watanabe, H., Shinoda, N., Okada, S., \& Iyo, M. (2003). Alterations of serum levels of brain-derived neurotrophic factor (BDNF) in depressed patients with or without antidepressants. Biological Psychiatry, 54(1), 70-75.

Shonkoff, J. P., Boyce, W. T., \& McEwen, B. S. (2009). Neuroscience, molecular biology, and the childhood roots of health disparities: building a new framework for health promotion and disease prevention. Journal of the American Medical Association, 301(21), 2252-2259.

Simerly, R. B., Chang, C., Muramatsu, M., \& Swanson, L. W. (1990). Distribution of androgen and estrogen receptor mRNA-containing cells in the rat brain: an in situ hybridization study. The Journal of Comparative Neurology, 294(1), 76-95.

Singer, B., Friedman, E., Seeman, T., Fava, G. A., \& Ryff, C. D. (2005). Protective environments and health status: cross-talk between human and animal studies. Neurobiology of Aging, 26 Suppl 1, 113-118.

Singer, B., \& Ryff, C. D. (1999). Hierarchies of life histories and associated health risks. Annals of the New York Academy of Science, 896, 96-115.

Skoog, I., Lernfelt, B., Landahl, S., Palmertz, B., Andreasson, L. A., Nilsson, L., Persson, G., Oden, A., \& Svanborg, A. (1996). 15-year longitudinal study of blood pressure and dementia. Lancet, 347(9009), 1141-1145. 
Smith, A. K., Maloney, E. M., Falkenberg, V. R., Dimulescu, I., \& Rajeevan, M. S. (2009). An angiotensin-1 converting enzyme polymorphism is associated with allostatic load mediated by C-reactive protein, interleukin-6 and cortisol. Psychoneuroendocrinology, 34(4), 597606.

Smith, M. E. (2005). Bilateral hippocampal volume reduction in adults with post-traumatic stress disorder: a meta-analysis of structural MRI studies. Hippocampus, 15(6), 798-807.

Smolka, M. N., Schumann, G., Wrase, J., Grusser, S. M., Flor, H., Mann, K., Braus, D. F., Goldman, D., Buchel, C., \& Heinz, A. (2005). Catechol-O-methyltransferase val158met genotype affects processing of emotional stimuli in the amygdala and prefrontal cortex. Journal of Neuroscience, 25(4), 836-842.

Solfrizzi, V., Scafato, E., Capurso, C., D'Introno, A., Colacicco, A. M., Frisardi, V., Vendemiale, G., Baldereschi, M., Crepaldi, G., Di Carlo, A., Galluzzo, L., Gandin, C., Inzitari, D., Maggi, S., Capurso, A., \& Panza, F. (2009). Metabolic syndrome and the risk of vascular dementia: the Italian Longitudinal Study on Ageing. Journal of Neurology, Neurosurgery, and Psychiatry, 81(4), 433-440.

Solfrizzi, V., Scafato, E., Capurso, C., D'Introno, A., Colacicco, A. M., Frisardi, V., Vendemiale, G., Baldereschi, M., Crepaldi, G., Di Carlo, A., Galluzzo, L., Gandin, C., Inzitari, D., Maggi, S., Capurso, A., \& Panza, F. (In Press). Metabolic syndrome, mild cognitive impairment, and progression to dementia. The Italian Longitudinal Study on Aging. Neurobiology of Aging. 
Songdej, N., Winters, P. C., McCabe, M. J., Jr., \& van Wijngaarden, E. (2010). A populationbased assessment of blood lead levels in relation to inflammation. Environmental Research, 110(3), 272-277.

Spiegel, K., Tasali, E., Penev, P., \& Van Cauter, E. (2004). Brief communication: Sleep curtailment in healthy young men is associated with decreased leptin levels, elevated ghrelin levels, and increased hunger and appetite. Annals of Internal Medicine, 141(11), 846-850.

Steffens, D. C., Byrum, C. E., McQuoid, D. R., Greenberg, D. L., Payne, M. E., Blitchington, T. F., MacFall, J. R., \& Krishnan, K. R. (2000). Hippocampal volume in geriatric depression. Biological Psychiatry, 48(4), 301-309.

Stein, J., Schettler, T., Wallinga, D., \& Valenti, M. (2002). In harm's way: toxic threats to child development. Journal of Developmental and Behavioral Pediatrics, 23(1 Suppl), S13-22.

Steiner, M., Dunn, E., \& Born, L. (2003). Hormones and mood: from menarche to menopause and beyond. Journal of Affective Disorders, 74(1), 67-83.

Stephens, J. W., Dhamrait, S. S., Cooper, J. A., Acharya, J., Miller, G. J., Hurel, S. J., \& Humphries, S. E. (2005). The D allele of the ACE I/D common gene variant is associated with Type 2 diabetes mellitus in Caucasian subjects. Molecular Genetics and Metabolism, 84(1), 83-89. 
Steptoe, A., van Jaarsveld, C. H., Semmler, C., Plomin, R., \& Wardle, J. (2009). Heritability of daytime cortisol levels and cortisol reactivity in children. Psychoneuroendocrinology, $34(2), 273-280$.

Sterling, P. (2004). Principles of allostasis: optimal design, predictive regulation, pathophysiology and rational therapeutics. In J. Schulkin (Ed.), Allostasis, homeostasis, and the costs of adaptation (pp. 17). Cambridge: Cambridge Unversity Press.

Sterling, P., \& Eyer, J. (1988). Allostasis: a new paradigm to explain arousal pathology. In S. Fisher \& J. Reason (Eds.), Handbook of Life Stress, Cognition and Health (pp. 629-649). New York: John Wiley \& Sons.

Stern, A. H. (2005). A review of the studies of the cardiovascular health effects of methylmercury with consideration of their suitability for risk assessment. Environmental Research, 98(1), 133-142.

Stewart, R., Masaki, K., Xue, Q. L., Peila, R., Petrovitch, H., White, L. R., \& Launer, L. J. (2005). A 32-year prospective study of change in body weight and incident dementia: the Honolulu-Asia Aging Study. Archives of Neurology, 62(1), 55-60.

Stewart, R., White, L. R., Xue, Q. L., \& Launer, L. J. (2007). Twenty-six-year change in total cholesterol levels and incident dementia: the Honolulu-Asia Aging Study. Archives of Neurology, 64(1), 103-107. 


\section{Transdisciplinary Allostatic Load Review}

Stip, E., Remington, G. J., Dursun, S. M., Reiss, J. P., Rotstein, E., MacEwan, G. W., Chokka, P. R., Jones, B., \& Dickson, R. A. (2003). A Canadian multicenter trial assessing memory and executive functions in patients with schizophrenia spectrum disorders treated with olanzapine. Journal of Clinical Psychopharmacology, 23(4), 400-404.

Stroud, L. R., Salovey, P., \& Epel, E. S. (2002). Sex differences in stress responses: social rejection versus achievement stress. Biological Psychiatry, 52(4), 318-327.

Sullivan, E. V., Pfefferbaum, A., Swan, G. E., \& Carmelli, D. (2001). Heritability of hippocampal size in elderly twin men: equivalent influence from genes and environment. Hippocampus, 11(6), 754-762.

Sun, J., Wang, S., Zhang, J. Q., \& Li, W. (2007). Assessing the cumulative effects of stress: the association between job stress and allostatic load in a large sample of Chinese employees. Work and Stress, 21(4), 333-347.

Suomi, S. J., Delizio, R., \& Harlow, H. F. (1976). Social rehabilitation of separation-induced depressive disorders in monkeys. American Journal of Psychiatry, 133(11), 1279-1285.

Swann, A. C. (2001). Major system toxicities and side effects of anticonvulsants. Journal of Clinical Psychiatry, 62 Suppl 14, 16-21.

Swanwick, G. R., Kirby, M., Bruce, I., Buggy, F., Coen, R. F., Coakley, D., \& Lawlor, B. A. (1998). Hypothalamic-pituitary-adrenal axis dysfunction in Alzheimer's disease: lack of 
association between longitudinal and cross-sectional findings. American Journal of Psychiatry, 155(2), 286-289.

Szanton, S. L., Allen, J. K., Seplaki, C. L., Bandeen-Roche, K., \& Fried, L. P. (2009). Allostatic load and frailty in the women's health and aging studies. Biological Research for Nursing, $10(3), 248-256$.

Szmuilowicz, E. D., Stuenkel, C. A., \& Seely, E. W. (2009). Influence of menopause on diabetes and diabetes risk. Nature Reviews. Endocrinology, 5(10), 553-558.

Szyf, M., Weaver, I. C., Champagne, F. A., Diorio, J., \& Meaney, M. J. (2005). Maternal programming of steroid receptor expression and phenotype through DNA methylation in the rat. Frontiers in Neuroendocrinology, 26(3-4), 139-162.

Tan, Z. S., Seshadri, S., Beiser, A., Wilson, P. W., Kiel, D. P., Tocco, M., D'Agostino, R. B., \& Wolf, P. A. (2003). Plasma total cholesterol level as a risk factor for Alzheimer disease: the Framingham Study. Archives of Internal Medicine, 163(9), 1053-1057.

Tarkowski, E., Andreasen, N., Tarkowski, A., \& Blennow, K. (2003). Intrathecal inflammation precedes development of Alzheimer's disease. Journal of Neurology, Neurosurgery, and Psychiatry, 74(9), 1200-1205.

Tasali, E., Leproult, R., Ehrmann, D. A., \& Van Cauter, E. (2008). Slow-wave sleep and the risk of type 2 diabetes in humans. Proceedings of the National Academy of Science of the United States of America, 105(3), 1044-1049. 


\section{Transdisciplinary Allostatic Load Review}

Taylor, S. E., Klein, L. C., Lewis, B. P., Gruenewald, T. L., Gurung, R. A., \& Updegraff, J. A. (2000). Biobehavioral responses to stress in females: tend-and-befriend, not fight-or-flight. Psychological Reviews, 107(3), 411-429.

Taylor, S. E., Repetti, R. L., \& Seeman, T. (1997). Health psychology: what is an unhealthy environment and how does it get under the skin? Annual Review of Psychology, 48, 411447.

Teicher, M. H., Andersen, S. L., Polcari, A., Anderson, C. M., Navalta, C. P., \& Kim, D. M. (2003). The neurobiological consequences of early stress and childhood maltreatment. Neuroscience and Biobehavioral Reviews, 27(1-2), 33-44.

Terburg, D., Morgan, B., \& van Honk, J. (2009). The testosterone-cortisol ratio: A hormonal marker for proneness to social aggression. International Journal of Law and Psychiatry, 32(4), 216-223.

Thayer, J. F., \& Lane, R. D. (2009). Claude Bernard and the heart-brain connection: further elaboration of a model of neurovisceral integration. Neuroscience and Biobehavioral Reviews, 33(2), 81-88.

Tilbrook, A. J., Turner, A. I., \& Clarke, I. J. (2000). Effects of stress on reproduction in nonrodent mammals: the role of glucocorticoids and sex differences. Reviews of Reproduction, $5(2), 105-113$. 
Tkacova, R., Joppa, P., Stancak, B., Salagovic, J., Misikova, S., \& Kalina, I. (2005). The link between angiotensin-converting enzyme genotype and pulmonary artery pressure in patients with COPD. Wiener Klinische Wochenschrift, 117(5-6), 210-214.

Traish, A. M., Guay, A., Feeley, R., \& Saad, F. (2009a). The dark side of testosterone deficiency: I. Metabolic syndrome and erectile dysfunction. Journal of Andrology, 30(1), 10-22.

Traish, A. M., Saad, F., Feeley, R. J., \& Guay, A. (2009b). The dark side of testosterone deficiency: III. Cardiovascular disease. Journal of Andrology, 30(5), 477-494.

Traish, A. M., Saad, F., \& Guay, A. (2009c). The dark side of testosterone deficiency: II. Type 2 diabetes and insulin resistance. Journal of Andrology, 30(1), 23-32.

Trayhurn, P., \& Wood, I. S. (2004). Adipokines: inflammation and the pleiotropic role of white adipose tissue. British Journal of Nutrition, 92(3), 347-355.

Tsai, S. Y., Chen, K. P., Yang, Y. Y., Chen, C. C., Lee, J. C., Singh, V. K., \& Leu, S. J. (1999). Activation of indices of cell-mediated immunity in bipolar mania. Biological Psychiatry, 45(8), 989-994.

Tsukasaki, K., Kido, T., Makimoto, K., Naganuma, R., Ohno, M., \& Sunaga, K. (2006). The impact of sleep interruptions on vital measurements and chronic fatigue of female caregivers providing home care in Japan. Nursing and Health Sciences, 8(1), 2-9. 
Uemura, H., Arisawa, K., Hiyoshi, M., Kitayama, A., Takami, H., Sawachika, F., Dakeshita, S., Nii, K., Satoh, H., Sumiyoshi, Y., Morinaga, K., Kodama, K., Suzuki, T., \& Nagai, M. (2009). Prevalence of metabolic syndrome associated with body burden levels of dioxin and related compounds among Japan's general population. Environmental Health Perspectives, 117(4), 568-573.

Uhart, M., Chong, R. Y., Oswald, L., Lin, P. I., \& Wand, G. S. (2006). Gender differences in hypothalamic-pituitary-adrenal (HPA) axis reactivity. Psychoneuroendocrinology, 31(5), 642-652.

Umegaki, H., Ikari, H., Nakahata, H., Endo, H., Suzuki, Y., Ogawa, O., Nakamura, A., Yamamoto, T., \& Iguchi, A. (2000). Plasma cortisol levels in elderly female subjects with Alzheimer's disease: a cross-sectional and longitudinal study. Brain Research, 881(2), 241243.

Valdez, G. R., \& Koob, G. F. (2004). Allostasis and dysregulation of corticotropin-releasing factor and neuropeptide $\mathrm{Y}$ systems: implications for the development of alcoholism. Pharmacology, Biochemistry, and Behavior, 79(4), 671-689.

Van Cauter, E., Holmback, U., Knutson, K., Leproult, R., Miller, A., Nedeltcheva, A., Pannain, S., Penev, P., Tasali, E., \& Spiegel, K. (2007). Impact of sleep and sleep loss on neuroendocrine and metabolic function. Hormone Research, 67 Suppl 1, 2-9. 
van den Berg, E., Biessels, G. J., de Craen, A. J., Gussekloo, J., \& Westendorp, R. G. (2007). The metabolic syndrome is associated with decelerated cognitive decline in the oldest old. Neurology, 69(10), 979-985.

van Haren, N. E., Hulshoff Pol, H. E., Schnack, H. G., Cahn, W., Mandl, R. C., Collins, D. L., Evans, A. C., \& Kahn, R. S. (2007). Focal gray matter changes in schizophrenia across the course of the illness: a 5-year follow-up study. Neuropsychopharmacology, 32(10), 20572066.

van Oijen, M., Witteman, J. C., Hofman, A., Koudstaal, P. J., \& Breteler, M. M. (2005). Fibrinogen is associated with an increased risk of Alzheimer disease and vascular dementia. Stroke, 36(12), 2637-2641.

Vendsborg, P. B., Bech, P., \& Rafaelsen, O. J. (1976). Lithium treatment and weight gain. Acta Psychiatrica Scandaviva, 53(2), 139-147.

Vermetten, E., Vythilingam, M., Southwick, S. M., Charney, D. S., \& Bremner, J. D. (2003). Long-term treatment with paroxetine increases verbal declarative memory and hippocampal volume in posttraumatic stress disorder. Biological Psychiatry, 54(7), 693702.

Viau, V. (2002). Functional cross-talk between the hypothalamic-pituitary-gonadal and -adrenal axes. Journal of Neuroendocrinology, 14(6), 506-513. 


\section{Transdisciplinary Allostatic Load Review}

Videbech, P., \& Ravnkilde, B. (2004). Hippocampal volume and depression: A meta-analysis of MRI studies. American Journal of Psychiatry, 161(11), 1957-1966.

Vieta, E., Gasto, C., Martinez de Osaba, M. J., Nieto, E., Canto, T. J., Otero, A., \& Vallejo, J. (1997). Prediction of depressive relapse in remitted bipolar patients using corticotrophinreleasing hormone challenge test. Acta Psychiatrica Scandinavica, 95(3), 205-211.

Vinberg, M., Bennike, B., Kyvik, K. O., Andersen, P. K., \& Kessing, L. V. (2008). Salivary cortisol in unaffected twins discordant for affective disorder. Psychiatry Research, 161(3), 292-301.

Vinberg, M., Mortensen, E. L., Kyvik, K. O., \& Kessing, L. V. (2007). Personality traits in unaffected twins discordant for affective disorder. Acta Psychiatrica Scandinavica, 115(6), $442-450$.

Vinberg, M., Trajkovska, V., Bennike, B., Knorr, U., Knudsen, G. M., \& Kessing, L. V. (2009). The BDNF Val66Met polymorphism: relation to familiar risk of affective disorder, BDNF levels and salivary cortisol. Psychoneuroendocrinology, 34(9), 1380-1389.

Virgolini, M. B., Chen, K., Weston, D. D., Bauter, M. R., \& Cory-Slechta, D. A. (2005). Interactions of chronic lead exposure and intermittent stress: consequences for brain catecholamine systems and associated behaviors and HPA axis function. Toxicological Sciences, 87(2), 469-482. 
Virgolini, M. B., Rossi-George, A., Weston, D., \& Cory-Slechta, D. A. (2008). Influence of low level maternal $\mathrm{Pb}$ exposure and prenatal stress on offspring stress challenge responsivity. Neurotoxicology, 29(6), 928-939.

Vitaliano, P. P., Russo, J., Bailey, S.L., Young, H.M., McCann, B.S. (1993). Psychosocial factors associated with cardiovascular reactivity in older adults. Psychosomatic Medicine, 55, 164177.

Vitaliano, P. P., Zhang, J., \& Scanlan, J. M. (2003). Is caregiving hazardous to one's physical health? A meta-analysis. Psychological Bulletin, 129(6), 946-972.

von Kanel, R., Dimsdale, J. E., Patterson, T. L., \& Grant, I. (2003). Acute procoagulant stress response as a dynamic measure of allostatic load in Alzheimer caregivers. Annals of Behavioral Medicine, 26(1), 42-48.

von Thiele, U., Lindfors, P., \& Lundberg, U. (2006). Self-rated recovery from work stress and allostatic load in women. Journal of Psychosomatic Research, 61(2), 237-242.

Voruganti, L., \& Awad, A. G. (2004). Neuroleptic dysphoria: towards a new synthesis. Psychopharmacology (Berlin), 171(2), 121-132.

Vyskocil, A., Fiala, Z., Ettlerova, E., \& Tenjnorova, I. (1990). Influence of chronic lead exposure on hormone levels in developing rats. Journal of Applied Toxicology, 10(4), 301-302. 
Vythilingam, M., Heim, C., Newport, J., Miller, A. H., Anderson, E., Bronen, R., Brummer, M., Staib, L., Vermetten, E., Charney, D. S., Nemeroff, C. B., \& Bremner, J. D. (2002). Childhood trauma associated with smaller hippocampal volume in women with major depression. American Journal of Psychiatry, 159(12), 2072-2080.

Vythilingam, M., Vermetten, E., Anderson, G. M., Luckenbaugh, D., Anderson, E. R., Snow, J., Staib, L. H., Charney, D. S., \& Bremner, J. D. (2004). Hippocampal volume, memory, and cortisol status in major depressive disorder: effects of treatment. Biological Psychiatry, $56(2), 101-112$.

Walker, E., Mittal, V., \& Tessner, K. (2008). Stress and the hypothalamic pituitary adrenal axis in the developmental course of schizophrenia. Annual Review in Clinical Psychology, 4, 189216.

Wallace, D. C. (2005). A mitochondrial paradigm of metabolic and degenerative diseases, aging, and cancer: a dawn for evolutionary medicine. Annual Review in Genetics, 39, 359-407.

Walsh, P., Spelman, L., Sharifi, N., \& Thakore, J. H. (2005). Male patients with paranoid schizophrenia have greater ACTH and cortisol secretion in response to metoclopramideinduced AVP release. Psychoneuroendocrinology, 30(5), 431-437.

Warren, M. F., Serby, M. J., \& Roane, D. M. (2008). The effects of testosterone on cognition in elderly men: a review. CNS Spectrums, 13(10), 887-897. 
Watson, S., Gallagher, P., Ritchie, J. C., Ferrier, I. N., \& Young, A. H. (2004). Hypothalamicpituitary-adrenal axis function in patients with bipolar disorder. British Journal of Psychiatry, 184, 496-502.

Way, B. M., \& Taylor, S. E. (2010). The serotonin transporter promoter polymorphism is associated with cortisol response to psychosocial stress. Biological Psychiatry, 67(5), 487492.

Weaver, I. C., Cervoni, N., Champagne, F. A., D'Alessio, A. C., Sharma, S., Seckl, J. R., Dymov, S., Szyf, M., \& Meaney, M. J. (2004). Epigenetic programming by maternal behavior. Nature Neuroscience, 7(8), 847-854.

Weaver, J. D., Huang, M. H., Albert, M., Harris, T., Rowe, J. W., \& Seeman, T. E. (2002). Interleukin-6 and risk of cognitive decline: MacArthur studies of successful aging. Neurology, 59(3), 371-378.

Weiner, M. F., Vobach, S., Olsson, K., Svetlik, D., \& Risser, R. C. (1997). Cortisol secretion and Alzheimer's disease progression. Biological Psychiatry, 42(11), 1030-1038.

Weisglas-Kuperus, N., Vreugdenhil, H. J., \& Mulder, P. G. (2004). Immunological effects of environmental exposure to polychlorinated biphenyls and dioxins in Dutch school children. Toxicology Letters, 149(1-3), 281-285.

Weiss, B. (2007). Can endocrine disruptors influence neuroplasticity in the aging brain? Neurotoxicology, 28(5), 938-950. 
Whitmer, R. A., Sidney, S., Selby, J., Johnston, S. C., \& Yaffe, K. (2005). Midlife cardiovascular risk factors and risk of dementia in late life. Neurology, 64(2), 277-281.

WHO. (1999). Occupational health, ethically correct, economically sound. World Health Organization (Fact Sheet Nr 84).

WHO. (2001). The World Health Report 2001 - Mental Health: New Understanding, New Hope, Geneva.

WHO. (2002). Active aging: a policy framework.

WHO. (2002). Global Assessment of the state-of-the-science of Endocrine Disruptors.

Wilcox, S., \& King, A. C. (1999). Sleep complaints in older women who are family caregivers. Journals of Gerontology. Series B: Psychological Science and Social Science, 54(3), P189198.

Willette-Murphy, K., Todero, C., \& Yeaworth, R. (2006). Mental health and sleep of older wife caregivers for spouses with Alzheimer's disease and related disorders. Issues in Mental Health and Nursing, 27(8), 837-852.

Williams, P. T., Blanche, P. J., \& Krauss, R. M. (2005). Behavioral versus genetic correlates of lipoproteins and adiposity in identical twins discordant for exercise. Circulation, 112(3), $350-356$. 
Wolf, O. T., \& Kudielka, B. M. (2008). Stress, health and ageing: a focus on postmenopausal women. Menopause International, 14(3), 129-133.

Woon, F. L., \& Hedges, D. W. (2008). Hippocampal and amygdala volumes in children and adults with childhood maltreatment-related posttraumatic stress disorder: a meta-analysis. Hippocampus, 18(8), 729-736.

World-Bank. (2001). Engendered development. New York: Oxford University Press.

Wright, R. O., Silverman, E. K., Schwartz, J., Tsaih, S. W., Senter, J., Sparrow, D., Weiss, S. T., Aro, A., \& Hu, H. (2004). Association between hemochromatosis genotype and lead exposure among elderly men: the normative aging study. Environmental Health Perspectives, 112(6), 746-750.

Wu, T., Snieder, H., \& de Geus, E. (2009). Genetic influences on cardiovascular stress reactivity. Neuroscience and Biobehavioral Reviews.

Xiong, G. L., Plassman, B. L., Helms, M. J., \& Steffens, D. C. (2006). Vascular risk factors and cognitive decline among elderly male twins. Neurology, 67(9), 1586-1591.

Xu, G., Zhou, Z., Zhu, W., Fan, X., \& Liu, X. (2009). Plasma C-reactive protein is related to cognitive deterioration and dementia in patients with mild cognitive impairment. Journal of Neurological Sciences, 284(1-2), 77-80. 
Yaffe, K., Haan, M., Blackwell, T., Cherkasova, E., Whitmer, R. A., \& West, N. (2007). Metabolic syndrome and cognitive decline in elderly Latinos: findings from the Sacramento Area Latino Study of Aging study. Journal of the American Geriatric Society, $55(5), 758-762$.

Yaffe, K., Kanaya, A., Lindquist, K., Simonsick, E. M., Harris, T., Shorr, R. I., Tylavsky, F. A., \& Newman, A. B. (2004). The metabolic syndrome, inflammation, and risk of cognitive decline. Journal of the American Medical Association, 292(18), 2237-2242.

Yaffe, K., Lindquist, K., Penninx, B. W., Simonsick, E. M., Pahor, M., Kritchevsky, S., Launer, L., Kuller, L., Rubin, S., \& Harris, T. (2003). Inflammatory markers and cognition in wellfunctioning African-American and white elders. Neurology, 61(1), 76-80.

Yaffe, K., Weston, A. L., Blackwell, T., \& Krueger, K. A. (2009). The metabolic syndrome and development of cognitive impairment among older women. Archives of Neurology, 66(3), 324-328.

Yakovlev, P. L. (1967). Regional Development of the Brain in Early Life. Oxford: Blackwell.

Yang, Y. K., Nelson, L., Kamaraju, L., Wilson, W., \& McEvoy, J. P. (2002). Nicotine decreases bradykinesia-rigidity in haloperidol-treated patients with schizophrenia. Neuropsychopharmacology, 27(4), 684-686. 
Yavich, L., Forsberg, M. M., Karayiorgou, M., Gogos, J. A., \& Mannisto, P. T. (2007). Sitespecific role of catechol-O-methyltransferase in dopamine overflow within prefrontal cortex and dorsal striatum. Journal of Neuroscience, 27(38), 10196-10209.

Yehuda, R., Golier, J. A., \& Kaufman, S. (2005). Circadian rhythm of salivary cortisol in Holocaust survivors with and without PTSD. American Journal of Psychiatry, 162(5), 9981000.

Yilmaz, N., Herken, H., Cicek, H. K., Celik, A., Yurekli, M., \& Akyol, O. (2007). Increased levels of nitric oxide, cortisol and adrenomedullin in patients with chronic schizophrenia. Medical Principles and Practice, 16(2), 137-141.

Young, E. A. (1998). Sex differences and the HPA axis: implications for psychiatric disease. Journal of Gender-Specific Medicine, 1(1), 21-27.

Yucel, K., McKinnon, M. C., Taylor, V. H., Macdonald, K., Alda, M., Young, L. T., \& MacQueen, G. M. (2007). Bilateral hippocampal volume increases after long-term lithium treatment in patients with bipolar disorder: a longitudinal MRI study. Psychopharmacology (Berl), 195(3), 357-367.

Zala, S. M., \& Penn, D. J. (2004). Abnormal behaviours induced by chemical pollution: a review of the evidence and new challenges. Animal Behaviour, 68, 649-664. 
Zhang, J., Vitaliano, P. P., \& Lin, H. H. (2006). Relations of caregiving stress and health depend on the health indicators used and gender. International Journal of Behavioral Medicine, $13(2), 173-181$.

Zhang, X. Y., Zhou, D. F., Cao, L. Y., Wu, G. Y., \& Shen, Y. C. (2005). Cortisol and cytokines in chronic and treatment-resistant patients with schizophrenia: association with psychopathology and response to antipsychotics. Neuropsychopharmacology, 30(8), 15321538.

Zhang, X. Y., Zhou, D. F., Qi, L. Y., Chen, S., Cao, L. Y., Chen da, C., Xiu, M. H., Wang, F., Wu, G. Y., Lu, L., Kosten, T. A., \& Kosten, T. R. (2009). Superoxide dismutase and cytokines in chronic patients with schizophrenia: association with psychopathology and response to antipsychotics. Psychopharmacology (Berlin), 204(1), 177-184.

Zimmer, K. E., Gutleb, A. C., Lyche, J. L., Dahl, E., Oskam, I. C., Krogenaes, A., Skaare, J. U., \& Ropstad, E. (2009). Altered stress-induced cortisol levels in goats exposed to polychlorinated biphenyls (PCB 126 and PCB 153) during fetal and postnatal development. Journal of Toxicology and Environmental Health, 72(3-4), 164-172.

Zimmermann, U. S., Blomeyer, D., Laucht, M., \& Mann, K. F. (2007). How gene-stress-behavior interactions can promote adolescent alcohol use: the roles of predrinking allostatic load and childhood behavior disorders. Pharmacology, Biochemistry, and Behavior, 86(2), 246262. 
Zitzmann, M., \& Nieschlag, E. (2007). Androgen receptor gene CAG repeat length and body mass index modulate the safety of long-term intramuscular testosterone undecanoate therapy in hypogonadal men. Journal of Clinical Endocrinology and Metabolism, 92(10), 3844-3853.

Zou, K., Maeda, T., Watanabe, A., Liu, J., Liu, S., Oba, R., Satoh, Y., Komano, H., \& Michikawa, M. (2009). Abeta42-to-Abeta40- and angiotensin-converting activities in different domains of angiotensin-converting enzyme. Journal of Biological Chemistry, 284(46), 3191431920.

Zubieta, J. K., Heitzeg, M. M., Smith, Y. R., Bueller, J. A., Xu, K., Xu, Y., Koeppe, R. A., Stohler, C. S., \& Goldman, D. (2003). COMT val158met genotype affects mu-opioid neurotransmitter responses to a pain stressor. Science, 299(5610), 1240-1243.

Zuch, C. L., O'Mara, D. J., \& Cory-Slechta, D. A. (1998). Low-level lead exposure selectively enhances dopamine overflow in nucleus accumbens: an in vivo electrochemistry time course assessment. Toxicology and Applied Pharmacology, 150(1), 174-185. 